\title{
PHOTOIONIZATION STUDIES WITH MOLECULAR BEAMS
}

\author{
Cheuk-Yiu Ng
}

Materials and Molecular Research Division, Lawrence Berkeley Laboratory Department of Chemistry; University of California Berkeley, Cailifornia 94720

\section{ABSTRACT}

A molecular beam photoionization apparatus which combines the advantages of both the molecular beam method with photoionization mass spectrometry has been designed and constructed for carrying out some unique photoionization experiments. It essentially consists of a differentially pumped windowless vacuum ultravioiet monochromator, a modulated supersonic beam production system, an ionization chamber, phaton detectors, a low noise quadrupole mass spectrometer and intense continuum light sources (extending from $600 \AA$ to $2000 \AA$ ).

Rotational cooling during the supersonic expansion has resulted in high resolution photoionization efficiency curves for NO, ICI, $\mathrm{C}_{2} \mathrm{H}_{2}$ and $\mathrm{CH}_{3} \mathrm{I}$. The analysis of these spectra has yielded ionization potentials for these molecules to an zccuracy of \pm 3 meV. Detailed autoionization structures were also resolved. This allows the investigation of the selection rules for autolonization, and the identification of the Rydberg series which converge to the excited states of the molecular ions. The degree of relaxation for thermally populated excited states has been examined using $\mathrm{NO}$ and ICl as examples. As a result of adiabatic cooling, a small percentage of dimers is also formed during the expansion. The photoionization efficiency curves for 
$\left(\mathrm{NO}_{2}, \mathrm{ArICl}, \mathrm{Ar}_{2}, \mathrm{Kr}_{2}\right.$ and $\mathrm{Xe}_{2}$ have been obtained near the thresholds. Using the known dissociation energies of the $(\mathrm{NO})_{2}, \mathrm{Ar}_{2}, \mathrm{Kr}_{2}$ and $\mathrm{Xe}_{2}$ van der Waals molecules, the corresponding dissociation energies for $\mathrm{NO}-\mathrm{NO}^{+}, \mathrm{Ar}_{2}^{+}, \mathrm{Kr}_{2}^{+}$, and $\mathrm{Xe}_{2}^{+}$have been determined. The ionization mechanisms for this class of molecules is examined and discussed. 


\section{ACKNOWLEDGHENTS}

I would like to express my deepest appreciation to Professor Y. T. Lee for his support, understanding, and assistance throughout the course of this research. His physical instght and encouragenent have made my graduate studies to be one of the most exciting and fruttful periods in my life. Special acknowiedgement goes to Professor B. H. Mahan for his advice and helpful suggestions during the last two years. I would also like to thank my many colleagues, Dr. T. P. Schaefer, Dr. F. P. Tully, Dr. J. M. Farrar, Or. C. H. Chen, Dr. K. Shobatake, Mr. J. J. Valentini, Dr. M. J. Coggiola, Mr. R. K. Sparks, Mr. P. A. Schulz, Mr. 2. F. Hang, Mr. C. J. Becker, Mr. R. J. Buss, Mr. D. J. Trevar, Mr. S. J. Sibener, Mrs. C. C. Kahler, Miss P. L. Kronebusch and Mr. R. C. Sharp for their assistance, friendship and enitghtening discussion.

The assistance of Or. J. Conway and Mr. G. Shalinoff in aligning the monochromator and lending us the discharge lamp power supply is greatly apprectated. Special acknowledgement extends to Professor W. A. Chupka for his helpful advice in the design and construction of the vacuum ul traviolet light source, and Mr. G. Gabor for helpful suggestions in the design of the scinthllation detector. I wish to take this opportunity to acknowledge the skillful issistance of the technical staff of the machine, ceramic, glass and electronic shop of the Lawrence Berkeley Laboratory during the development of the instruments, and to thank Mrs. Ann Heightntan for cyping the manuscript. 
I an most grateful to my wife for her love, and deep understanding and encouragement. 
TABLE OF CONTENTS

Chapter One:

Chapter Two:

2.1

$$
2.2
$$

Chapter Three:

Chapter Four:

Chapter Five:

Chapter Six:

Chapter Seven:

Chopter Eight:
Introduction

Theoretical Considerations

6

Direct Ionization

6

Autoionization

Photoionization with Molecular Beams

1. Autolonization Structure of Mitric Oxide 16 Mear the Threshold

The Binding Energy Between $\mathrm{NO}$ and $\mathrm{MO}^{+}$

Photoionization Studies of the $\mathrm{Xe}_{2}, \mathrm{Kr}_{2}$ and $\mathrm{Ar}_{2}$
van der Waals Molecules

Photoionization Study of ICl and the ArICI van der Haals Molecules

Photoionization Study of Acetylene $\left(\mathrm{C}_{2} \mathrm{H}_{2}\right)$ and Methyl Iodide $\left(\mathrm{CH}_{3} \mathrm{I}\right)$

Improved Potentials for $\mathrm{Ne}+\mathrm{Ar}$, Ne $+\mathrm{Kr}$ and $\mathrm{Ne}+\mathrm{Ke}$

Appendices

Section A:

Mechanical and Vacuum Layout of the Photoionization Apparatus

139

Section 8:

Molecular Beam Production System

144

Section C:

Vacuum Ul traviolet Light Source

149

Section D:

Quadrupole Mass Spectrometer

160

Section E:

The Vacuum U1 travinlet Photon Detectors

170

Section $\mathbf{F}$

Automatic Scan Control System for Photoionization 
I. INTRODUCTION

The major processes resulting from the absorption of a vacuum photon by an atom or a molecule may be summarized as follows:
(1) $x+h v+x$
Atomic Excitation
(2) $x+h v+x^{+}+e+K . E$.
Atomic Phototonization
(3)
$x+h v \rightarrow x^{\star} \rightarrow x^{+}+e+k . E$.
Atomic Autoionization
(4) $X Y+h v+X Y^{\star}$
Molecular Excitation
(5) $X Y+h v+X+Y+$ K.E.
$(X$ and/or $Y$ can be
Molecular Dissociation an excited state)
(6) $X Y+h v+X Y^{+}+e+K . E$.
Molecular Photoionization

$$
X Y+h v+X Y^{*}+X+Y+K . E \text {. }
$$
Predissociation
(8) $X Y+h v \rightarrow X y^{\star}+X y^{+}+e+K . E$.
Molecular Autoionization
(9) $x y+h v-x y^{+}+e+x^{+}+y+e$
Fragmentation of Parent Ion
(10) $X y+h v+X y^{*}+X^{+}+y^{-}$
Ion-Pair Formation

The study of these processes, which provide detailed information on the electronic state structures of gases, atoms and molecules as well as atomic and molecular ions, is a fundamental subject of physics and chemistry. The mechanism of UV photon interactions with gases is very important to the understanding of the events associated with phenomena ranging from electrical discharges and plasma physics, upper atmospheric aeronomy and astrophysics. Nevertheless, it is apparent that no single type of experiment is able to carry out detailed Investigation of all these processes. As a matter of fact, it is only from different complementary experimental techniques that a full 
picture of these mechanisms can be realized.

In vacuum ultraviolet absorption spectroscopy, the total absorption cross section is liteasured as a function of photon energy. This method provides the most accurate means of detemining ionization potentials in the case where analysis of the spectrum allows identification of a sufficient number of Rydberg levels. For atoms, the absorption crosssection will closely approximate the ionization cross section, whereas for molecules other processes such as dissociation may occur. It is often true that due to the broadening of absorption lines because of predissociation, etc., that the identification of the Rydberg series is made difficult or impossible.

Photoelectron spectroscupy involves the measurement of the kinetic energies of the ejected electrons by the interactions of a monoenergetic beam of photons with an atom or a molecule. This method is an especially unambiguous one for the study of molecular electronic structures of substances in a vapor state. However, the lack of mass discrimination to assist in the identification of the ions formed often makes the analysis of the spectra very difficult.

The measurement of the ionization cross section for a particuiar ionizing channel as a function of photon energy is the object of the photoionization experiment. The photoionization efficiency curve (number of Ions produced per photon transmitted, plotted as a function of wavelength) will, if taken at sufficient high resolution, usually contains information on the first and higher ionization potentials, vibrational frequency of the ion, and Franck-Condon factors for the 
ionization process. Also found in the photoionization efficiency curves are sharp peaks due to autoionization, in which the lonization takes place in two stages. The first step is a resonant process involving the excitation of the neutral species to a discrete rescnant state above its fonization potentials, and the second is the spontaneous loss of the electron. This structure allows the investigation of the coupling between discrete states and ionization continuum (i.e., the selection rules and lifetime etc. for autoionization). Furthemore. the measurement of ionization and appearance potentials can, by co:sideration of suitable thermodynamic cycles, provide a great deal of thermochemical data for both ionic and neutral species. Consider the case of molecular photoionization in process (6). The appearance potential is

$$
\operatorname{AP}\left(X Y^{+}\right)=\Delta H_{f}\left(X Y^{+}\right)-\Delta H_{f}(X Y)
$$

Since $\Delta H_{f}(X Y)$ is known for a large number of compounds, then $\Delta H_{f}\left(X Y^{+}\right)$ can be determined. Another example as in process (9) we have

$$
\operatorname{AP}_{g}\left(X^{+}\right)=\operatorname{IP}(X)+D(X-Y)
$$

where either the bond dissociation energy may be known from other experiments or it may be possible to measure the ionization potential of $X$ from the above equation.

If the ton pair process also occurs, the difference in the appearance potential for process $(10), \operatorname{AP}_{10}\left(X^{+}\right)$, and that of process (9), $\operatorname{AP}_{9}\left(X^{+}\right)$give the electron affinity of $Y$. That is

$$
\operatorname{AP}_{9}\left(X^{+}\right)-\operatorname{AP}_{10}\left(X^{+}\right)=E A(Y)
$$


However, as mentioned above, the full potential of the photoionization experiment will be fully realized only if high resolutis photoionization efficiency curves are obtained. At the present technological level, a windowless vacuum ultraviolet monochromator operated by extensive differentlal pumping and an intense laboratory continuum vacuum ultraviolet light source are available. These enable the usual instrumental resolution for photoionization experiments to be as high as 1-10 meV without suffering from very low ion intensity. However, it is the rotational envelope of the molecules at room temperat: re that limits the ultimate resolution. For diatomic molecules, the rotational envelope half-width within a single electronic vibration is $n f$ the order of $\mathrm{kT}=0.025 \mathrm{eV}$, while that of polyatomic molecule is about $39 \mathrm{meV}$. The rotational spread can be suppressed by cooling the gas to low temperature. In the case of hydrogen, by cooling the gas to liquid nitrogen temperature, an energy resolution as high as 0.04 .4 has been obtained by Chupka and co-workers. ' Obviously, this is not applicable to condensable gases. One way to circumvent this difficulty is to use the modulated supersonic beam technique. It is known that adiabatic cooling of the gas during the expansion is extremely effective in lowering the rotational temperature. Furthermore, the beam technique also allows the photoionization of dimers and transient radicals to be possible. This thesis reports the results of high resolution photoionization studies of condensable gases ( $\mathrm{NO}, \mathrm{ICl}, \mathrm{CH}_{3} \mathrm{I}$ and $\mathrm{C}_{2} \mathrm{H}_{2}$ ) and dimers $\left(\left(\mathrm{NO}_{2}, \mathrm{Xe}_{2}, \mathrm{Kr}_{2}\right.\right.$ and $\left.\mathrm{Ar} \mathrm{r}_{2}\right)$ using the molecular beam method. 
$-5-$

\section{REFERENCES}

1. W. A. Chupka and s. Berkowitz, J. Chesi. Phys., 51, 4244 (1969). 


\section{Theoretical Considerations}

The ionization may be a direction process which involves a direct transition of a bound electron into the ionization continuum, or may take place by an indirect process, for example autoionization and predissociation.

\subsection{Direci Ionization}

It has beeñ shown both theoretically $y^{1,2}$ and experimentally ${ }^{3}$ that the threshold for photoionization is a step-like function, i.e., the onset rises sharply at the threshold of each process and then tends to assume a constant value. To a first approximation, the cross section for photoionization can be calculated by considering the interaction of a photon with an atomic or molecular system to be analogous to the interaction of an electromagnetic wave with an electric dipole. If the wavelength of the incoming photon is long compared with the distance over which the wavefunctions are appreciable, the dipole approximation is satisfactory, and the operator describing the interaction of two wavefunctions may be taken to be the dipole operator. 4 Assuming only a single electron to be involved in the transition, the expression for total photoionization cross section is 5

$$
\sigma_{v}=\frac{4 \pi^{2} \alpha a_{0}^{2}}{3} \frac{v_{i}+k^{2}}{g_{i}}\left|M_{i f}\right|^{2}
$$

$M_{\text {If }}$ is the matrix element defined by

$$
\left|M_{i f}\right|^{2}=\sum_{i} \sum_{j}\left|\rho \Psi_{f}^{*} \sum_{\mu} r_{\mu} \Psi_{i} d c\right|^{2}
$$

$\sigma_{v}$ is the photoionization cross section at some radiation frequency $v$. 
$m$, e, are the mass and charge respectively of an electron.

$\mathbf{g}_{\mathbf{j}}$ is the degeneracy of the initial state.

$\sum_{i} \sum_{j}$ denote sums over the initial and final state respectively.

$\Psi_{j}$ is the initial state wavefunction.

$\psi_{f}^{\star}$ is the final state wavefunction.

$k^{2}$ is the kinetic energy of the ejected electron.

$v_{i}$ is the ionization threshold energy.

$\sum_{\mu} r_{\mu}$ characterizes the dipole moment operator.

$\alpha$ is the fine structure constant.

$a_{0}$ is the Bohr radius.

Equation (1) indicated that the cross-section depends not only on the matrix element $M_{i f}$ but also on the frequency of the incident radiation and the velocity of the ejected electron. Let us consider the photoionization partial cross section which only involves the transition from $\Psi_{i}$ to $\psi_{f}$ and let $r$ and $R$ denote the electronic and nuclear coordinates respectively. Assuming the photoionization process is rapid with respect to the vibrational period, 1.e. the molecule contains a fixed nuclear geometry during this process, we can introduce the Born-Oppenheimer approximation and hence $\Psi_{i, f}$ can be written as the product of an electronic and a vibrational wavefunction (neglecting rotation) i.e., $\Psi_{i}=\psi_{e} " \psi_{v^{\prime \prime}}$ and $\Psi_{f}=\psi_{e} \cdot \psi_{v^{\prime}}$. The transition probability

$$
P_{v^{\prime} v^{\prime \prime}} \times \mid \int \psi_{e^{\prime}} \psi_{v^{\prime}}\left(M_{r}+M_{R}\right) \psi_{\left.e^{\prime \prime} \psi_{v^{\prime \prime}} d r d R\right|^{2}}
$$

$M_{r}$ and $M_{R}$ are the dipole moment operators which depends on electronic and nuclear coordinates respectively. If $\Psi_{e^{\prime}}$ and $\psi_{e^{\prime \prime}}$ are orthorgonal 
wavefunctions, we have

$$
\begin{aligned}
& P_{v^{\prime}, v^{\prime \prime}} \times \int \psi_{e} \cdot M_{r} \psi_{e^{\prime \prime}} d r \int \psi_{v^{\prime}} \psi_{v^{\prime \prime}} d R+\left.\int \psi_{e} \cdot \psi_{e^{\prime \prime}} d r \int \psi_{v^{\prime}} M_{R^{\prime}} \psi_{v^{\prime \prime}} d R\right|^{2} \\
& =\left|\int \psi_{e} \cdot M_{r} \psi_{e^{\prime \prime}} d r\right|^{2}\left|\int \psi_{v} \cdot \psi_{v}{ }^{\prime d} d R\right|^{2} \\
& =|\overrightarrow{\mathrm{Pe}}|^{2}\left|\int \psi_{v^{\prime}} \psi_{v^{\prime \prime}} \mathrm{dR}\right|^{2}
\end{aligned}
$$

where $\left|\int \psi_{v^{*}} \psi_{v^{\prime \prime}} d R\right|^{2}$ is the Franck-Condon factor which gives relative strengths of electroric transitions and distributes the intensity into appropriate vibrational states. For states of the ion having equilibrium distance different from that in the ground state of the neutral molecule, maximum wavefunction overlap (Franck-Condon region) occurs at a position on the potential energy surface of the ion different from the ground state. The most commonly observed FranckCondon overlap conditions in photoionization are shown in Fig. (1). The transitions are all taken as originating from the zero vibrational level of the initial electronic state. The maximum probability for a transition occurs in the center of the Franck-Condon region where the initial wavefunctions has its maximum. The three cases shown are: a. The ionization only involves in removal of a nonbinding or "lone pair" electron, which has little effect in the bonding of the molecular ion. In other words, there is little or no change in internuclear distance or vibrational interval from that of the neutral molecule. The photoionization efficiency curve will essentially show one big step corresponding to an intense 0-0 transition as indicated in curve (a) of Fig. 1. 
$-9-$

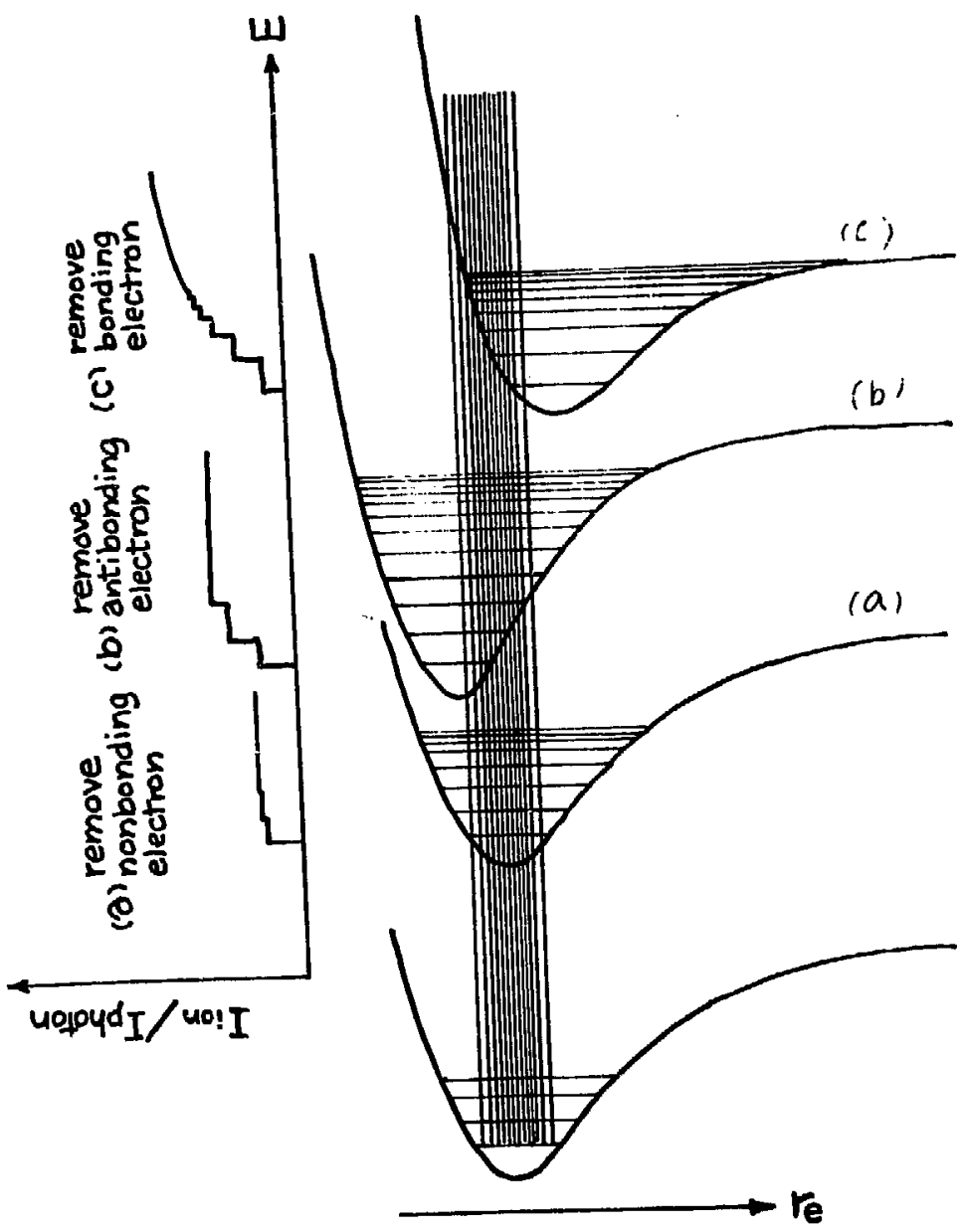

Fig. 1 Application of Franck-Condon Principle to photoion Production 
b. The removal of an antibonding electron results in strengthening the bonding of the molecular ion. This implies a decrease in bond length and wider vibrational spacing for the ionic state. The photoionization efficiency curve hence will show a few widely spaced steps as shown in curve (b) of Fig. 1 (e.g. No).

c. Removal of a bonding electron produces transitions to the steeper slope of the strongly repulsive portion of the ion potential well. The photoionization efficiency curve usually is composed of a closely spaced steps indicating an increase in bond length (weaker bonding) and frequency reduction in the molecular ion with respect to that in the neutral molecule. (e.g. $\left.\mathrm{C}_{2} \mathrm{H}_{2}\right)$. This is shown in curve (c) of Fig. 1.

If $|\overline{\mathrm{Pe}}|^{2}$ is assumed independent of total energy and photoelectron energy, the intensity ratio corresponding to the transition from the ground state $\Psi_{v^{\prime \prime}}$ to two vibrational states $\left(v^{\prime}+1, v^{\prime}\right)$ of the molecular ion will bear the relation

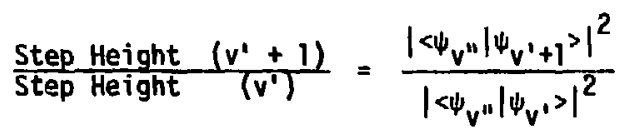

\subsection{Autoionization}

The removal of an electron from an atom or molecule which does not involve a transition directly into the continuum is known as autolonization. This process arises due to the overlipping of discrete energy levels by a continuous range of levels as depicted in Fig. 2 . Series 1 represents the Rydberg levels converging to the first fonization potential and series 2 the Rydberg levels leading to a 


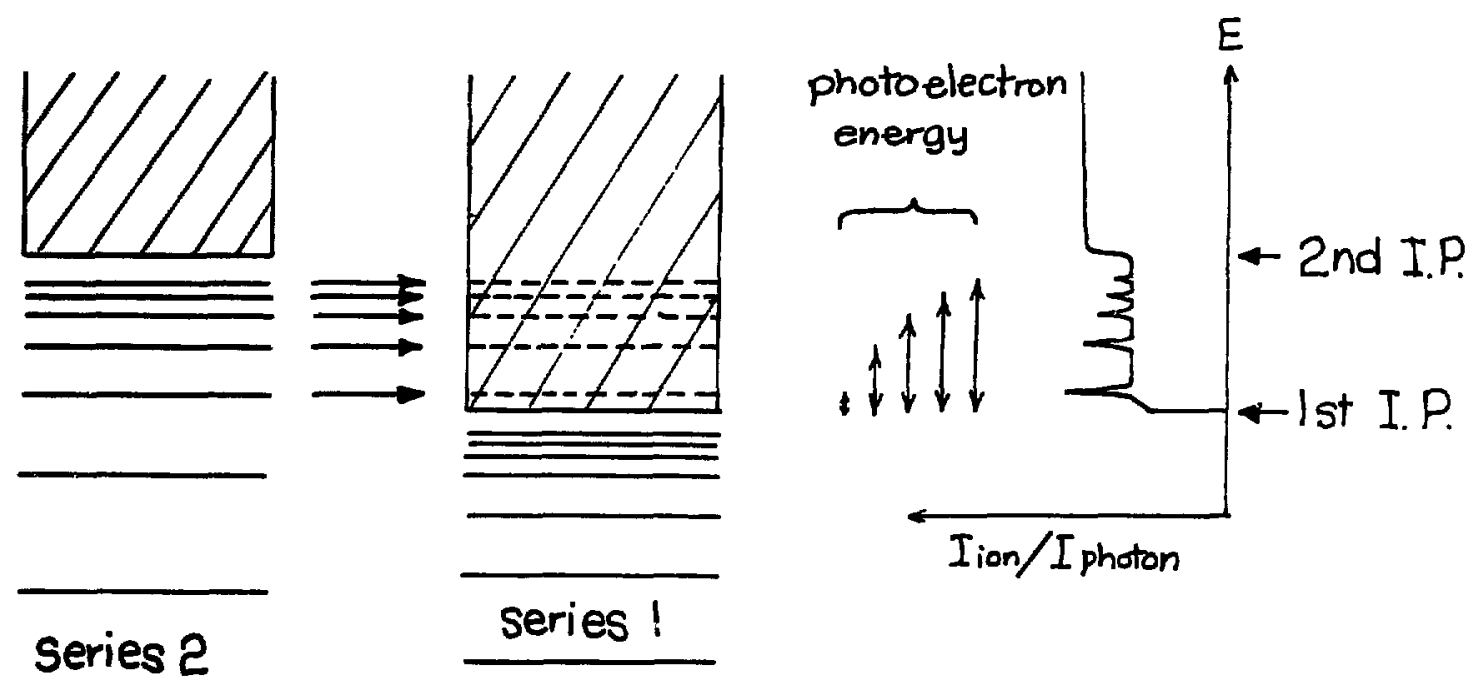

neutral

ground siate

Fig. 2. Autoionization of atoms \& Molecules after excitation Levels above the 1st. I.P. 
higher fonic state. If an electron is excited to a bound level in series 2 which is higher in energy than the first ionization potential associated with series 1 , then the possibility exists of a radiationless transition from the bound molecular state into a stable fonil: state. The electron ejected in this manner will have a kinetic energy equal to the difference between the energy of the bound state and the lower ionization potential. Unlike the direct ionization, autoionization is a resonant process occurring only at discrete energies which correspond to the bound levels of series 2 . The process is manifested as a number of peaks on the photoionization efficiency curve as shown in Fig. 2.

The phenomenon was first postulated by Shenstone ${ }^{7}$ to explain the broadening and asymmetry of some absorption peaks above the Ionization potential of certain species. The theoretical aspects of autoionization have been investigated extensively. Fano ${ }^{8}$ postulated configuration interaction between the discrete states and the continuum, and has studied theoretically the asymetric peaks of autoionization which appear in the photoionization efficiency curve. He has been able to predict the position and intensity shifts produced in a Rydberg series due to autoionization. Mies ${ }^{9}$ has extended Fano's theory of configuration interaction to include the case of many discrete states interacting with many continuum channels, with the possibility that neighboring resonances may overlap, i.e., that their autoionization widths may be greater than the spacing between discrete states. The transition amplitude is 


$$
\hat{t}_{v^{\prime}}^{-}=\sum_{\alpha} F_{v^{\prime}, \alpha^{F}} F_{\alpha, v^{\prime \prime}} C_{\alpha}
$$

where $c_{\alpha}=t_{e}\left[1+q \sum_{n^{*}} \varepsilon_{\alpha, n^{*}}^{-1}\right] /\left[1+1 \sum_{n^{*}} \varepsilon_{\alpha, n^{*}}^{-1}\right]$

$$
\begin{aligned}
\varepsilon_{\alpha, n^{*}} & =\left(\varepsilon-\varepsilon_{\alpha, n^{*}}\right) /(1 / 2 \Gamma) \\
\Gamma & =\sum_{v^{\prime}} \Gamma_{v^{\prime}}=2 \sum_{v^{\prime}}\left|<\Psi_{v^{\prime}, f^{\prime}}\right| H\left|\Psi_{\alpha, n^{*}}\right|^{2}
\end{aligned}
$$

$t_{v^{\prime}}^{-}$is the transition amplitude from ground vibrational state $v^{\prime \prime}$ to final ionic vibrational state $v^{\prime}$.

$n * \& \alpha$ are the effective principle quantum number and vibrational quantum number respectively which characterize the Rydberg state. $q$ is the Fano's line profile index.

$v^{\prime \prime}, \alpha, v^{\prime}$ are the index for denoting the vibrational quantum number for the ground state, Rydberg state and final molecular fonic state. $F_{v^{\prime}, \alpha} \& F_{\alpha, v^{\prime \prime}}$ are the Franck-Condon factors between the corresponding vibrational levels.

$H$ is the interelectronic repulsion causing the two electron transition. $\Gamma$ is the total autoionization width of state $\left(n^{*}, \alpha\right)$. $t_{e}$ is a factor which depends on the electronic transition probability. $E$ is the incident photon energy.

If the widths of the states are much less than the separation between successive states, autoionization will be important only if : is close to the energy of some state $n^{\star}, \alpha$, then

$$
c_{\alpha}=t_{e}\left[\varepsilon_{\alpha, n^{\star}}+q\right] /\left[\varepsilon_{\alpha, n^{*}}+i\right]
$$

which is Bardsley's result. ${ }^{10}$ If $\varepsilon$ is far from any sharp resonance $c_{\alpha}=t^{c}$ for all $\alpha$ 


$$
t_{v^{\prime}}^{-}=t_{e} \sum_{\alpha} F_{v^{\prime} \alpha^{\prime} \alpha, v^{\prime \prime}}=t_{e} F_{v^{\prime}, v^{\prime \prime}}
$$

which is the direct phototonization result. However if $\varepsilon$ falls precisely on a single resonance $\left(n^{\star}, \alpha\right)$ which does not overlap with any other resonance, then

$$
t_{v^{\prime}}^{-}=t_{e}\left[F_{v^{\prime \prime} v^{\prime}}-F_{v^{\prime \prime} \alpha^{\prime} \sigma_{v^{\prime}}}(1+i q)\right]
$$

The first term in bracket is the contribution of direct photoionization and the second is the contribution of the autoionizing state. If the second term predominates, the intensity ratio of two vibrational peaks $\left(v^{\prime}+1, v^{\prime}\right)$ now has the form ${ }^{11}$

$$
\frac{\text { intensity }\left(v^{\prime}+1\right)}{\text { intensity } v^{\prime}}=\left(\frac{F_{v^{\prime}+1, \alpha}}{F_{v^{\prime} \alpha}}\right)^{2}
$$




\section{REFERENCES}

1. R. W. Ditchburn, U. Opik, "Atomic and Molecular Processes" ed.

D. R. Bates, Academic Press, London (1962).

2. S. Geltman, Phys. Rev., 112, 176 (1958).

3. J. A. R. Samson, J. Op. Soc. Am., 55, 935 (1965).

4. E. W. McDaniel, "Collision Phenomena in Ionized Gases." Wiley, New York (1964).

5. D. R. Bates, Mon. Not. Roy. Astron. Soc., 106, 423 (1946).

6. D. A. S. Vroom, Ph.D. Thesis, The University of British Columbia (1966).

7. A. G. Shenstone, Phys. Rev., 38, 873 (1931).

8. U. Fano, Phys. Rev., 124, 1866 (1961).

9. F. H. Mies, Phys. Rev., 175, 164 (1968).

10. J. N. Bardsley, Chem. Phys. Lett., 2, 329 (1968).

11. A. L. Smith, Phil. Trans. Roy. Soc. Lond., A 268, 169 (1970). 
III. PHOTOIONIZATION WITH MOLECULAR BEAMS

I. AUTOIONIZATION STRUCTURE OF NITRIC OXIDE NEAR THE THRESHOLD

\section{ABSTRACT}

The phototonization effictency curve of $\mathrm{NO}^{+}\left(\mathrm{X}^{1} \Sigma^{+}\right)$was obtained using a molecular beam technique in the wavelength range from $1350 \AA$ to $1185 \AA$ (9.18-10.46 eV). Due to the lowering of the rotational temperature by supersonic expansion, the autoionization structure superimposed on the vibrational steps is well resolved. This structure is attributed to vibrational autoionization. The splitting of the autoionization peaks due to the spin-orbit ground state $\left({ }^{2} \pi_{1 / 2},{ }^{2} \pi_{3 / 2}\right)$ of nitric oxide is observed. The strong intensity peaks of the vibrational autoionization are found to correspond to a vibrational quantum number change of $\Delta v=-1$. The autoionization structure observed on the first four vibrational steps obeys the propensity rule proposed by Berry. 1 
INTRODUCTION

Since advances in vacuum technology and improvements of diffraction gratings have extended high resolution molecular spectroscopy into the vacuum ultraviolet region, many new electronic states and Rydberg series of molecules and atoms have been identified in absorption and emission. However, the absorption of ultraviolet radiation by molecules is observed to be considerably more complex than that of atoms. When the overlapping of discrete energy levels by a continuous range of levels occurs, both autoionization and predissociation can take place and reduce the lifetime of the discrete state, with concomitant broadening of the absorption features. The high resolution photoionization experiment renains one of the most sensitive methods of investigating the ionization processes and coupling mechanisms between the excited Rydberg states and ionic states. Not only does it give precise information on the first and higher ionization potentials of molecules and vibrational frequencies of the ions, but Franck-Condon factors for the direct ionization can also be obtained from the relative height of the steps which appear in the photoion yield curves. The most complete system of Rydberg states for a diatomic molecule other than $\mathrm{H}_{2}$ and $\mathrm{He}_{2}$ has emerged in recent years from the study of high resolution emission and absorption spectra of NO, which has been principally carried out by Miescher and his co-workers. ${ }^{1-5}$ In a conventional absorption spectroscopic experiment, detection of weak autoionization structure can be difficult. These difficulties are lessened to a great extent in a high resolution photoionization experiment, where the lans are directly detected. 
The photoionization of NO has been studied by many investigators in the last 20 years. ${ }^{6-12}$ Step function behavior of the photoionization efficiency curve has been demonstrated and the fine structure superimposed upon the steps has also been observed. ${ }^{11,12}$ A more detailed investigation of the threshold region was undertaken by Killgoar et. al. 13 only recently. Their experiment was done with a dispersed vacuum ulträviolet light on a gas cell of nitric oxide cooled to $150^{\circ} \mathrm{K}$. The autotonizing peaks observed in their work are still relatively broad. Hence, the assignment of the autoionization structure might not be entirely certain. Even though Killgoar et. al. used a dispersed photon beam of about 6 meV resolution, the resolution of their experiment was actually limited by the rotational temperature of $\mathrm{NO}$ at $150^{\circ} \mathrm{K}(\sim 13 \mathrm{meV})$. In order to take full advantage of the energy resolution available by photoionization experiments, the gas cell has to be cooled at least down to $80^{\circ} \mathrm{K}$. This is obviously impossible for condensable gases such as NO. One of the most effective methods of surmounting these problems is to employ the supersonic beam technique. We have used this method to obtain the photoionization efficiency curve of NO with our molecular beam photoionization apparatus. The autoionization structure observed is re-interpreted as discussed below.

\section{EXPERIMENTAL}

The apparatus used in this work is essentially a normal incidence type vacuum-ultraviolet monochromator (McPherson 225) coupled to a molecular beam chamber as shown in Fig. 1 . The photon source is the 
many-line pseudo-continuum of $\mathrm{H}_{2}$ produced by a D.C. capillary discharge. The discharge lamp is made out of quartz, with a $6 \mathrm{~mm} .0 . D . \times 4 \mathrm{~mm}$. I.D. discharge capillary surrounded by a 1" 0.D. standard wall water jacket. The whole discharge lamp is 11 " long. Both the anode and cathode are made out of aluminum and water cooled during the operation of the lamp. Before the main chamber of the monochromator, the source is differentially pumped by a Roots blower and an ejector pump. With the lamp operated at about 5 torr, and with the entrance slit about. $100 \mu$, the monochromator can maintain a vacuum at $5 \times 10^{-6}$ torr. The intensity of the dispersed light coming out from the exit slit is monitored by a sodium salicylate coated photomultiplier, which is known to have a constant quantum efficiency from $300 \AA$ to $1600 \AA{ }^{14}$ (The current output from the photomultiplier is usually about $10^{-7}$ ampere, which is two orders of magnitude higher than the photomultipl ier dark current.) The signal from the photomultiplier is measured by a picoammeter. The stability of the discharge lamp is further monitored by taking the analog output of the picoamuneter to a strip chart recorder. The grating used in the work is $\mathrm{MgF}_{2}$ coated, ruled with 1200 lines/mm and blazed at 1200R. The reciprocal dispersion is $8.3 \AA \mathrm{A} / \mathrm{mm}$, with $100 \mu$ exit and entrance slits, the resolution is about $6 \mathrm{meV}$. Wavelength calibration was accomplished by using the known emission lines of the hydrogen atom and molecule.

The nitric oxide target gas is introduced in the chamber by supersonic expansion through a $0.005^{\prime \prime}$ nozzle with a stagnation pressure of -350 torr. The beam expansion chamber is pumped by a 10" diffusion pump which maintains a vacuum of $10^{-4}$ torr. The second differential pumping region in which the beam is collimated is punped by a 4 " 
diffusion pump which maintains a pressure of $10^{-5}$ torr. The main chamber is pumped by two 4" diffusion pumps, and has a pressure of $5 \times 10^{-7}$ torr when the beam is running. The nitric oxide and photon beams cross in the main chamber at nearly a right angle. The width of both the photon beam and the molecular beam is about $0.3 \mathrm{~cm}$ at the collision region. The detector is a quadrupole mass spectrometer which has its axis perpendicular to both the photon beam and the molecular beam. The whole detector is differentially pumped by a $220 \mathrm{l} / \mathrm{sec}$ ion pump, which provides the detector with a very high vacuum $\left(\sim 5 \times 10^{-9}\right.$ torr $)$ when the main chamber is at $5 \times 10^{-7}$ torr. The ions generated at the collison center are focused into the mass spectrometer and mass analyzed. The molecular beam is chopped by a $150 \mathrm{~Hz}$ tuning fork chopper which generates two gating signals corresponding to the beam on and off. Each gate initiates one of an idential pair of $5 \mathrm{MHz}$ scalers. The difference of the two counters gives the net signal. With the present beam production system, the modulated background is about $5-10 \%$ of the signal counts. The monochroinator is scanned manually using a $0.5 \AA$ interval. At each wavelength, the current reading of the picoammeter which corresponds to the 1 ight intensity is recorded. The relative photoionization efficiency curve is thus equal to the ratio of ion signal to the photon signal. After the experiment, the monochromator is scanned down to $600 \AA$ where there is no light generated by the $\mathrm{H}_{2}$ pseudo-continuum, and the current reading of the phototube corresponds to the background of stray photons from the monochromator. All photon signals are corrected for this background. Depending on the intensity of the light, the minimum counting rate is 
-10 counts/sec and the maximum counting rate is 2000 counts/sec at the

Lyman $a$. The average counting rate at the vibrational steps is -50

counts/sec. The whole spectrum is obtained by counting $100 \mathrm{sec}$ at each point.

The vertical cross section of the apparatus showing the experimental arrangement near the collision center is shown in Fig. 2. The photon beam is coming out from the plane of the figure. An electron bombardment type ionizer is coupled to the collision region to check the beam number density and calibrate the mass spectrometer. With the probe of the ionizer, the beam density in general is about $1.5 \times 10^{12}$ molecules/cc at the collision center.

RESULTS AND DISCUSSION

The photoionization spectrum of NO obtained is shown in Fig. 3. The advantages of combining the photoionization mass spectrometric technique and the molecular beam method are apparent from the sharp features shown in the spectrum. The onset of each vibrational step is extremely sharp. The uncertainty of the rise is less than approximately $0.5 \AA$. In addition to the clear step-function behavior due to the direct ionization process, considerable autoionization structure is well-resolved. The lontzation potentials from the two ground spin-orbit $\left(\pi_{1 / 2}, 3 / 2\right)$ states to the first four vibrational levels of the ionic state $\left(\Sigma^{+}\right)$derived from the middle of the first sharp rise of each vibrational step in the photoionization efficiency curve are listed in Table I. A comparison of the values of the first ionization potential of NO obtained in this and in earlier work is made in Table II. The sharp onsets of the 
photoionization efficiency curve observed in this work correspond to ionization potentials from $\left(x^{2} \Pi_{3 / 2}, v^{\prime \prime}=0\right)$ to the $\left(x^{1} \Sigma^{+}, v^{\prime}=0,1,2,3\right)$ states, and the fonization potentials from the $\left(x^{2} \Pi_{1 / 2}, v^{\prime \prime}=0\right)$ state are just $121 \mathrm{~cm}^{-1}(15 \mathrm{meV})^{15}$ higher. The vibrational spacing is listed and compared the values obtalned previously by other methods in Table III. All of them agree very well.

The onset of the $\left({ }^{1} \Sigma^{+}, v^{\prime}=0\right)$ state has been examined at three different nozzle pressures and the results are shown in Fig. 4. Curves $a, b$, and $c$ correspond to nozzle pressures of 100 torr, 300 torr and 450 torr, respectively. They are essentially indistinguishable from each other; i.e., the rotational temperature of the target gas is already as low as $-80^{\circ} \mathrm{K}$ (the resolution of the photon beam in this work) at a nozzle pressure as low as 100 torr. A 80 torr nozzle beam having a rotational temperature $\leqslant 118^{\circ} \mathrm{K}$ has already been demonstrated by the photoionization work of Parr and Taylor. ${ }^{18}$

One of the most important pieces of information obtained by examining the photoion spectrum concerns the manner in which the excited Rydberg states of molecules couple with the ionization continuum. The only two well-characterized systems are those of $\mathrm{H}_{2}$ and $\mathrm{O}_{2}$. In the case of the hydrogen molecule near threshold, autoionization is purely vibrational induced. The energetics of autoionization involve relaxation of a vibrationally excited core, with the energy being supplied to the Rydberg electron. There have been several theoretical discussions of this problem recently. ${ }^{17-21}$ Although different models have been applied to the process, they all come to the conclusion that the autoionization will be most probable for $\Delta v=-1$, considerably less for $\Delta v=-2$, still less 
for $\Delta v=-3$, etc. This is what Berry ${ }^{22}$ calls the propensity rule. This had been observed earlier in photionization experiments 23,24 and later in absorption spectroscopy. 25

The fact that high resolution photoion spectrum of $\mathrm{H}_{2}$ shows no steps in the apparent continuum at any of the thresholds for the formation of vibrationally excited states of $\mathrm{H}_{2}{ }^{+}$indicates nearly complete autoionization. However, the situation is different for NO, which shows step-function structure. Such structure indicates that direct ionization is the dominant process, while autoionization gives only a weak structure superimposed on the steps. Miescher' has obtained a high resolution photograph of the absorption spectrum in the $1300 \AA$ $1400 \AA$ region and identified several Rydberg series converging to excited vibrational states of the ground $x^{1} \Sigma^{+}$electronic state of the NO ion. Due to the strong absorption background, the identification of the autoionizing lines may not always be feasible, as mentioned above. The energy range of Miescher's work, covers only the first vibrational step $\left(v^{\prime}=0\right)$ of the $X^{1} \Sigma^{+}$state. Killgoar et. al. ${ }^{13}$ extended the work to the 4 th vibrational step $\left(v^{\prime}=3\right)$ by a high resolution photoionization experiment. Their assignment to the autoionization structure mainly follows that of Miescher's. Only a few strong autoionizing peaks were assigned as corresponding to a vibrational energy change $\Delta v=-1$. All others were Identified as converging to highly excited vihr tional states of the ionic $X^{\top} \Sigma^{+}$state. Thus, they do not disprove O' onfirm the propensity rule derived by Berry 17 .22hd Bardsley. 18 $M$ scher has reported that many of the Rydberg series in NO are perturbed. This by no means implies that the predissociation is 
strong enough to obscure the autoionizing structure. On the contrary. as argued in the paper of Killgoar et. a1. ${ }^{13}$ the Rydberg absorptions converging to $v^{\prime}=1$ are expected to be strong.

As can be seen in the photoion yield curve of NO, the autoionization fine structure is relatively weak, yet the peaks are sharp. This permits us to assign the autoionization structure unambiguously. Indeed, the analysis of the spectrum does confirm the strong autoionizatiun structure is favored by the vibrational propensity rule $\Delta v=-1$.

The strong autoionization peaks have been fit with the Rydberg equation $v=T_{v}{ }^{\prime}-\frac{R}{(n-\delta)^{2}}$, where $T_{v}{ }^{\prime}$ is the ionization potential limit to which the corresponding Rydberg transition converges, $R$ is the Rydberg constant, $\delta$ is the quantum defect, $n$ is the principal quantum number, and $v$ is the peak position. By using the ionization potentials obtained (Table I) in this work as the converging 1 imits $T_{v}{ }^{\prime}$, we find that 31 of the strong peaks observed in this work are favored by the vibrational propensity rule $\Delta v=-1$. With a quantum defect $\delta$ of 0.87 , the predicted and observed autoionization peaks for the first four vibrational steps are listed in Table IV A-VII B. The observed autoionization peaks for the first four vibrational steps are listed in Tables IV A-VII B, where they are compared with the predictions of the Rydberg equation in which the quantum defect $\delta$ was 0.87 . Predictions and experiments agree to within $-0.5 \AA$, which is the uncertainty of this work. Essentially, on each vibrational step, there are two major series which are separated by about $15 \mathrm{meV}$. This is exactly the ervergy separation of the two spin-orbit states $l^{2} \pi_{1 / 2}$ and $\boldsymbol{z}_{3 / 2}$ ) of the ground state 10 molecule after a correction for zero point energy is made. The lower energy series of peaks is derived from the ${ }^{2} \Pi_{3 / 2}$ 
state while the other comes from the ${ }^{2} \pi_{1 / 2}$ state. At room temperature, the population of the ${ }^{2} \pi_{3 / 2}$ state is about 56: of that of the ground state ${ }^{2} \pi_{1 / 2}$. Our observations show that a nozzle pressure of $\leq 350$ torr is not effective in reloxing the ${ }^{2} \pi_{3 / 2}$ state of $n_{0}$. The intensities of the doublet peaks will not stand in the ratio of $1: 2$ because the peek of the lower energy member $\left({ }^{2} n_{3 / 2^{-}} x^{1} \Sigma^{*}\right)$ always overlaps the rising edge of

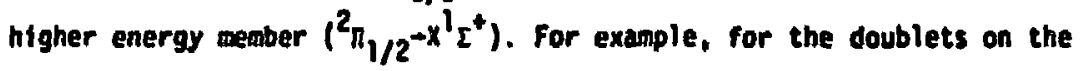
first two vibrational steps, the higher intensity of the $\left(n=8, v^{\prime}=1\right)$ and $\left(n=8, v^{*}=2\right)$ peaks from the ${ }^{2} \pi_{3 / 2}$ state cones from the overlapping of the onset of $\left(n=8, v^{\prime}-1\right)$, and $\left(n=8, v^{\prime}=2\right)$ from ${ }^{2} n_{3 ; 2}$ state respectively.

Table VIII Iists the relative Franck-Condon factor derived in this work and compared with the values obtained by other methods. The accuracy of these values is 1 imited by the autoionizing structure superimposed on the vibrational steps. He adopt the empirical choice of absorption cross-section for direct ionization to be that from base line to autoionization minimum.

In conclusion, we note that our assignments of the autolosization features oiffer in some respects from those of killgoar et. al. ${ }^{13}$ This reassignment is facilitated by the enhanced resolution of our photoionization efficiency curve, which results principally from the rotational cooling achieved by the supersonic expansion. The reassignment has the satisfying aspect that most of the features can be fit with a Rydberg formula in which only one value of the quantum defect is used. 
$-26-$

ACKNOWLEDGMENTS

The authors wish to thank Professor Bradley Moore for providing the vacuum ultraviolet monochromator, and Dr. Conway for lending us the discharge lamp power supply. The helpful discussion with Professor W. A. Chupka on the construction of the vacuum ultraviolet light source is greatly appreciated. This research was supported by the Office of Naval Research and the U. S. Energy Research and Development Administration. 


\section{REFERENCES}

1. E. Miescher, J. Mol. Spectry., 20, 130 (1966).

2. Ch. Jungen and E. Miescher, Can. J. Phys., 47, 1769 (1969).

3. K. P. Huber and E. Miescher, Helv. Phys. Acta., 36, 257 (1963).

4. R. Sutter, Can. J. Phys., 47881 (1969).

5. Ch. Jungen and E. Miescher, Can. J. Phys., 46, 987 (1968).

6. K. Watanabe, F. F. Marmo, and E. C. Y. Inn, Phys. Rev., 91, 1155 (1953).

7. K. Watanabe, J. Chem. Phys., 22, 1564 (1954).

8. H. Hurzeler, M. G. Inghram, and J. D. Morrison, J. Chem. Phys., 28. 76 (1958).

9. A. J. C. Nicholson, J. Chem. Phys., 39, 954 (1963).

10. A. J. C. Nicholson, J. Chem. Phys., 43, 1171 (1965).

11. R. M. Reese and H. M. Rosenstock, J. Chem. Phys., 44, 2007 (1966).

12. K. Watanabe, F. M. Matsunaga and H. Sakai, App1. Opt., 6, 391 (1967).

13. P. C. Killgoar, Jr., G. E. Leroi, J. Berkowitz and W. A. Chupka, J. Chem. Phys., 58, 803 (1973).

14. J. A. R. Samson, J. Opt. Soc. Am., 54, 6 (1964).

15. R. H. Gillette and E. H. Eyster, Physic. Rev., 56, 1113 (1939).

16. G. R. Parr and James W. Taylor, Rev. Sci. Instrum., 44, 1578 (1973).

17. R. S. Berry, J. Chem. Phys., 45, 1228 (1966).

18. J. N. Bardsley, Chem. Phys. Letters, 1, 229 (1967).

19. A. Russek, M. R. Patterson and R. L. Becker, Phys. Rev., 167, 17 (1968).

20. S. E. Nielsen and R. S. Berry, Chem. Phys. Letters, 2, 503 (1968). 
21. B. Ritchie, Phys. Rev., A3, 95 (1971): A6, 1761 (1972).

22. R. S. Berry and S. E. Mielson, Phys, Rev.. Al (1970) 383, 395.

23. W. A. Chupka and J. Berkowitz, J. Chem. Phys., 48, 5726 (1968).

24. J. Berkowltz and H. A. Chupka, J. Chem. Phys., 51, 2341 (1969).

25. G. Honberg and Ch. Jungen, J. Wol. Spectrosc., 4l, 425 (1972).

26. K. P. Huber, Helv. Phys. Acta., 34, 929 (1961).

27. K. Oressler, Astrophys. J., 141, 1266 (1965).

28. K. Watanabe, J. Chem, Phys., 26, 542 (1957).

2S. W. C. Walker and G. L. Weissler, J. Chem. Phys., 23, 1962 (1955).

30. M. I. A1-Joboury, D. P. May, and D. W. Turner, J. Chem. Soc., 616 (1965).

31. D. A. S. Vroom, Ph.D. Thesis, The University of British Columbia (1966).

32. M. I. Al-Joboury and D. W. Turner, J. Chem. Soc., 4434 (1964).

33. P. H. Doolittle and R. L. Schoen, Report No. D1-82-0463, Boeing Scientific Research Laboratories, Seattle (1965).

34. G. G. Cloutier and H. I. Schiff, J. Chem. Phys., 31, 793 (1959).

35. H. Lefebure-Brion and C. M. Mosier, J. Chem. Phys., 44, 2951 (1966).

36. M. E. Wacks, J. Chem. Phys., 41, 930 (1964).

37. E. Miescher, Helv. Phys. Acta., 29, 135 (1956).

38. L. Kerwin, P. Marmet, and E. M. Clarke, "Advances in Mass Spectrometry Vo1. II," Pergamon Press, London (1963).

39. M. Malmann and I. Lanl icht, J. Chem. Phys., 43, 1503 (1965). 
Table I. Ionization Potentials of Nitric Oxide (ev)

(uncertainty $\pm 0.003 \mathrm{ev}$ )

$\overline{\text { Ionic state } \quad\left({ }^{2} \Sigma_{3 / 2}, v^{\prime \prime}=0\right) \rightarrow\left(x^{1} \Sigma^{+}, v^{\prime}\right) \quad\left({ }^{2} \Sigma_{1 / 2}, v^{\prime \prime}=0\right)-\left(x^{1} \Sigma^{+}, v^{\prime}\right)}$

$x^{1} \Sigma^{+}, v^{\prime}=0$

9.256

9.271

$X^{1} \Sigma^{+}, Y^{\prime}=1$

9.547

9.562

$x^{\top} \Sigma^{+}, v^{\prime}=2$

9.834

9.849

$x^{1} \Sigma^{+}, v^{1}=3$

10.115

10.130

$x^{1} \Sigma^{+}, v^{\prime}=4$

10.395

10.410 
Table II. Comparison of the tonization potentials of NO( $\left.x^{2} \pi: v^{n}=0\right)+10^{+}\left(x^{1} \Sigma^{+}, v^{\prime}=0\right)$

$\left(n_{3 / 2}, v^{\prime \prime}=0\right)+\left(x^{1} \Sigma^{+}, v^{\prime}=0\right) \quad\left(n_{1 / 2}, v^{\prime \prime}=0\right)+\left(x^{1} \Sigma^{+}, v^{\prime} m 0\right) \quad$ Mathod and Ref.

$9.256 \pm 0.003$

Photolonization

This work

$9.2637 \pm 0.0006$

Spectroscoptc

(2)

$9.267 \pm 0.005$

Spectroscopte

(26)

$9.266 \pm 0.008$

Spectroscopic (27)
$9.250 \pm 0.005$
$9.25 \pm 0.02$
$9.20 \pm 0.03$
$9.25 \pm 0.03$

Photolonization (9)

Photolonization (28)

Photoionization (29)

Photolonization

(8)

9.23

Photoelectron (30)

9.32

Photoelectron (3i)

9.34

Photoelectron (32)

9.25

Photoelectron (33)

$9.25 \pm 0.002$

Electron Impact (34) 
Table III. Vibrational Spacing of the Lowest Iontc State $\left(\Sigma^{+}\right)$of No

\begin{tabular}{lcccccc}
\hline$\Delta v^{\prime}$ & Calculated $^{\mathrm{a}}$ & Photoionization $^{\mathrm{b}}$ & Spectroscopic $^{c}$ & Electron Impact $^{\mathrm{d}}$ & Photoelectron $^{\mathrm{e}}$ This work $^{-}$ \\
\hline $0-1$ & 0.290 & 0.290 & 0.291 & 0.29 & 0.30 & 0.291 \\
$1-2$ & 0.287 & 0.288 & 0.286 & 0.26 & 0.29 & 0.287 \\
$2-3$ & 0.283 & 0.277 & 0.283 & & 0.28 & 0.281 \\
3.4 & 0.278 & 0.295 & 0.278 & 0.28 & 0.280
\end{tabular}

${ }^{\text {a Reference } 36}$

Beference 9

CReference 37

Reference 38

Eeference 31 
Table IV A. Assignment of Autotonization Structure on the First Vibrational step of $\mathrm{NO}^{+}\left(\mathrm{L}^{+}+2^{2} \mathrm{~m}_{3 / 2}\right)$

\begin{tabular}{|c|c|c|c|c|}
\hline \multirow{2}{*}{$\begin{array}{l}\text { Observed } \\
\qquad(\AA)\end{array}$} & \multirow{2}{*}{$\begin{array}{l}\text { Peaks Predicted } \\
\text { by Equation }(1)(\AA)\end{array}$} & \multirow[t]{2}{*}{$\delta$} & \multicolumn{2}{|c|}{ Assignment } \\
\hline & & & $n$ & $v^{\prime}$ \\
\hline 1336.67 & 1336.12 & 0.87 & 8 & 1 \\
\hline 1327.67 & 1327.31 & 0.87 & 9 & 1 \\
\hline 1321.17 & 1321.35 & 0.87 & 10 & 1 \\
\hline 1316.67 & 1316.95 & 0.87 & 11 & 1 \\
\hline 1313.17 & 1313.88 & 0.87 & 12 & 1 \\
\hline
\end{tabular}


Table IV B. Assignment of Autoionization Structure on the first Viurational step of $\mathrm{NO}^{+}\left(\Sigma^{+}+{ }^{2} \Pi_{1 / 2}\right)$

\begin{tabular}{ccccc}
\hline $\begin{array}{c}\text { Observed } \\
(\AA)\end{array}$ & $\begin{array}{l}\text { Peaks Predicted } \\
\text { by Equation (2) }(\AA)\end{array}$ & $\delta$ & \multicolumn{2}{c}{ Assignment } \\
& 1333.96 & 0.87 & 8 & 1 \\
1334.17 & 1325.18 & 0.87 & 9 & 1 \\
1325.17 & hv $=\left[9.561_{8}-\frac{13.607_{4}}{(n-0.87)^{2}}\right]$ ev & (2)
\end{tabular}


Table V A. Assignment of Autoionization Structure on the Second Vibrational step of $\mathrm{NO}^{+}\left(1 \varepsilon^{+}+{ }^{2} \mathrm{n}_{3 / 2}\right)$

\begin{tabular}{|c|c|c|c|c|c|}
\hline \multirow{2}{*}{$\begin{array}{c}\text { Observed } \\
\text { (A) }\end{array}$} & \multirow{2}{*}{\multicolumn{2}{|c|}{$\begin{array}{l}\text { Peaks Predicted } \\
\text { by Equation (3) (A) }\end{array}$}} & \multirow[t]{2}{*}{$\delta$} & \multicolumn{2}{|c|}{ Assignment } \\
\hline & & & & $n$ & $v^{\prime}$ \\
\hline 1296.17 & 1295.94 & & 0.87 & 8 & 2 \\
\hline 1297.67 & 1287.62 & & 0.87 & 9 & 2 \\
\hline 1281.67 & 1281.95 & & 0.87 & 10 & 2 \\
\hline 1277.67 & 1277.90 & & 0.87 & 11 & 2 \\
\hline 1275.17 & 1274.91 & & 0.87 & 12 & 2 \\
\hline
\end{tabular}


Table $\mathbf{V} 8$. Assignment of Autoionization Structure on the Second Vibrational step of $\mathrm{NO}^{+}\left(1_{\Sigma}^{+}+{ }^{2} \mathrm{I}_{1 / 2}\right)$

\begin{tabular}{ccccc}
\hline $\begin{array}{c}\text { Observed } \\
(\AA)\end{array}$ & $\begin{array}{l}\text { Peaks Predicted } \\
\text { by Equation (4) }(\AA)\end{array}$ & $\delta$ & \multicolumn{2}{c}{$\begin{array}{c}\text { Assignment } \\
n\end{array}$} \\
\hline 1293.67 & 1293.92 & 0.87 & 8 & 2 \\
1285.17 & 1285.63 & 0.87 & 9 & 2 \\
\hline & $h v=\left[9.849_{6}-\frac{13.607_{4}}{(n-0.87)^{2}}\right] \mathrm{ev}$ & (4)
\end{tabular}


Table VI A. Assignment of Autoionization Structure on the Third Vibrational Step of $\mathrm{NO}^{-+} \quad\left(\Sigma_{\Sigma}^{+}+{ }^{2} \mathrm{M}_{3 / 2}\right)$

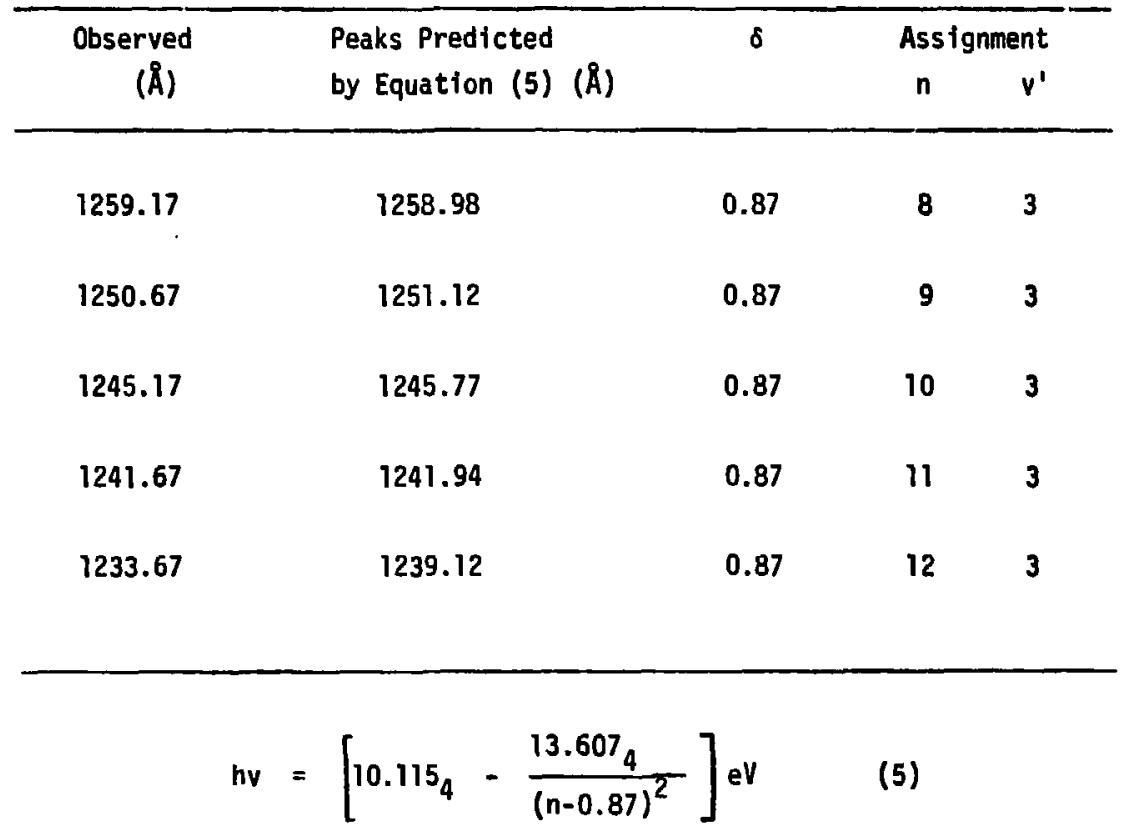


Table VI 8. Assignment of Autoionization Structure on the Third Vibrational step of $\mathrm{NO}^{+} \quad\left(\Sigma^{+}+{ }^{2} \mathrm{n}_{1 / 2}\right)$

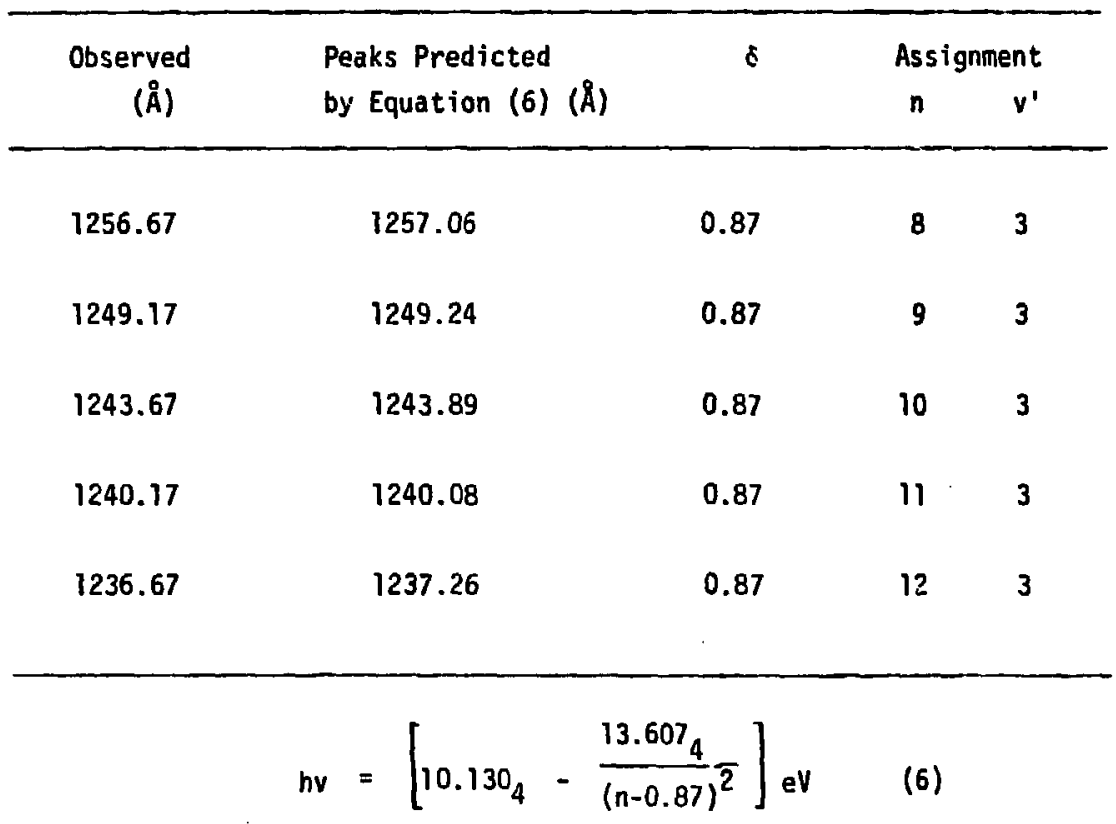


Table VII A. Assignment of Autoionization Structure on the Fourth Vibrational step on $\mathrm{NO}^{+} \quad\left({ }^{1} \Sigma^{+}+\pi_{3 / 2}\right)$

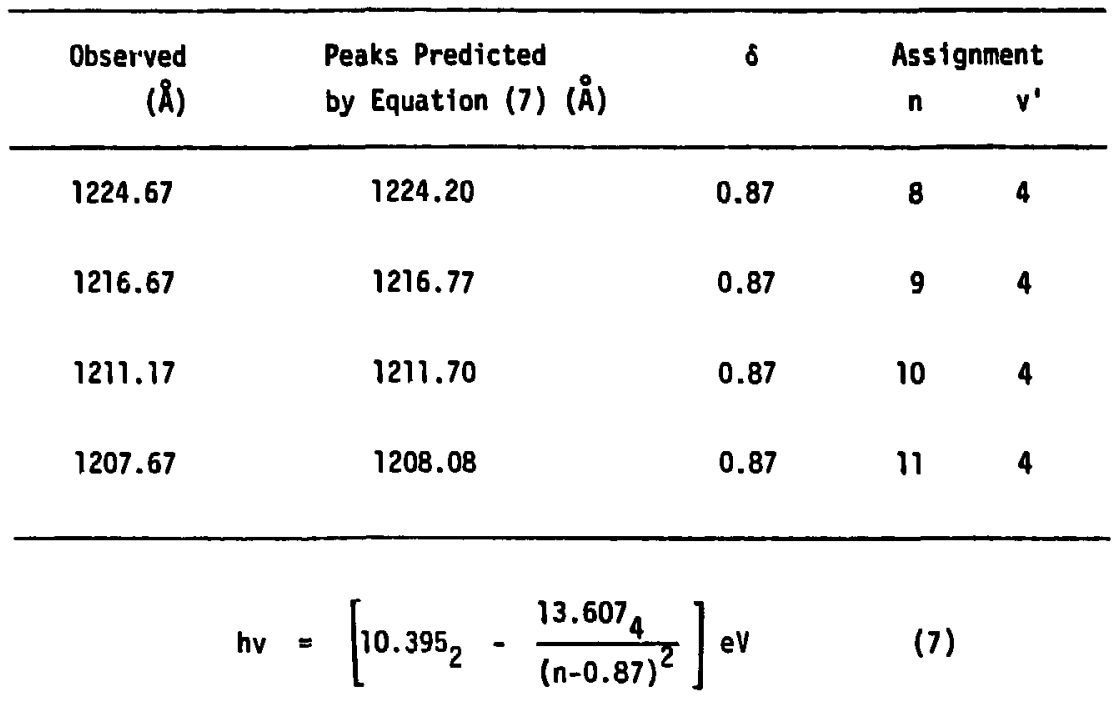


Table VII B. Assignment of Autoionization Structure on the Fourth Vibrational Step of $\mathrm{NO}^{+} \quad\left(\Sigma^{+}+{ }^{2} \pi_{1 / 2}\right)$

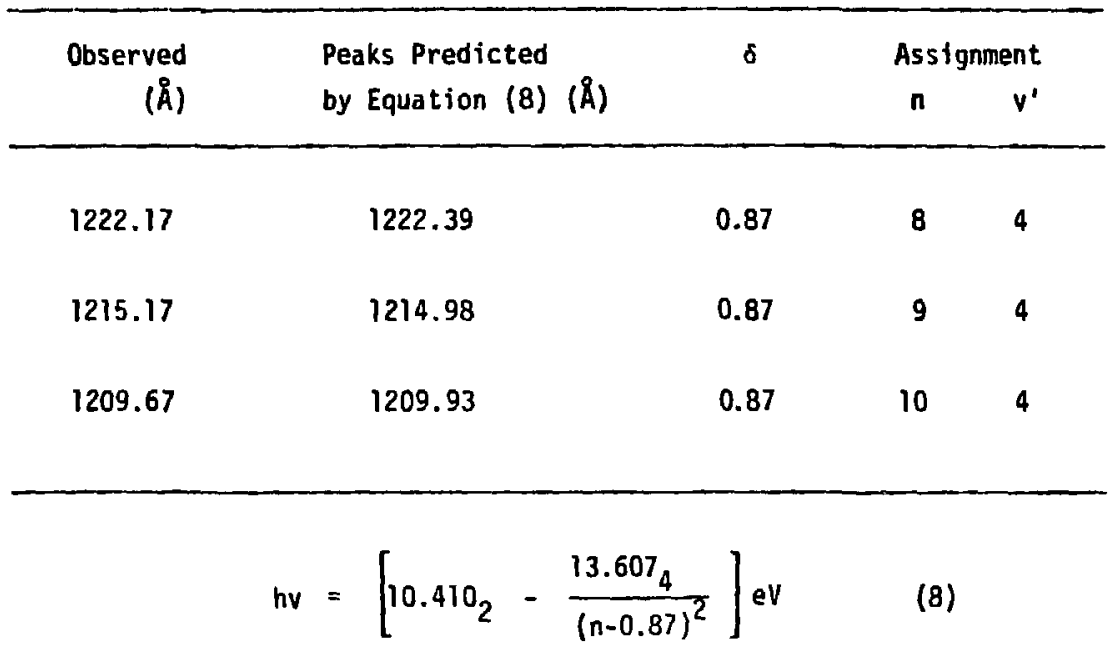


Table VIII. Relative Transition Probabilities (nomalized for $y^{\prime}=1$ )

\begin{tabular}{llcccc}
\hline$v^{\prime}$ & \multicolumn{2}{c}{ Calculated } & $\begin{array}{c}\text { Photoionization } \\
\text { Ref. 7 }\end{array}$ & $\begin{array}{c}\text { Photoelectron } \\
\text { Ref. 31 }\end{array}$ & This work \\
\hline 0 & 0.478 & 0.4781 & 0.79 & 0.59 & 0.70 \\
1 & 1.000 & 1.000 & 1.00 & 1.00 & 1.00 \\
2 & 0.917 & 0.9170 & 1.00 & 0.81 & 1.03 \\
3 & 0.484 & 0.4843 & 0.67 & 0.40 & 0.70 \\
4 & 0.163 & 0.1629 & & 0.16 & 0.28
\end{tabular}




\section{FIGURES}

Figure 1. Schematic diagram of the apparatus (Plane View)

(1) McPherson 225 Vacuum UV Monochromator,

(2) Differential Pumping System, (3) Entrance-

Slit, (4) Light Source, (5) Exit Slit, (6) Sodium

Salicylate Coated Quartz Window, (7) Photon

Detector, (8) Nozzle, (9) Tuning Fork Chopper.

Figure 2. Schematic diagram of the apparatus (Vertical Cross Section View) (1) Nozzle, (2) Skimmer, (3) Tuning Fork Chopper, (4) Quadrupole Mass Spectrometer,

(5) Focusing Ion Lenses, (6) Electron Gun.

Figure 3. Photoionization efficiency curve of $\mathrm{NO}^{+}\left(\mathrm{x}^{1} \Sigma^{+}\right)$in the wavelength range from $1350 \AA$ to $1185 \AA$ (9.18$10.46 \mathrm{eV})$.

Figure 4. The onset of the $\left(x^{l} \Sigma^{+}, v^{\prime}=0\right)$ state at different nozzle pressures. (a) 100 torr, (b) 300 torr, (3) 450 torr. 


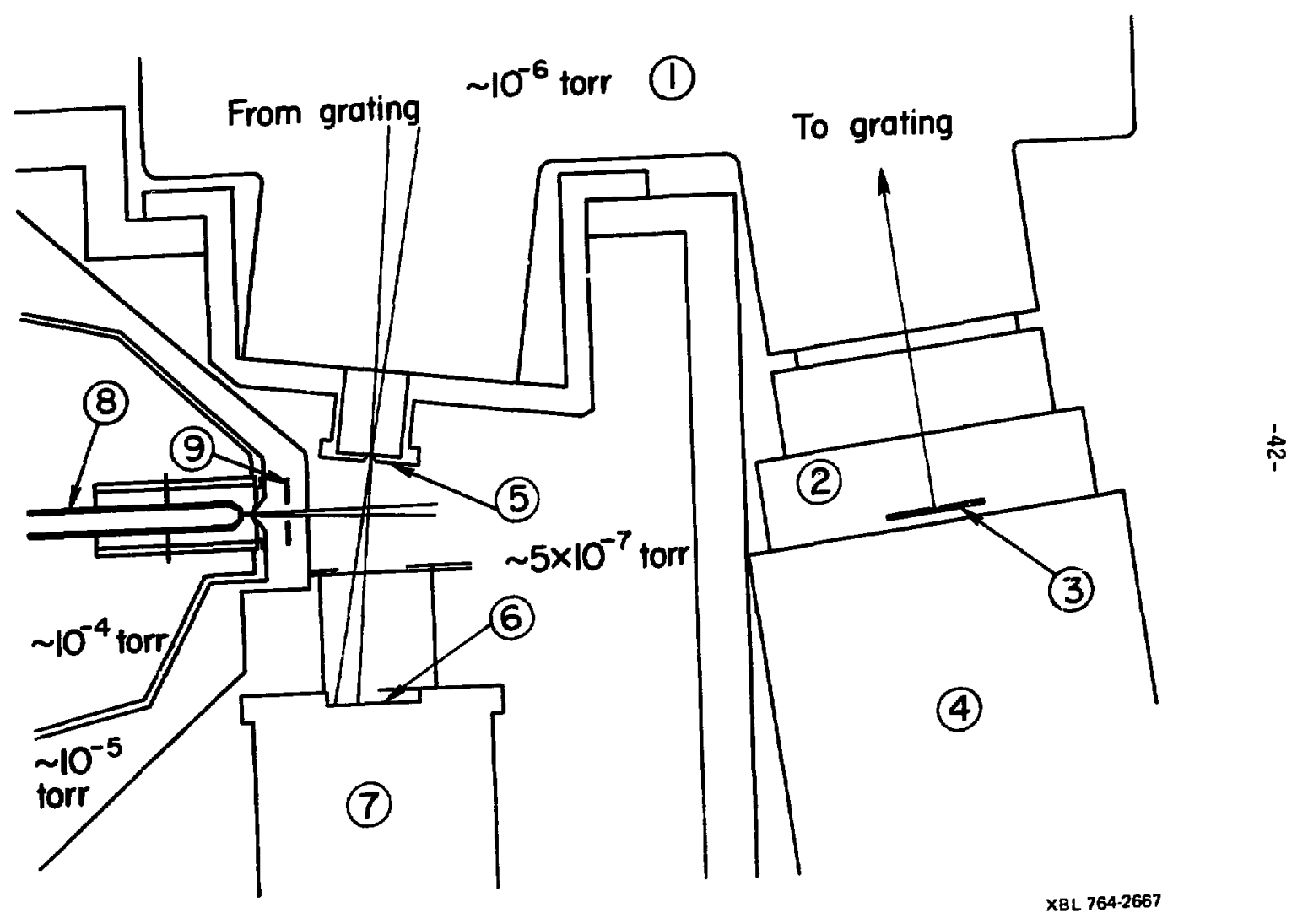

Fig. 1 


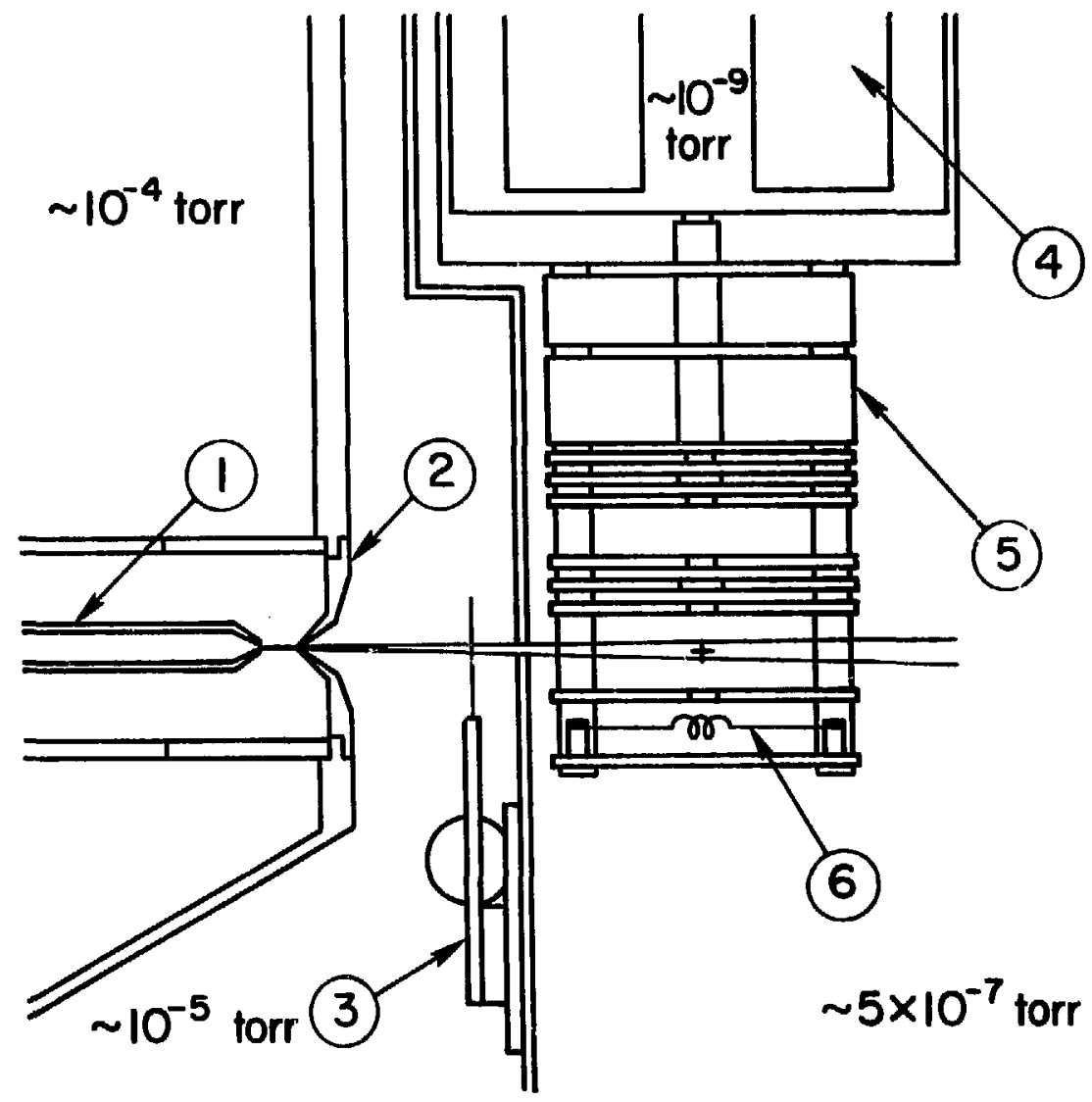

XBL 761-2666

Fig. 2 


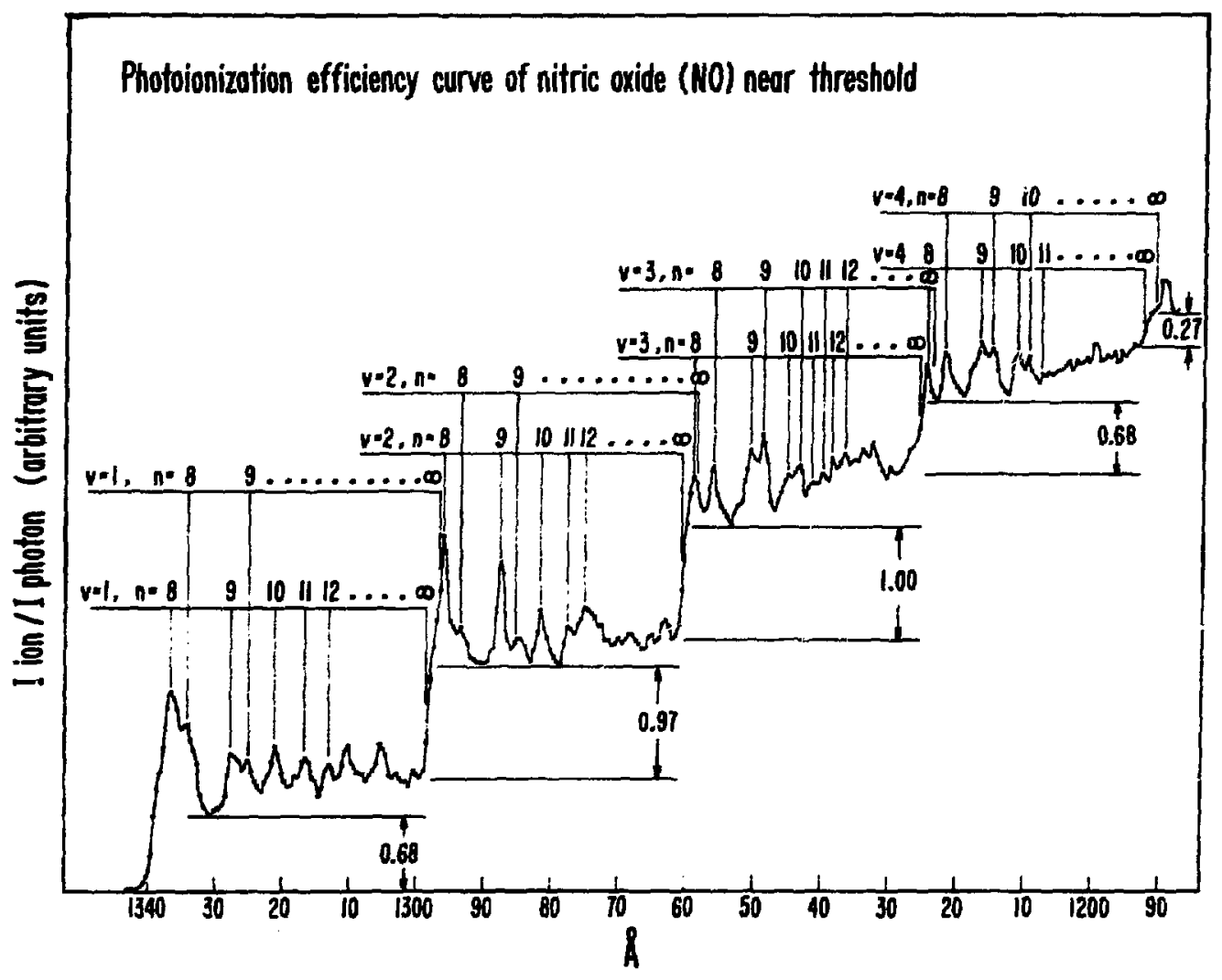

Fig. 3 


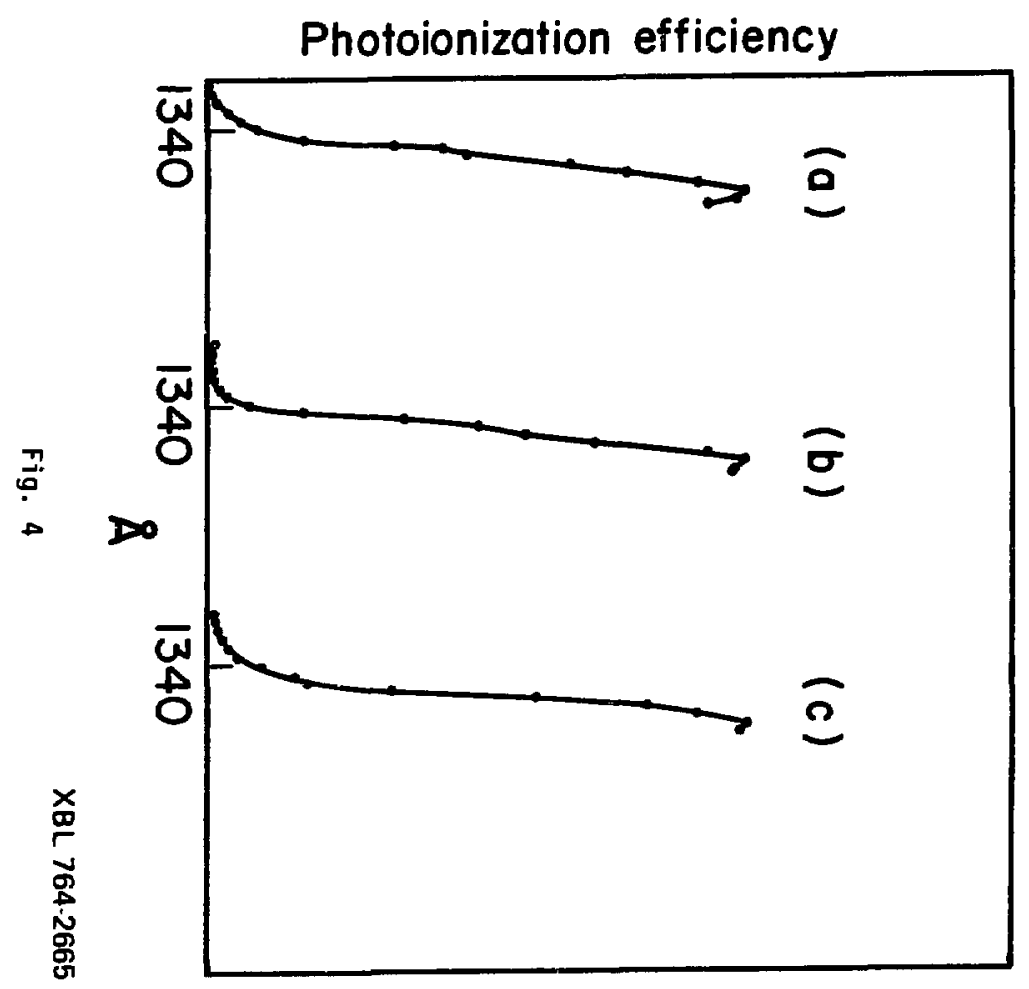


IV. THE BINDING ENERGY BETWEEN NO AND NO ${ }^{+}$

\section{ABSTRACT}

The photolonization efficiency curve of the nitric oxide dimer (NO) 2 was obtained in the wavelength range from $1420 \AA$ to $1230 \AA$ $(8.731 \mathrm{eV}-10.080 \mathrm{eV})$. The ionization potential of (NO) $)_{2}$ was found to be $8.752 \mathrm{eV} \pm 0.008 \mathrm{eV}$. From the structure of the photoion yield curve of $\left.(\mathrm{NO})_{2}, \mathrm{NO}^{2} \mathrm{II}, \mathrm{v}^{\prime \prime}=0\right)-\mathrm{NO}^{+}\left(\mathrm{X}^{1} \Sigma^{+}, v^{\prime}\right)$ is found to be bound when $\mathrm{NO}^{+}\left(X^{1} \Sigma^{+}\right)$is in $v^{\prime}=0$ or 1 vibrational state. From this and the dissociation energy of $\left(\mathrm{NO}_{2}(0.069 \mathrm{eV} \pm 0.003 \mathrm{eV})\right.$ obtained by Billingsley and Callear, the dissociation energy of $\left.\mathrm{NO}^{2} \Pi, v^{\prime \prime}=0\right)$ $\mathrm{NO}^{+}\left(X^{1} \Sigma^{+},{ }^{\prime}=0\right)$ is deduced to be $0.573 \mathrm{eV} \pm 0.008 \mathrm{eV}$. 


\section{INTRODUCTION}

The important role played by the nitric oxide dimer in many atomic and molecular processes has been recognized recently. For example, the chemiluminous reaction (NO) $)_{n}+\mathrm{O}+\mathrm{NO}_{2}^{*}+(\mathrm{NO})_{n-1}$ is found to be several orders of magnitude faster than the three body reaction of $\mathrm{NO}+\mathrm{O}+\mathrm{M}+\mathrm{NO}_{2}^{*}+\mathrm{M}^{1-3}$. Both the rapid vibrational relaxation of nitric oxide ${ }^{4}$ and anomalous thermodynamic properties of gaseous nitric oxide at low temperatures ${ }^{5-8}$ are attributed to the existence of $(\mathrm{NO})_{2}$. The nitric oxide dimer $(\mathrm{NO})_{2}$ in the gas phase has been directly observed by mass spectrometric sampling of an expanding No jet $^{9-10}$ using an electron bombardment type ionizer. From studies of the ultraviolet spectrum $11-12$ and infra-red transitions of the dimer in the gas phase, ${ }^{13}$ the heat of formation of the dimer has been estimated to be $2.45 \mathrm{kcal} / \mathrm{mol}$. The structure of the nitric oxide dimer $\left(\mathrm{NO}_{2}\right.$ has been determined in the gas phase by infrared measurements, ${ }^{13}$ and in the solid state by $x$-ray crystallography. ${ }^{14-15}$ It is found that $\left(\mathrm{NO}_{2}\right.$ has a nearly rectangular cis configuration. In this work we report the first photoionization study of $(\mathrm{NO})_{2}$. One of the main reasons for this study was to determine the binding energy between $\mathrm{NO}$ and $\mathrm{NO}^{+}$. If the ionization.mechan $1 \mathrm{sm}$ of $(\mathrm{NO})_{2}$ is such that only onie of the NO molecules in the dimer is being excited to the different vibrational states of $\mathrm{NO}^{+}\left(\mathrm{X}^{1} \Sigma^{+}\right)$, we would expect to see the same stepfunction behavior that is observed in the photoionization efficiency curve of NO alone. Furthermore, since the intermolecular lergy transfer in the $N O-N O^{+}\left(\Sigma^{+} v^{\prime}\right)$ complex is expected to be faster than the ion 
transit time to the detector $\left(-10^{-6} \mathrm{sec}\right)$, if the energy of a certain vibrational level of $\mathrm{NO}^{+}\left({ }^{1} \Sigma^{+}\right)$is greater than the binding energy of NO-NO ${ }^{+}$, the complex will no longer be stable. Thus the number of steps observed in the photoionization efficiency curve will be related to the binding energy of $\mathrm{NO}^{-} \mathrm{NO}^{+}\left({ }^{+}{ }^{+}\right)$. It is with this expectation that the photoionization study of $(\mathrm{NO})_{2}$ has been carried out.

\section{EXPERIMENTAL}

The experimental apparatus is essentially the samis as that described previously. ${ }^{16}$ Modifications were made such that the system can now be operated automatically. ${ }^{17}$ A stepping motor was installed in the McPherson 225 one meter monochromator. When the machine was operated in the automatic mode, the ion counters and the photon counter counted for a preset time at a certain wavelength and then the signals were printed out by a printer. Immediately after the printing, the stepping motor advanced upward or downward by a preset number of steps which corresponded to a certain increment of wavelength, and the scalers were reset and restarted simultaneously. The hydrogen many-line pseudocontinuum was used as the light source. The grating used was coated with $\mathrm{MgF}_{2}$ and has $1200 \mathrm{lines} / \mathrm{m} . \mathrm{m}$. With the $300 \mathrm{\mu}$ entrance and exit slits used in this experiment, the resolution was about $2.49 \AA$ (i.e. $-16 \mathrm{meV}$ at $1400 \AA$ ). The nitric oxide used in this experiment was C.P. grade (99\% minimum in purity) obtained from Matheson without further purification. A nozzle $0.127 \mathrm{~mm}$ in diameter was used. The photo- 
ionization efficiency curve of ( $\mathrm{NO}_{2}$ was obtained with a nozzle stagnation pressure of 600 torr. The monochromator was scanned automatically at $1 \AA$ intervals, and at each wavelength, the scalers were set to count for $300 \mathrm{sec}$. The counting rates varied from about $0.5 \mathrm{count} / \mathrm{sec}$ to 5 counts/sec. Wavelength calibration was accomplished by using the known emission lines of the hydrogen atom and molecule. The photon signal has been corrected for the background of stray photons from the monochromator.

\section{RESULTS AND DISCUSSION}

The percentage of concentration of nitric oxide dimer versus nozzle stagnation pressure of nitric oxide at room temperature was obtained at $1215.7 \AA$ (Lyman $\alpha$ ). The results shown in Fig. 1 are calculated with the assumption that the photoionization cross section of $\left(\mathrm{NO}_{2}\right.$ is twice that of NO. It has been shown that when a gas is adiabatically expanded from a high pressure gas reservior into a vacuum, association of the molecules occurs as a first step in the condensation process. ${ }^{18-19}$ Due to adiabatic cooling, the gas jet after expansion consists of a higher percentage of dimer as compared to the percentage before expansion. For a given gas, the degree of cooling through the expansion depends strongly on the parameter $P_{0} \cdot D_{0}$, where $P_{0}$ is the nozzle stagnation pressure and $D_{0}$ is the diameter of the nozzle. It is seen that the concentration of the $\left(\mathrm{NO}_{2}\right.$ dimer relative to the monomer, NO, rises with increasing pressure. No attempt has been made to analyze the dimer formation in 
the expanding gas beam of NO. However, it was found that with the present beam production arrangement, the concentration of $\left(\mathrm{NO}_{2}\right.$ is approximately proportional to $\mathrm{P}_{0}^{2}$.

The photoion yield spectrum of $\left(\mathrm{NO}_{2}\right.$ obtained is shown in Fig. 2 . The photoionization efficiency curve of NO is also plotted for comparison. The ionization potential of $\left(\mathrm{NO}_{2}\right)_{2}$ obtained in this work is equal to $8.752 \mathrm{eV} \pm 0.008 \mathrm{eV}$, which is $0.504 \mathrm{eV} \pm 0.008 \mathrm{eV}$ lower than the I.P. of the NO molecule $\mathrm{e}^{16}(9.256 \mathrm{eV} \pm 0.003 \mathrm{eV})$. The standard deviation of the photoionization efficiency at several points is shown in Fig. 2. Due to the small counting rate of $(\mathrm{NO})_{2}^{+}$, the average standard deviation of this spectrum is as high as $15-20 \%$. This prevents us from analyzing the fine structure of the spectrum.

The photoionization efficiency curve of NO has been found to follow predominately a step-function behavior which corresponds to direct ionization of NO $\left(v^{\prime \prime}=0\right)$ to $\mathrm{NO}^{+}\left(\mathrm{X}_{\Sigma}^{1} \Sigma^{+}, v^{\prime}=0,1,2,3,4\right.$ etc $)$. Although the stability of $(\mathrm{NO})_{2}$ is attributed to a weak "chemical bond" which arises from electron pairing between two $\left({ }^{2} \Pi\right)$ No molecules, the coupling between the two interacting NO molecules will still be weak. The unpaired electron will mainly be localized on each NO molecule. Thus when a photon comes in and knocks out an electron, the fonization process is expected to be similar to that of NO alone. The only difference is that the ionization potential of the $(\mathrm{NO})_{2}$ will be shifted to lower energy due to the interaction of the ion with its partner No molecule. However, in the photoion yield curve of $\left(\mathrm{NO}_{2}\right.$, only two steps are clearly resolved. They are separated by $0.290 \mathrm{eV}$, which is the vibrational quantum of the NO molecule. 
In order to explain the observed features in the photoionization efficiency curve of (NO), schematic representations of the potential energy curves for $\left(\mathrm{NO}_{2}\right.$ and $(\mathrm{NO})_{2}^{+}$are shown in Fig. 3. The quantities $\varepsilon$ and $\varepsilon^{+}$are the dissociation energies of NO-NO and $\mathrm{NO}^{-} \mathrm{NO}^{+}$, respectively. The ionization process of $\left\langle\mathrm{NO}_{2}\right.$ involves ejecting an antibonding electron which is associated with one of the No molecules. This can result in the excitation of the stretching vibrational mode of $\mathrm{NO}^{+}$. The onset of the first step in the photoion yield curve (i.e. the I.P. of $(\mathrm{NO})_{2}$ ) can be attributed to the ionization threshold of $\left(\mathrm{NO}_{2}\right.$ to form $\mathrm{NO}-\mathrm{NO}^{+}(v=0)$. The onset of the second step is then equal to the I.P. of $(\mathrm{NO})_{2}$ to form $\mathrm{NO}-\mathrm{NO}^{+}(v=1)$. With this model, the nonexistence of the third step in the photoion yield curve implies that $\mathrm{NO}^{-\mathrm{NO}^{+}}(\mathrm{v}=2)$ has an energy larger than the ion-neutral bond energy. Intramolecular energy transfer is expected to be fast and the dimer dissociates in less than $-10^{-6} \sec$ (i.e. the estimated flight time from the ionizing region to the entrance of the quadrupole mass spectrometer). Thus we have

$$
(\mathrm{NO}-\mathrm{NO})+\mathrm{hv} \longrightarrow \mathrm{NO}-\mathrm{NO}^{+}(\mathrm{V}=2) \stackrel{\leq=10^{-6} \mathrm{sec}}{\longrightarrow} \mathrm{NO}+\mathrm{NO}^{+}+\text {K.E. }
$$

The charge exchange cross section between the neutral no molecule and the $\mathrm{NO}^{+}$molecular ion is expected to be large. This does not change any of the argument presented above. The fact that $\mathrm{NO}^{-\mathrm{NO}^{+}}$( $\left.\mathrm{V}=1\right)$ is bound but $\mathrm{NO}^{-\mathrm{NO}^{+}}(\mathrm{V}=2)$ is not, permits a measurement of the well depth of $\left(\mathrm{NO}-\mathrm{NO}^{+}\right)$. The minimum well depth for the NO and $\mathrm{NO}^{+}$interaction is $0.290 \mathrm{eV} \pm 0.008 \mathrm{eV}\left(\varepsilon_{\min }^{+}\right)$and the maximum is estimated to be $0.580 \mathrm{eV} \pm 0.008 \mathrm{eV}\left(\varepsilon_{\max }^{+}\right)$, which correspond to the vibrational 
energies of $\mathrm{NO}^{+}(\mathrm{v}=1)$ and $\mathrm{NO}^{+}(\mathrm{v}=2)$, respectively. From Fig. 3 , the following relation can be derived:

$$
\varepsilon^{+}+\operatorname{I.P.}\left(\left(\mathrm{NO}_{2}\right)=\text { I.P.(NO) }+\varepsilon\right.
$$

Using $\varepsilon_{\max }^{+}$and substituting into equation (1), an upper bound for the dissociation energy of $\left(\mathrm{NO}_{2}\right.$ is found to be $0.076 \mathrm{eV} \pm 0.008 \mathrm{eV}$. On the other hand, with the value of $0.069 \mathrm{eV} \pm 0.004 \mathrm{eV}$ for the dissociation energy of (NO) 2 as determined by Billingsley and Callear. we would obtain a value of $0.573 \mathrm{eV} \pm 0.008 \mathrm{eV}$ for the dissociation energy of $\left(\mathrm{NO}-\mathrm{NO}^{+}\right)$. This certainly lies within our estimates.

\section{ACKNOWLEDGEMENTS}

This research was supported by the Office of Naval Research and the U. S. Energy Research and Development Administration. 


\section{REFERENCES}

1. A. Fontijin and D. E. Rosner, J. Chem. Phys., 46, 3275 (1967).

2. D. Golomb and R. E. Good, J. Chem. Phys., 49, 4176 (1968).

3. M. Bodenstein, Helv. Chim. Acta, 18, 745 (1935).

4. J. Billingsley and A. B. Callear, Nature, 221, 1136 (1969).

5. O. K. Rice, J. Chem. Phys., 4, 367 (1936).

6. R. L. Scott, Mol. Phys., 11, 399 (1966).

7. E. A. Guggenheim, Mol. Phys., 10, 401 (1966).

8. E. A. Guggenheim, Mol. Phys., 11, 403 (1966).

9. T. A. Milne and F. T. Greene, J. Chem. Phys., 47, 3668 (1967).

10. R. E. Leckenby and E. J. Robbins, Nature, 207, 1253 (1965); Proc. Roy. Soc., (London) 291A, 389 (1966).

11. J. Billingsley and A. B. Callear, Trans. Faraday Soc., 67, 589 (1971).

12. L. D'Or, A. deLattre and P. Tarte, J. Chem. Phys., 19, 1064 (1951).

13. C. E. Dinerman and G. E. Ewing, J. Chem. Phys., 53, 626 (1970).

14. W. J. Dulmage, E. A. Meyers and W. N. Lipscomb, Acta Cryst., 6, 760 (1953).

15. W. N. Lipscomb, F. E. Wang, W. R. May and E. Lippert, Jr., Acta Cryst., 14, 1100 (1961).

16a. C. Y. Ng, B. H. Mahan and Y. T. Lee, J. Chem. Phys,, to be publtshed.

b. C. Y. Ng, D. J. Trevor, B. H. Mahan and Y. T. Lee, J. Chem. Phys., to be published.

17. C. Y. Ng, Ph.D. Thesis, University of California, Berkeley (1976).

18. F. T. Greene and T. A Milne, J. Chem. Phys., 39, 3150 (1963).

19. R. E. Leckenby, E. J. Robbins and P. A. Trevalion, Proc. Roy. Soc. A, 280, 409 (1964). 


\section{FIGURES}

Fig. 1. Variation of concentration of nitric oxide dimer with nozzle stagnation pressure at room temperature as probed at Lyman $\alpha$

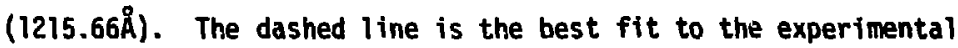
points, and is given by $\%$ of $(\mathrm{NO})_{2}=\left(7.97 \times 10^{-4}\right)\left[\mathrm{P}_{0} \cdot \mathrm{D}_{0}\right]^{2}$ where $P_{0}$ is the nozzle stagnation pressure in torr, and $D_{0}$ is the nozzle diameter $(0.0127 \mathrm{~cm})$.

Fig. 2. Photoionization efficiency curve of $(\mathrm{NO})_{2}$ in the wavelength range from $1230 \AA$ to $1425 \AA(10.080 \mathrm{eV}-8.700 \mathrm{eV})$.

Fig. 3. Schematic representation of $\mathrm{NO}+\mathrm{NO}$ and $\mathrm{NO}+\mathrm{NO}^{+}$potential energy curves. $\varepsilon^{+}$and $\varepsilon$ denoce the dissociation energies of $(\mathrm{NO})_{2}^{+}$and $(\mathrm{NO})_{2}$, respectively. 


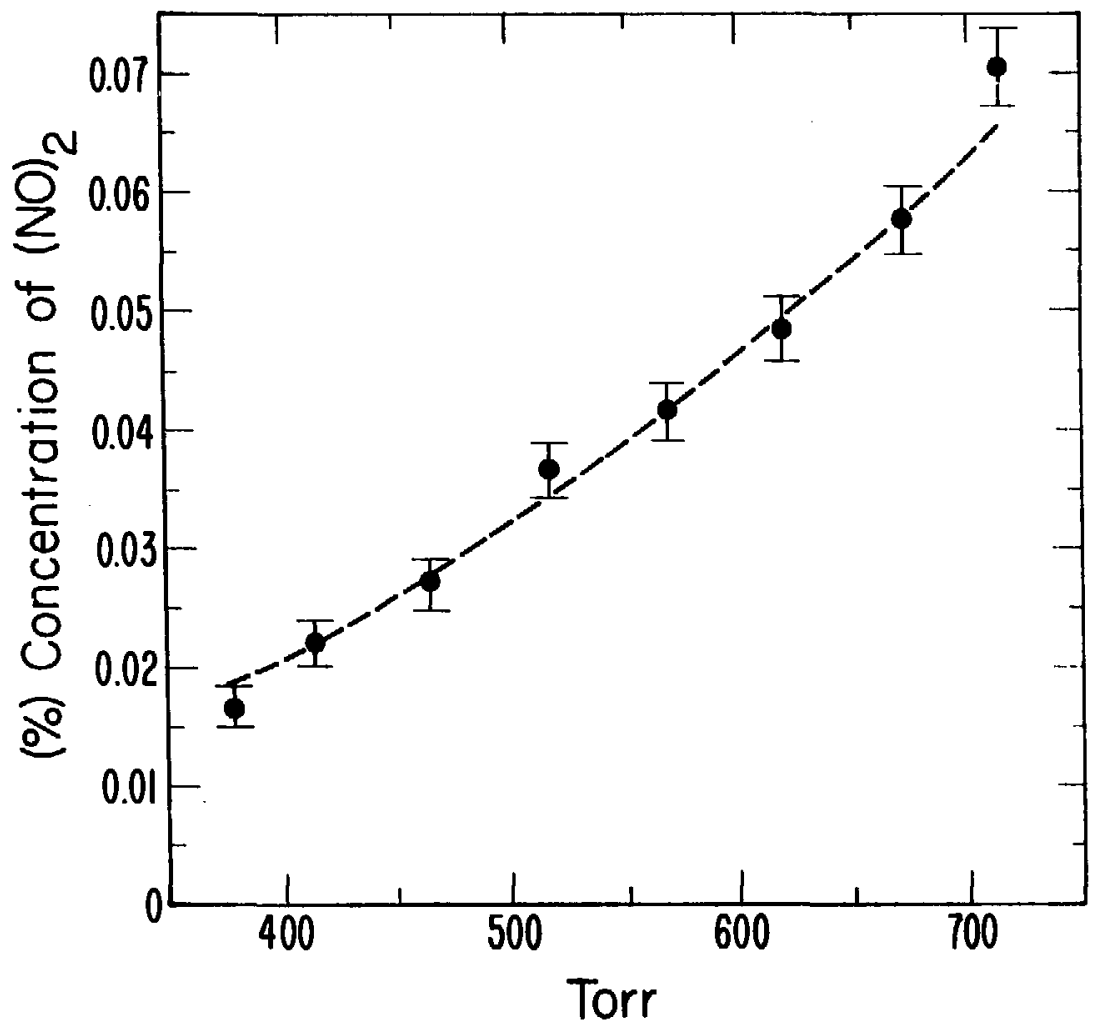

XBL 767-3201

Fig. 1 


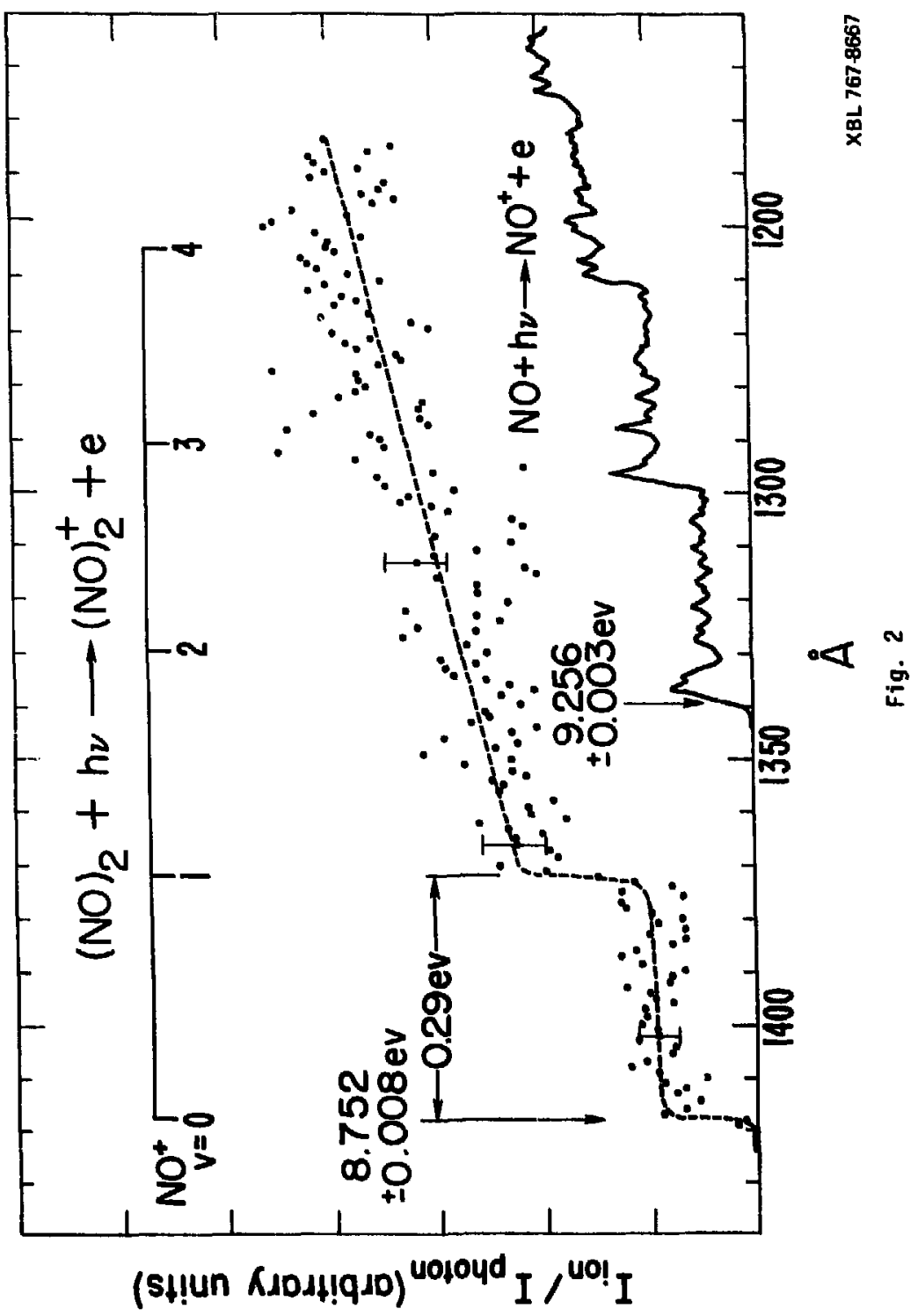




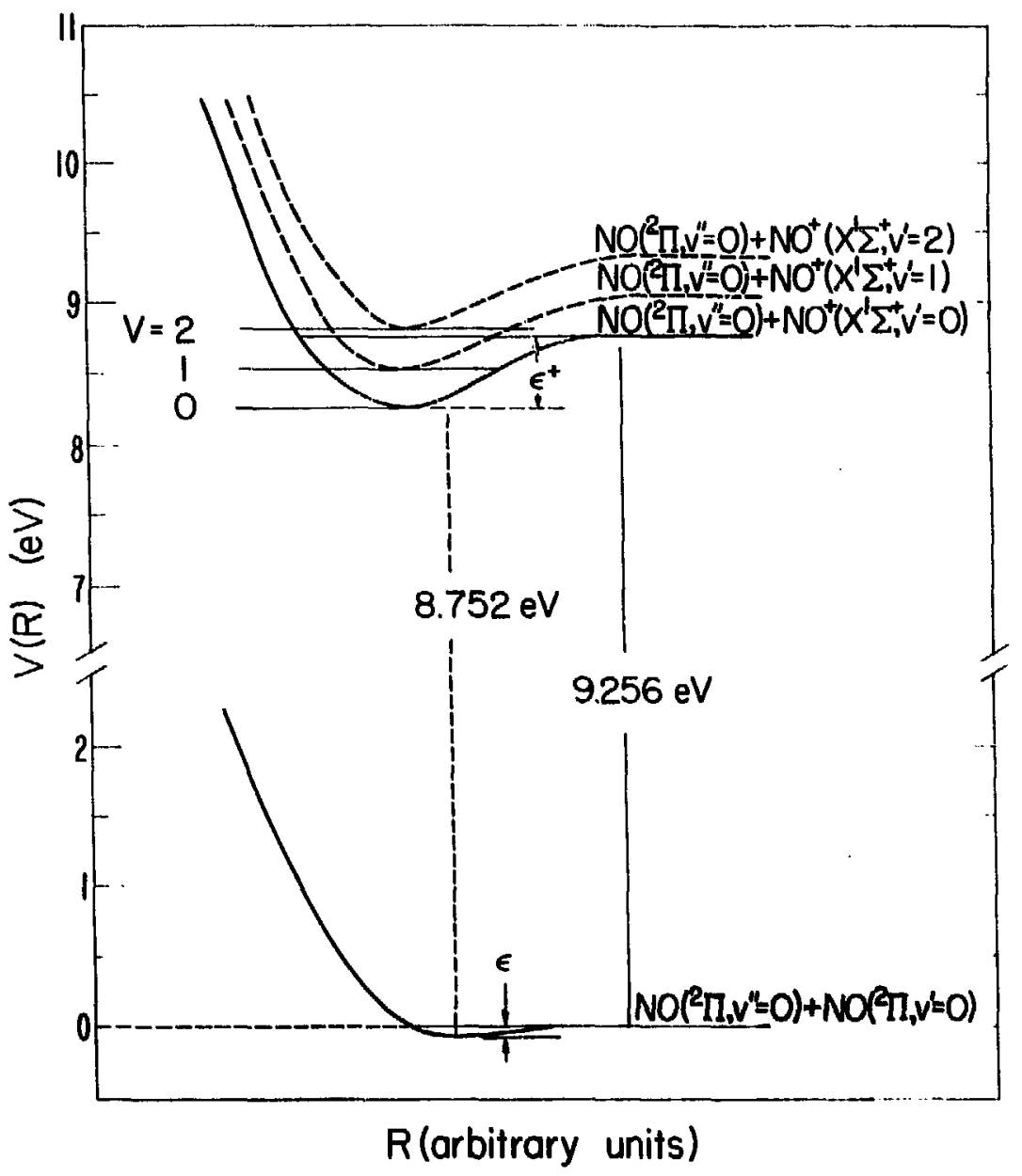

Fig. 3 
v. PHOTOIONIZATION STUDIES OF THE $\mathrm{Xe}_{2}, \mathrm{Kr}_{2}$ AND $\mathrm{Ar} r_{2}$ VAN DER WALLS MOLECULES

A. Photoionization Study of the $\mathrm{Xe}_{2}$ Van Der Waals Molecule*

The dissociation energy $D_{0}$ of the $X_{2}^{+}$ion has been estimated from mass spectrometric electron impact appearance potentials ${ }^{1,4}$ and photoionization methods. ${ }^{2,3}$ In the work of Huffman and Katayama, ${ }^{2}$ the lower bound for $D_{0}$ was estimated to be $0.967 \mathrm{eV}$ from the difference between the threshold energy of the photon induced associative ionization process $\mathrm{Xe}^{*}+\mathrm{Xe} \rightarrow \mathrm{Xe}_{2}^{+}$and the ionization potential of $\mathrm{Xe}$. In the work of Samson and Cairns, ${ }^{3}$ the ionization potential of $X_{2}$ was obtained by photoionization in a high pressure gas cell, where the mechanism of producing $\mathrm{Xe}_{2}^{+}$was predominately the same associative ionization process. From such experiments $D_{0}$ was found to be $0.99 \pm 0.02 \mathrm{eV}$. In this work, we report the first direct photoionization studies of the van der Waals molecule $\mathrm{Xe}_{2}$.

The experimental apparatus and procedures were essentially the same as previous described. 5 Brtefly, the apparatus consisted of a hydrogen lamp, a vacuum ultraviolet monochromator, a quadrupole mass spectrometer and a modulated molecular beam production system. The light intensity was monitored by a sodiun salicylate coated photomultiplier. The spectrometer grating is ruled with $1200 \mathrm{lines/mm}$ and has a reciprocal dispersion of $8.3 \AA / m m$. The $X_{2}$ van der Waals molecules were prepared by supersonic expansion of Xe through a -0.005 " diameter nozzle with a stagnation pressure of -350 torr at room temperature. The beam of $\mathrm{Xe}_{2}$

*Submitted to Journal of Chemical Physics for publication. 
molecules together with Xe atoms then intersected the dispersed vacuum ultraviolet photon beam at a distance of approximately 3 inches from the nozzle, after two stages of differential pumping. The ions produced were focused and mass analyzed. The number density at the collision center as probed by an electron gun was approximately $1.5 \times$ $10^{12}$ atom/cc for $X e$. The counting rate of $X_{2}^{+}$at $1020 \AA$ was about $10 \%$ that of $\mathrm{Xe}^{+}$. Assuming the photoionization cross section of $\mathrm{Xe}_{2}$ is twice that of $X e$, the $X_{2}$ molecules formed comprise roughly $5 \%$ of the beam $\left(-7.5 \times 10^{10}\right.$ molecule/cc $)$.

The photoionization efficiency curve obtained for $\mathrm{Xe}_{2}$ is shown in Fig. $1(a)$ and $1(b)$. In Fig. 1(a), the photoion yield curve was obtained with $300 \mu$ entrance and exit slits corresponding to a resolution of $2.5 \AA(-25 \mathrm{meV})$. Data were taken at intervals of $1 \AA$, counts being collected for $100 \mathrm{sec}$ at each point from $965 \AA$ to $1100 \AA$, while each point was counted for $200 \mathrm{sec}$ in the range from $1110 \AA$ to $1118 \AA$ where the photolonization efficiency is low. Counting rates varied from 100 counts $/$ sec to -0.5 counts $/ \mathrm{sec}$. Between $965 \AA$ and $1080 \AA$, the standard deviations were better than $4 \%$. No ions were observed at wavelengths greater than $1114.3 \AA$, and this can be taken to be the adiabatic Ionization potential of $X e_{2}$, with an uncertainty of $\pm 1 \AA$. This value together with the ionization potential of Xe and dissociation energy of $\mathrm{Xe}_{2}(-24 \mathrm{meV})^{6}$ gives $1.03 \pm 0.01 \mathrm{eV}$ as the dissociation energy $\mathrm{D}_{0}$ for $\mathrm{Xe}_{2}^{+}$. We note that our result is somewhat larger than the value given by Samson and Cairns. 3

The photoionization efficiency of $\mathrm{Xe}_{2}^{+}$increases very slowly from the threshold up to $1080 \AA$, and then increases dramatically with the 
autoionization structure dominating the spectrum. A small franckCondon factor for direct ionization is to be expected, since the ground state of $\mathrm{Xe}_{2}$ is essentially a repulsive state with a shallow van der Waals well ( -24 meV) and an equilibrium internuclear distance $\left(R_{e}\right)$ of approximately $4.4 \AA,{ }^{6}$ whereas the $X_{2}^{+}$ground state is bound with $R_{e}$ estimated to be about $2.85 \AA .{ }^{7}$ The anount of $\mathrm{Xe}_{2}^{+}$produced by associative ionization of noymal and excited $X e$ atoms in the beam is negligible under our experimental conditions. The photoion yield curve does not show the same structure as the work of Huffman and Katayama. ${ }^{2}$ This indicates that the $\mathrm{Xe}_{2}^{+}$we observed is not formed by secondary processes, but rather by $\mathrm{Xe}_{2}$ molecules which are synthesized by supersonic expansion.

In order to examine the coupling between the molecular excited Rydberg states and the molecular ionic states, of $\mathrm{Xe}_{2}$, the photoionization efficiency curve was measured again with $100 \mu$ exit and entrance slits (i.e. -10 meV resolution). With these narrower slits, the counting rate at a particular wavelength setting decreased by nearly one order of magnitude. Data were taken at $0.5 \AA$ intervals with a 200 sec counting time for each point. The results are shown in Fig. 1(b), where much detailed structure is evident. This spectrum was scanned twice and all the structure was found to be reproductble. In order to verify the wavelength calibration, the ionization threshold of $\mathrm{Xe}^{+}$was aiso measured and the data is shown in Fig. 1(a). The ionization potential of atomic xenon in this work is found to be $1022.3 \AA$ which is in good agreement with spectroscopic values. ${ }^{8}$ The standard deviations at several points are shown on the graph. 
An attempt was made to assign the excited atomic levels to which the molecular Rydberg states are correlated upon dissociation. In the Franck-Condon transition region of $X_{2}$, i.e. the neighborhood of the equilibrium distance for the ground state, the molecular states should correspond to the Hund-Mulliken case (c)..$^{9}$ The ground state of $\mathrm{Xe}_{2}$ has closed electronic shells, and thus is a $\mathrm{O}_{\mathrm{g}}^{+}$state. By the selection rules of case $(c)$, transticions for $0_{g}^{+}$to $0_{u}^{+}$or $1_{u}$ excited molecular states are the only ones which are electric dipole allowed. All the excited atomic states (except these with $\mathrm{J}=0$ ) can couple with the ground state $X e$ atom $\left(5_{p}{ }^{6} 1_{S_{0}}\right)$ to give an $0_{u}^{+}$and/or an $1_{u}$ molecular state. This makes the assignmerits of excited atomic states to the corresponding excited molecular stites nearly impossible within the present resolution of our experiment.. Only the electric dipole al lowed atomic Rydberg series ${ }^{8}$ are shown in Fig. 1(b). For example, between the $105\left[\frac{3}{2}\right]^{\circ}$, and $8 d\left[\frac{1}{2}\right]^{\circ}$, where the strongest observed autoionization peak is located in this spectrum, there exist a total of 14 wther excited atomic levels which can couple with the ground atomic state to give excited molecular states $l_{u}$ and/or $0_{u^{+}}^{+}$The number of possible atomic states increases as one approaches the ionization threshold of the xenon atom.

\section{ACKNOWLEDGEMENT}

This work was done with support from the U. S. Energy Research and Development Administration. 


\section{REFERENCES}

1. M. S. B. Munson, J. L. Franklin, and F. H. Field, J. Phys. Chem., 67. 1542 (1963).

2. R. E. Huffman and D. H. Katayama, J. Chem. Phys., 45, 138 (1966).

3. J. A. R. Samson and R. B. Cairns, J. Opt. Soc. Am., 56, 1140 (1966).

4. J. A. Hornbeck and J. P. Molnar, Phys. Rev., 84, 621 (1951).

5. to be published in J. Chem. Phys.

6a. J. A. Baker, R. O. Watts, U. K. Lee, T. P. Schafer and Y. T. Lee, J. Chem. Phys., 61, 308 (1974).

b. G. C. Maitland and E. B. Smith, Chem. Phys. Lett., 22, 443 (1973).

7. R. S. Mulliken, J. Chem. Phys., 52, 5170 (1970).

8. C. E. Moore, Atomic Energy Levels, NBS Circ., 467, Vol. III, (1949).

9. R. S. Mulliken, Rev. Mod. Phys., 3, 89 (1931); 4, 1 (1932). 
$-63-$

FIGURE CAPTIONS

Fig. 1. Phototonization efficiency curve of $\mathrm{Xe}_{2}$. (a) Phototon yield curve obtained with $300 \mu$ entrance and exit slit.

(b) Photoion yield curve obtained with $100 \mu$ entrance and exit slit. 
(a)

$x e_{2}+h \nu \rightarrow x e_{2}^{+}+e^{-}$

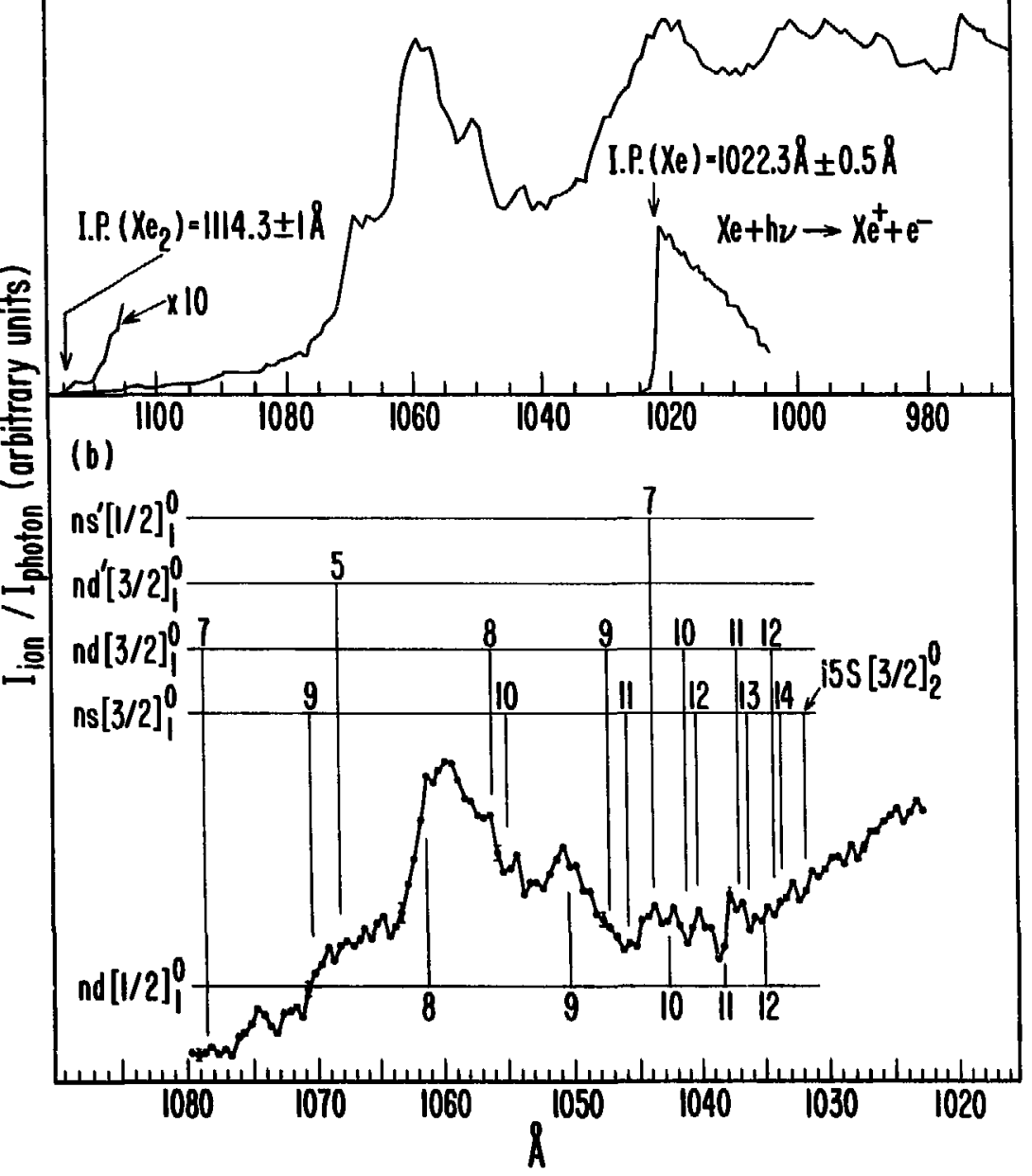

Fig. 1 
B. Photoionization Studies of the $\mathrm{Kr}_{2}$ and $\mathrm{Ar}_{2}$ Van Der Waals Molecules*

\section{ABSTRACT}

The photoionization efficiency curves of the $\mathrm{Kr}_{2}$ and $\mathrm{Ar}_{2}$ van der Waals dimers were obtained with the molecular beam technique in the wavelength range from 850 to $965 \AA(12.848-14.586 \mathrm{eV})$ and from 750 to $855 \AA$ ( $14.501-16.531 \mathrm{eV})$ respectively. The ionization potential of $\mathrm{Kr}_{2}$ was found to be $12.86 \pm 0.015 \mathrm{eV}(963.7 \pm 1.2 \AA)$, which agrees with the value obtained by Samson and Cairns. The ionization potential of $\mathrm{Ar}_{2}$ was found to be $14.44 \pm 0.02 \mathrm{eV}(852.7 \pm 1.2 \AA)$. Using the known ground state dissociation energies of $\mathrm{Kr}_{2}$ and $\mathrm{Ar}_{2}$, the dissociation energy of $\mathrm{Kr}_{2}^{+}, \mathrm{B}_{0}\left(\mathrm{Kr}_{2}^{+}\right)$, is deduced to be $1.12 \pm 0.02 \mathrm{eV}$ and that for $\operatorname{Ar}_{2}^{+}, \mathrm{O}_{0}\left(A r_{2}^{+}\right)$, is $1.31 \pm 0.02 \mathrm{eV}$. The photoion yield curves of $\mathrm{Kr}_{2}$ and $\mathrm{Ar}_{2}$ are compared with that of $\mathrm{Xe}_{2}$. Prominent autoionization structure was observed to correspond to Rydberg molecular states which are derived from the combination of a normal and an excited atom in the $4 p^{5} n s$ (or $4 p^{5} n d$ ) configuration for $k r$ and $3 p^{5} n s$ (or $3 p^{5} n d$ ) configuration for Ar.

* Submitted to Journal of Chemical Physics for publication. 


\section{INTRODUCTION}

The existence of stable $\mathrm{Ar}_{2}$ molecules has long been postulated to account for the negative second virial coefficient of Ar at low temperatures. Recently, Milne and Greene' as well as many other workers have observed the formation of argon dimers and polymers in supersonic gas jets of Ar. In a mass spectrometric investigation, Leckenby et. al. ${ }^{2}$ also observed strong evidence for dimers such as $\mathrm{Ar}_{2}, \mathrm{Xe}_{2}$, etc. Reliable information on the interaction potentials for the rare gas symmetric pairs has become available in recent years from molecular beam differential and total cross sections measurements, ${ }^{3}$ careful analysis of transport phenomena and macroscopic properties, ${ }^{4}$ and high resolution vacuum UV absorption spectra. ${ }^{5}$ The high resolution vacuum ultraviolet absorption spectra of $\mathrm{Kr}_{2}$ and $\mathrm{Ar}_{2}$ obtained by Tanaka and Yoskino ${ }^{5}$ not only allowed them to locate many vibrational energy levels of ground states but also enabled them to identify many excited electronic states of $\mathrm{Ar}_{2}$ and $\mathrm{Kr}_{2}$. However, to the authors' knowledge, careful ionization studies of the rare gas van der Haals molecules either by electron or photon impact, have not been carried out.

The lower bound of the dissociation energles of the rare gas molecular ions $\left(R_{2}^{+}\right)$have been estimated from the appearance potentiais of the electron impact induced associative ionization process. ${ }^{6-10}$ The first photoionization study of the formation of the $\mathrm{Ar}_{2}^{+}, \mathrm{Kr}_{2}^{+}$and $\mathrm{Xe}_{2}^{+}$ rare gas molecular ions was carried out by Huffman and Katayama. ${ }^{11}$ The ions were produced mainly by an associative collision process between an electronically excited atom and a ground state atom, which results in 
the formation of a diatomic ion and an electron. Samson and Cairns ${ }^{12}$ also have obtained the ionization potentials of $\mathrm{Kr}_{2}$ and $\mathrm{Xe}_{2}$ from their photoionization experiment with a high pressure gas cell. Due to the interference stemming from secondary processes they were unable to identify and examine the photoionization of the rare gas van der Waals molecules alone. In order to investigate the photoionization mechanism of van der Waals dimers, we have prepared these molecules by the supersonic expansion of the corresponding monomer at high pressure through a small orifice. By combining the molecular beam technique with photoionization mass spectrometry, we carried out the first photoionization study of $\mathrm{Xe}_{2}{ }^{13}$ and demonstrated that the direct ionization mechanism of $X_{2}$ in the beam is quite different from associative ionization process observed by Hoffman and Katayama in the gas cell. Nevertheless, since the van der Waals well depth decreases from 24 meV in $\mathrm{Xe}_{2}{ }^{3}$ to $17 \mathrm{meV}$ for $\mathrm{Kr}_{2}{ }^{3}$ and $12 \mathrm{meV}$ for $\mathrm{Ar}_{2}{ }^{4}$ it would not be unreasonable to expect a corresponding gradual change in the photoionization mechanism. In this report, we present the first phototonization study of the $\mathrm{Kr}_{2}$ and $\mathrm{Ar}_{2}$ van der Waals molecules.

\section{EXPERIMENTAL}

The experimental apparatus, arrangement, and procedure are the same as previously described. ${ }^{13,14}$ The grating used was 5.21 : 1t:h $\mathrm{MgF}_{2}$ and had $1200 \mathrm{lines} / \mathrm{mm}$. The reciprocal dispersion is $8.3 \AA / \mathrm{mm}$. With a $100 \mu$ entrance and a $300 \mu$ exit slit, the resolution achieved is about 
1.2A FWHM. The light source was the helium Hopfield continuum. 15 During this experiment, the discharge lamp was operated by a high power pulser at a repetition rate of $100 \mathrm{KHz}$ and a pulse width of $\sim 0.5 \mu \mathrm{sec}$. The photon output at the exit slit of the monochromator was about $10^{10}$ photon/sec $\AA$ at $800 \AA$ as measured by a nickel photoelectric cell. At the ionization threshold region of $\mathrm{kr}_{2}$, which is around $960 \AA$, the hydrogen many-line pseudocontinuum was used as the light source. This avoided the interference of the high energy photons of the helium Hopfield continuum coming from the second-order diffraction of the grating.

The $\mathrm{Kr}_{2}$ and $\mathrm{Ar}_{2}$ van der Waals molecules were prepared by supersonic expansion through a nozzle with $0.127 \mathrm{~mm}$ diameter at a stautition pressure of 500 torr. The mixed beam of atoms and dimers formed by adiabatic cooling then intersected the dispersed vacuum ultraviolet photons at a distance of approximately $7.5 \mathrm{~cm}$ from the nozzle. With two stages of differential pumping, the main chamber pressure was maintained at $5 \times 10^{-7}$ torr during the experiment. The total number density of the beam at the collision center which contained a small fraction of dimers was estimated with an electron bombardment type fonizer to be about $10^{12}$ atom/cc. The photon detector was a sodium salicylate coated photomultiplier. Data were taken at a wavelength interval of $1 \AA$. In view of the strong pressure dependence of the concentration of the dimers relative to the monomers in the expansion, the nozzle stagnation pressure was well regulated. The photolonization efficiency of $\mathrm{Kr}_{2}\left(\mathrm{Ar}_{2}\right)$ at $870 \AA(780 \AA)$ was arbitrarily chosen as a 
reference point with which to monitor the fluctuation of the intensity of both the $\mathrm{Kr}_{2}$ and $\mathrm{Ar}_{2}$ molecules and the vacuum UV photons. For every $20 \AA$, the monochromator was scanned back to the reference point and the photoionization efficiency was recorded. It was found that the intensity of the $\mathrm{Kr}_{2}$ (or $\mathrm{Ar}_{2}$ ) dimer stayed constant within $3 \%$. The phototon yield curves thus obtained have been normalized to account for the beam fluctuations. The average counting rates at the maximum of the autoionization peaks were about $25 \mathrm{ct} / \mathrm{sec}$ for $\mathrm{Kr}_{2}$ and $15 \mathrm{ct} / \mathrm{sec}$ for $\mathrm{Ar}_{2}$. Generally, counts were collected for $200 \mathrm{sec}$ for $\mathrm{Ar}_{2}$ and $100 \mathrm{sec}$ for $\mathrm{Kr}_{2}$. However, near the ionization threshold where the photoionization cross sections were low, counts were accumulated for as long as $800 \mathrm{sec}$.

\section{RESULTS AND DISCUSSION}

Assuming the photoionization cross section of the dimer to be twice that of the atom, the variation of the concentration of the $\mathrm{Kr}_{2}$ $\left(A r_{2}\right)$ dimers relative to that of $\mathrm{Kr}(\mathrm{Ar})$ atom monitored at $870 \AA(780 \AA)$ were plotted against the nozzle stagnation pressure as shown in Fig. 1. In a static reservoir of a particular gas, the concentration of the dimers relative to the monomers as predicted by the statistical model ${ }^{2 b}$ is linearly proportional to the pressure $\left(P_{0}\right)$. However, with the expansion arrangement and condition of this work. it was found that the percentage concentration of $k r_{2}$ relative to $k r$ atoms, $\alpha\left(k r_{2}\right)$, is approximately proportional to $P_{0}^{3}$, whereas $\alpha\left(A_{2}\right)$ is only proportional to $P_{0}^{7.5}$. It is interesting to note that, by assuming a Lennard-Jones 
(6-12) potential, Sogryn and Hirschfelder ${ }^{16}$ have calculated the $\mathrm{Ar}_{2}$ dimer concentration at S.T.P. to be $1 \times 10^{-3}$. A value of $3 \times 10^{-3}$ was obtained from our experiment wher $\mathrm{Ar}$ is expanded from 1 atmosphere pressure.

The photoionization efficiency curves of $\mathrm{Kr}_{2}$ and $\mathrm{Ar}_{2}$, together with that ${ }^{13}$ of $\mathrm{Xe}_{2}$ are shown in Fig. 2. The phototonization thresholds of $\mathrm{Xe}, \mathrm{Kr}$, and $\mathrm{Ar}$ are also plotted for comparison. The graphs are plotted in energy scale $\left(\mathrm{cm}^{-1}\right)$ and are shifted such that the ionization potentials of the atoms fall in a line. The Franck-Condon factor for direct fonization is expected to be small for this system. The thresholds are not abrupt, and the curves rise very slowly until the strong autoionization structure sets in. Within the sensitivity of our photoionization mass spectrometer system, which can detect a signal as low as 0.1 count/sec (with $800 \mathrm{sec}$ counting time), the ionization potential for $\mathrm{Kr}_{2}$ is found to be $963.7 \pm 1.2 \AA(12.86 \pm 0.015 \mathrm{eV})$ and that for $\mathrm{Ar}_{2}$ is $852.7 \pm 1.2 \AA(14.44 \pm 0.02 \mathrm{eV})$. Using the known dissociation energies of the ground state $\mathrm{Kr}_{2}$ and $\mathrm{Ar}_{2}$ van der Waals dimers, ${ }^{3,4}$ the dissociation energies, $\mathrm{D}_{0}$, of $\mathrm{Kr}_{2}^{+}$and $\mathrm{Ar}_{2}^{+}$are deduced to be $1.12 \pm 0.02 \mathrm{eV}$ and 1.31 $\pm 0.02 \mathrm{eV}$ respectively. The values of $\mathrm{D}_{0}\left(\mathrm{Xe}_{2}^{+}\right), \mathrm{D}_{0}\left(\mathrm{Kr}_{2}^{+}\right)$and $\mathrm{D}_{0}\left(\mathrm{Ar}_{2}^{+}\right)$ derived from other methods are also listed in Table I for comparison with the results of this work. The values for $D_{0}\left(X_{2}^{+}\right)$and $D_{0}\left(\mathrm{Kr}_{2}^{+}\right)$ obtained by Samson and Cairns are in good agreement with our determinations. For $D_{0}\left(\mathrm{Ar}_{2}^{+}\right)$, a value as high as $2 \mathrm{eV}$ deduced from the high pressure mass spectrometric work of Kebarle, et. al. ${ }^{18}$ but this result appears to be too high. Recently, elastic differential scattering measurements have 
been performed on $\mathrm{Ar}^{+}+\mathrm{Ar}$ and $\mathrm{Xe}^{+}+$Xe at low energy $\left(\mathrm{E}_{\mathrm{c} . \mathrm{m} .}=5-25 \mathrm{eV}\right.$ ) by Lorentz and co-workers. ${ }^{17}$ Their data are found to be consistent with a potential well depth of $1.25 \mathrm{eV}$ for $\mathrm{Ar}_{2}^{+}$and $0.97 \mathrm{eV}$ for $\mathrm{Xe}_{2}^{+}$. In the vacuum ultraviolet absorption spectrum of $\mathrm{Ar}_{2}$, a diffuse band which might correspond to the formation of $\mathrm{Ar}_{2}^{+}$was observed starting at $870 \AA$. Consequently, an extremely careful scan in the wavelength region from 870 to $845 \AA$ was conducted before I.P. $\left(A r_{2}\right)$ of $852.7 \pm 1.2 \AA$ was obtained. One of the mair: purposes of this study was to investigate the photoionization mechanism for this class of van der Waals complex. By examining the photoion yield curves of the rare gas dimers $\left(\mathrm{Xe}_{2}, \mathrm{Kr}_{2}\right.$ and $\mathrm{Ar}_{2}$ ), we can conclude that autoionization is the predominant process. Autoionization essentially consists of two discrete steps. The first one is the excitation of the dimer $R_{2}$ to a resonant molecular state, and the second is the interaction between the excited Rydberg electron and the ion core which results in the ejection of the electron and formation of $R_{2}^{+}$in a discrete ionic level. The resonant molecular levels of $R_{2}$ in this energy region can only be derived from the combination of one normal ground $s$ tate $\left({ }^{\prime} s_{0}\right)$ and one excited atomic level. For the electronic states of the rare gas dimer $R_{2}$. Hund-Mulliken's case (c) applies. The ground state of $R_{2}$ has a closed electronic shell, and thus is a $\mathrm{O}_{\mathrm{g}}^{+}$state. By the selection rules of case $(r)$, transitions from $0_{g}^{+}$to $0_{u}^{+}$or $l_{u}$ excited molecular states are the only ones which are dipole allowed.

All the excited atomic states (except those with $\mathrm{J}=0$ ) can couple with the ground state rare gas atom $\left(n p^{6} l_{S_{0}}\right.$ ) (where $n=5$ for $X e, n=4$ 
for $\mathrm{Kr}$ and $\mathrm{n}=3$ for $\mathrm{Ar}$ ) to give an $\mathrm{O}_{u}^{+}$and/or an $1_{u}$ molecular state. In other words, disregarding the second step in the autoionization process, all the excited atomic levels which have an energy higher than the dissociation energy of the ground state $R_{2}^{+}$are available for autoionization. However, if we compare the positions of the peaks and the excited atomic levels, we find a very good correlation between the Rydberg atomic level dervied from $4 p^{5}\left({ }^{2} p_{1 / 2,3 / 2}\right)$ ns (or nd) $\left(3 p^{5}\left({ }^{2} p_{1 / 2,3 / 2}\right)\right.$ ns (or nd)) for $\mathrm{Kr}$ (Ar) and the autoionization peaks as shown in Fig. 2(b) and $2(c)$. The Rydberg atomic levels derived from $5 p^{5}\left({ }^{2} P_{1 / 2}, 3 / 2\right)$ ns (and nd) are also plotted in Fig. 2(a) for comparison. The correlation is not very obvious in the case of $\mathrm{Xe}_{2}$.

The dissociation energies of $\mathrm{Kr}_{2}(17 \mathrm{meV})$ and $\mathrm{Ar}_{2}(12 \mathrm{meV})$ are smaller than that of $\mathrm{Xe}_{2}(24 \mathrm{meV})$. Hence for $\mathrm{Kr}_{2}$ and $\mathrm{Ar}_{2}$, one atom feels a small perturbation from its partner. When a photon comes in, it essentially sees two separate atoms. The excitation of one of them will follow the parity selection rule $\Delta 1= \pm 1$, which is a well defined property of a free atom with any number of electrons and with any kind of coupling. In other words, the molecular Rydbery orbital is essentially a tightly bound atomic orbital associated entirely with the excited atom. With the atoms of the dimer in the ground state $\left(1_{s_{0}}\right)$, the selection rule $\Delta J=+1$, which is valid for free rare gas atoms, no longer holds. All the atomic levels with different $J$ values (except $J=0$ ) which are derived from $5 p^{5}\left({ }^{2} p_{1 / 2}, 3 / 2\right)$ ns (and nd) for $\mathrm{xe}, 4 p^{5}\left({ }^{2} \mathrm{p}_{1 / 2}, 3 / 2\right)$ ns (and $n d$ ) for $\mathrm{Kr}$, and $3 p^{5}\left({ }^{2} \mathrm{p}_{1 / 2}, 3 / 2\right)$ ns (and nd) for Ar by $\mathrm{Jl}$ coupling scheme ${ }^{19}$ are expected to be allowed tran- 
sitions as a result of the perturbation by its partner. Nevertheless, the dimer is just like a persistent collision complex, and the probability of vibrationally induced autoionization will be large. In fact, the autoionization lifetime will be much shorter than the raciative lifetime. This seems to explain well the observed autoionization structure for $\mathrm{Kr}_{2}$ and $\mathrm{Ar}_{2}$ shown in Fig. $2(\mathrm{~b})$ and $2(\mathrm{c})$. However, this by no means excludes the autoionization of the Rydberg states which are derived from an excited $\mathrm{Kr}(\mathrm{Ar})$ atom with the configuration $4 p^{5}\left({ }^{2} \mathrm{P} 1 / 2,3 / 2\right) \mathrm{np}$ (or $n f$ ) $3 p^{5}\left({ }^{2} p_{1 / 2,3 / 2}\right) n p(o r n f)$ ) and a normal ground state $\mathrm{Kr}$ (Ar) atom. In fact, it is possibly the autoionization of these levels that gives rise to a finite photoionization efficiency at the onset of the rare gas dimers.

\section{ACKNOWLEDGEMENT}

This work was done with support from the U. S. Energy Research and Development Administration. 


\section{REFERENCES}

1a. F. T. Greene and T. A. Milne, J. Chem. Phys., 39, 3150 (1963).

b. T. A. Milne and F. T. Greene, J. Chem. Phys., 47, 4095 (1967).

2a. R. E. Leckenby, E. J. Robbins and P. A. Trevalion, Proc. Roy. Soc. A, 280, 409 (1964).

b. R. E. Leckenby and E. J. Robbins, Proc. Roy. Soc. London, Ser. A, 291, 389 (1966).

3. J. M. Parson, P. E. Siska and Y. T. Lee, J. Chem. Phys., 56, 1511 (1972).

4. J. A. Baker, K. O. Watts, J. K. Lee, T. P. Schafer and Y. T. Lee, J. Chers. Phys., 61, 308 (1974).

5a. Y. Tanaka, K. Yoshino and D. E. Freeman, J. Chem. Phys., 59, 5160 (1973).

b. Y. Tanaka and K. Yoshino, J. Chem. Phys., 53, 2012 (1970).

6. J. A. Hornbeck and J. P. Molnar, Phys. Rev., 84, 621 (1951).

7. W. Kaul and R. Fuchs, 2. Naturforch, 152, 326 (1960).

8. W. Kaul and R. Taubert, 2. Naturforch, 17a, 88 (1962).

9. M. 5. Munson, J. L. Frankl in and F. H. Field, J. Phys. Chem., 67, 1542 (1963).

10. C. E. Mel ton and W. H. Hamill, J. Chem. Phys., 41. 1469 (1964).

11. R. E. Huffman and D. H. Katayama, J. Chem. Phy5., 45, 138 (1966).

12. J. A. R. Samson and R. B. Cairns, J. Opt. Soc. Am., 56, 1140 (1966).

13. C. Y. Ng, D. J. Trevor, B. H. Mahan and Y. T. Lee, J. Chem. Phys., to be published.

14. C. Y. Ng, B. H. Mahan and Y. T. Lee, J. Chem. Phys., to be published. 
15a. J. J. Hopfield, Phys. Rev., 35, 1133 (1930).

b. J. J. Hopfield, Phys. Rev., 36, 784 (1930).

c. J. J. Hopfleld, Astrophys. J., 72,133 (1930).

16. D. E. Stogryn and J. O. Hirschfelder, J. Chem. Pinys., 31, 1531 (1959).

17. D. C. Lorentz R. E. 01 son and G. M. Conkl in, Chem. Phys. Let+.., 20, $589(1973)$.

18. P. Kebarle, R. M. Haynes and S. K. Searles, J. Chem. Phys., 47, 1684 (1967).

19. C. E. Moore, Atomic Energy Levels, NBS Cir. 467, Vol I (1949), II (1952), III (1958). 


\section{FIGURE CAPTIONS}

Fig. 1. Variation of the $\mathrm{Kr}_{2}$ and $\mathrm{Ar}_{2}$ rare gas dimers relative to the corresponding monomer versus nozzle stagnation pressure.

- experimental points for $\mathrm{Kr}_{2}$ obtained at $870 \mathrm{~A}$

- - approximate fit, $\%$ concentration $\alpha\left(k r_{2}\right)=1.81 \times 10^{-3}$ $\left[\mathrm{P}_{0} \cdot \mathrm{D}_{0}\right]^{3}$

O experimental points for $\mathrm{Ar}_{2}$ obtained at $780 \AA$

- approximate fit. $\alpha\left(A r_{2}\right)=1.01 \times 10^{-2}\left(P_{0} \cdot D_{0}\right)^{1.5}$ where $P_{0}$ (torr) is the nozzle stagnation pressure and $D_{0}(=0.0127 \mathrm{~cm})$, the nozzle diameter.

Fig. 2. (a) Photoionization efficiency curve of $X_{2}$ in the energy range from $89,000 \mathrm{~cm}^{-1}$ to $103,000 \mathrm{~cm}^{-1}$.

(b) Photolonization efficiency curv of $\mathrm{Kr}_{2}$ in the energy range from $103,000 \mathrm{~cm}^{-1}$ to $118,000 \mathrm{~cm}^{-1}$.

(c) Photoionization efficiency curve of $\mathrm{Ar}_{2}$ in the energy range from $117,000 \mathrm{~cm}^{-1}$ to $132,000 \mathrm{~cm}^{-1}$. 
Table I. Rare-gas Ion Dfssociation Energfes $D_{0}$

Electron Impact

Appearance Poténtials

Theoretical

Miscellaneous

Photofonization

\begin{tabular}{|c|c|c|c|c|c|c|c|c|c|c|c|c|c|}
\hline : & (a) & (b) & (c) & (d) & (e) & $(f)$ & (g) & (h) & (i) & (j) & $(k)$ & (i) & (m) \\
\hline $\mathrm{Ar}_{2}^{+}$ & $\begin{array}{l}1.08 \\
\pm 0.1^{1}\end{array}$ & 0.66 & 0.66 & $\begin{array}{r}0.84 \\
\pm 0.02\end{array}$ & 0.66 & 1.25 & 1.21 & 2 & 0.0035 & 1.25 & $\begin{array}{r}1.049 \\
\pm 0.009\end{array}$ & & $\begin{array}{r}1.31 \\
\pm 0.02\end{array}$ \\
\hline $\mathrm{Kr}_{2}^{+}$ & $\begin{aligned} & 1.00 \\
= & 0.1\end{aligned}$ & 0.8 & & $\begin{array}{c}0.8 \\
\pm C .02\end{array}$ & & & 0.92 & & & & $\begin{array}{r}0.995 \\
\pm 0.007\end{array}$ & 1.13 & $\begin{array}{c}1.12 \\
\pm 0.016\end{array}$ \\
\hline $\mathrm{Xe}_{2}^{+}$ & $\begin{array}{r}0.97 \\
\pm 0.07\end{array}$ & & & & 0.5 & & 0.65 & & & 0.97 & $\begin{array}{r}0.968 \\
\pm 0.005\end{array}$ & $\begin{array}{r}0.99 \\
=0.02\end{array}$ & $\begin{array}{r}1.03 \\
=0.01\end{array}$ \\
\hline 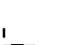 & & & . & & & & & & & & & & \\
\hline
\end{tabular}

(a) Reference ?

(b) Reference 4

(c) Reference 5

(d) Reference 8

(e) Reference 6

(f) :. L. Gllbert and A. C. Wahl (unpublished SCF calculation)

(g) R. S. Mulliken, 52, 5170 (1970)

(h) Reference 18

(i) E. A. Mason and J. T. Vanderstice, J. Chem. Phys., 36, 1103 (1962).

(j) Reference 17

if Reference 9

$\because$ zeference :0

(ii) Tis work. 


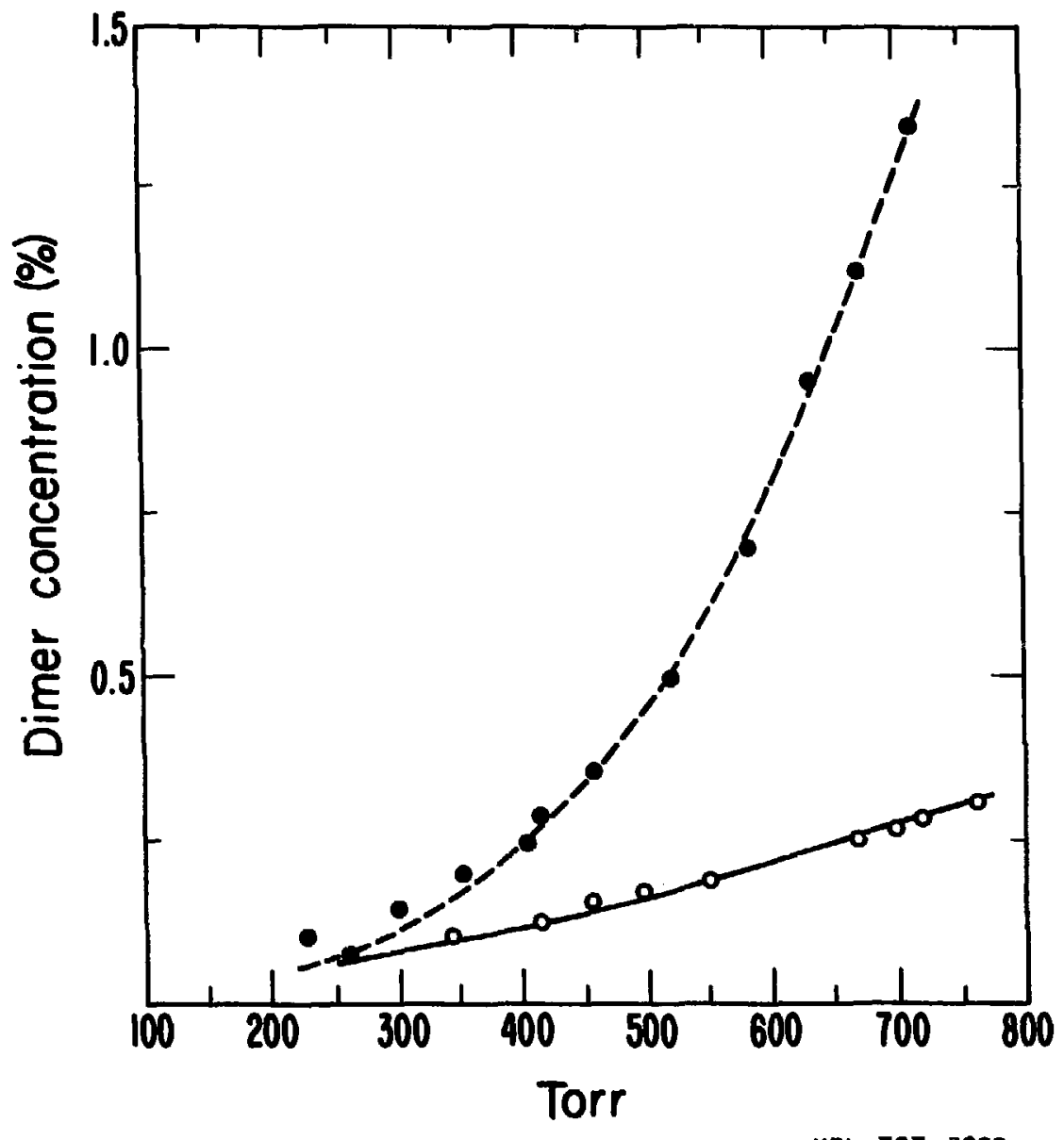

Fig. 1

XBL 767-3200 


$$
2 \cdot 6 ! 4
$$

onst dn $78 x$

$$
1-\text { - }{ }^{2}-01 x
$$

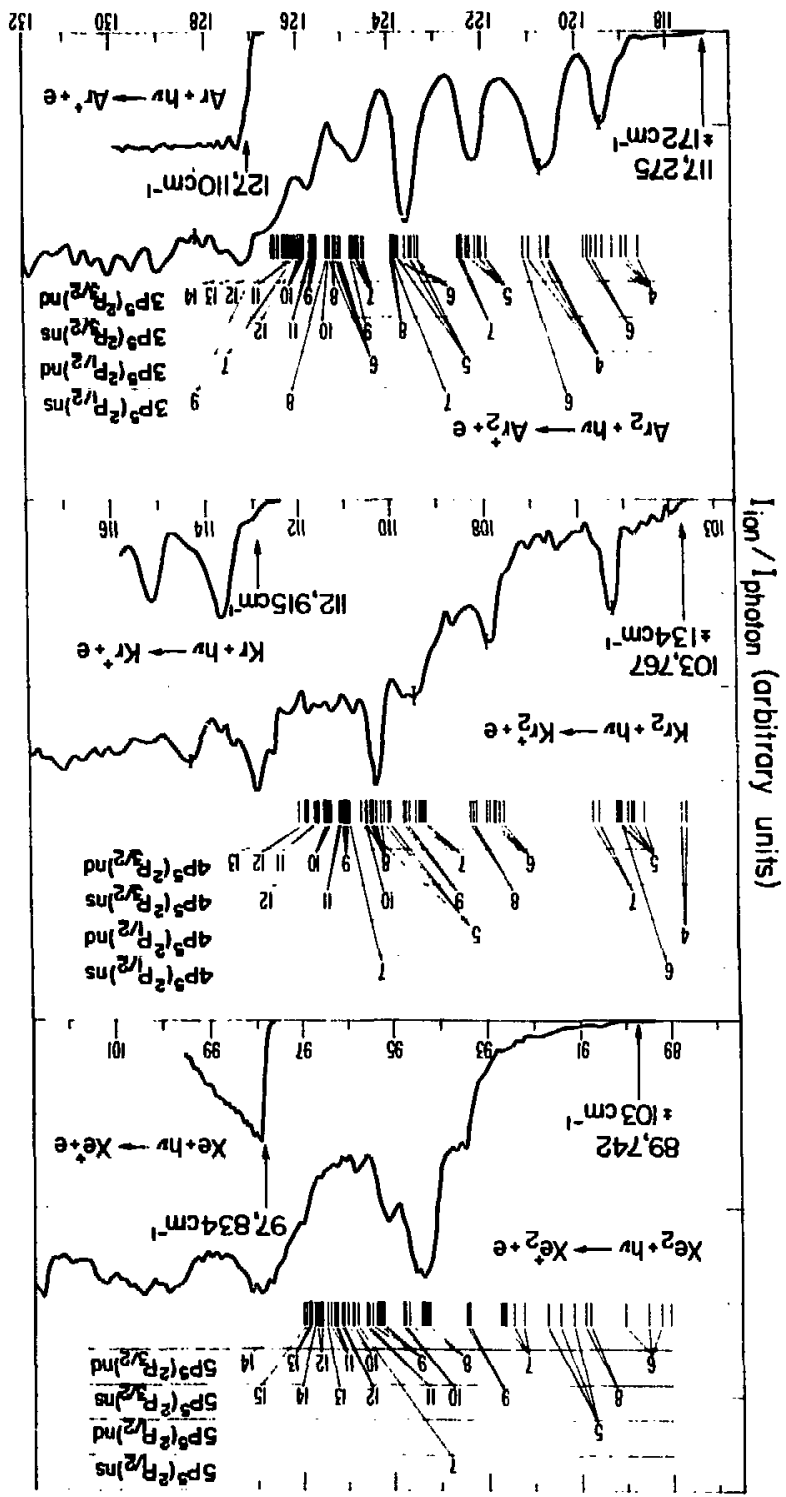


VI. PhOTOIONIZATION STUdY OF ICI AND THE ArICl VAN DER waALS MOLECULE

\section{ABSTRACT}

Photoionization effictency curves of ICl and the ArICl van der Waals dimer were obtained with the molecular beam technique in the wavelength range from $1140 \AA$ to $1245 \AA$ (9.958 eV $-10.375 \mathrm{eV}$ ) and from $1190 \AA$ to $1250 \AA$ (9.919 eV - $10.418 \mathrm{eV})$ respectively. The ionization potential of ICl from the ground electronic state $\left(\Sigma^{+}, v^{\prime \prime}=0\right)$ to the $\mathrm{ICl}^{+}$ground molecular ionic state $\left({ }^{2} \Pi_{3 / 2 g}, v^{\prime}=0\right)$, was found to be $10.069 \pm 0.003 \mathrm{eV}$ which is in agreement with the value obtained by Dibeler et. al. The ionization potential corresponding to the ionization of $\operatorname{ICl}\left(\Sigma^{+}, v^{\prime \prime}=0\right)$ to $\operatorname{ICl}^{+}\left({ }^{2} \Pi_{1 / 2 g^{*}} v^{\prime}=0\right)$ was found to be $10.699 \pm 0.003 \mathrm{eV}$. By analyzing the photoion yield curve of $\mathrm{ICl}$, the vibrational quantum for the $\mathrm{ICI}^{+}\left({ }^{2} \pi_{3 / 2 g}\right)$ was deduced to be $428 \pm 20$ $\mathrm{Cm}^{-1}$. The excitation efficiency leading to lon-pair formation (i.e. $\mathrm{ICl}+\mathrm{hv}+\mathrm{I}^{+}+\mathrm{Cl}^{-}$) was also observed for ICl by monitoring the $\mathrm{I}^{+}$ atomic ion. Using the known electron affinity for $\left.\mathrm{Cl}^{2} \mathrm{P}_{3 / 2}\right)$ atom, the dissociation energy for ICl is deduced to be $2.140 \pm 0.003 \mathrm{eV}$. For ArIC1, the ionization potential was observed to be $9.960 \pm 0.003$ eV which is $0.109 \pm 0.003 \mathrm{eV}$ lower than the I.P. of ICI. The photofonization mechanism of the ArICl van der Waals molecule is discussed. 
INTRODUCTION

In the studies of the ionization processes of atoms and molecules, the photoionization technique offers many advantages over the electron impact method. First, all the difficulties that contribute to the instability and poor resolution of the electron impact experiments such as sample pyrolysis due to hot filament, the presence of space charge due to the electron beam and the effects of charging up the surfaces of the ion optics, are avoided in the photoionization method. Second, the threshold law for photoionization is much more favorable for investigating the ionization potentials of atoms and molecules. The typical instrumental resolution for photoionization is 1 - 10 meV. However, due to the rotational (and vibrational) temperature of the molecules which is about $30 \mathrm{meV}$ at room temperature, this value of the resolution essentially is unattainable unless the sample gas is cooled down to the corresponding temperature. The cooling of the gas in a gas cell to e.g. $77^{\circ} \mathrm{K}(\sim \mathrm{meV})$ in a conventional photoionization experiment can only be feasible for noncondensible gases such as $\mathrm{H}_{2}$. For condensible gases, one of the methods of overcoming this problem is to use the superson ic beam technique to introduce the target gases to the ionization region. In an isentropic expansion, individual molecules can be easily supercooled to well below $20^{\circ} \mathrm{K}$. This has been demonstrated by the high resolution obtained for the photoionization efficiency (P.E.) curves of $\mathrm{NO}^{\top} \mathrm{C}_{2} \mathrm{H}_{2}$ and $\mathrm{CH}_{3} \mathrm{I}^{2}$ in photoionization studies which combined molecular beam techniques and photoionization mass spectrometry. As a result of adiabatic cooling, a small percentage 
of dimer and polymer will be formed during the expansion. This provides a unique way of synthesizing van der Waals molecules for photoionization studies. Recently, the photoion-yield curves of diatomic halogen and interhalogen molecules have been obtained by Dibeler et. al. ${ }^{3}$ Recognizing the thermal population of the excited vibrational states at room temperature, they have applied room temperature and cooled-gas photoionization to distinguish the hot band effect (i.e., the apparent lowing of a threshold value due to the ionization of vibrationally-excited molecules to form ground-state ions) and have identified the adiabatic ionization thresholds for the diatomic homonuclear halogen molecules and for the interhalogens. Iodine monochloride was chosen for this photoionization experiment as an example to investigate the influence of adiabatic cooling on the hot band effect. The photoionization study of the ArICl van der Waals molecule also permits us to examine the photoionization mechanism of this class of weak complexes.

\section{EXPERIMENTAL}

The experimental apparatus, arrangement, and procedure is the same as previously described. ${ }^{1}$ Iodine monochloride (ICI) was the practlcal grade obtained from Matheson, Coleman and Bell and was used without further purification. At room temperature, ICl has a vapor pressure of approximately 30 torr. In this experiment ICl vapor was premixed with Ar and then the mixture was introduced into the main chamber as the target gases by expansion through a $0.12 \mathrm{~mm}$ diameter pyrex nozzle 
at a total stagnation pressure of 450 torr. The light source was the hydrogen many-line pseudocontinuum. The grating used was coated with $\mathrm{MgF}_{2}$ and had $1200 \mathrm{lines} / \mathrm{mm}$. The reciprocal dispersion was $8.3 \AA / \mathrm{mm}$. Using the $100 \mu$ entrance and exit slit, the resolution achieved was $0.83 \AA$ ( $-6.5 \mathrm{meV})$ at $1240 \AA$. Data were collected at a wavelength interval of $0.5 \AA$. The dispersed light coming out from the exit slit was monitored by a sodium salicylate coated photomultiplier. Wavelength calibration was accomplished by using the known emission lines of the hydrogen atom and molecule. The photon signal has been corrected for the background of the stray photons from the monochromator. With two stages of differential pumping, the main chamber pressure was always below $5 \times 10^{-7}$ torr during the experiment. The counting rates varied from $100 \mathrm{ct} / \mathrm{sec}$ to $900 \mathrm{ct} / \mathrm{sec}$ for $\mathrm{ICl}^{+}$. For $\mathrm{I}^{+}$, the average counting rate at the major autoionization peaks was about $150 \mathrm{ct} / \mathrm{sec}$. Counts were accumulated for $100 \mathrm{sec}$ and $150 \mathrm{sec}$ for $\mathrm{ICl}^{+}$and $\mathrm{I}^{+}$respectively. The intensity of ArICI relative to that of ICl as probed at $1215.7 \AA$ (Lyman $\alpha$ ) was about $1.8 \%$. Thus, in the photoionization experiment of $\mathrm{ArICl}$, each point was counted for $350 \mathrm{sec}$.

\section{RESULTS AND DISCUSSION}

\section{A. Iodine-Monochloride (ICI)}

The photoionization efficiency curve obtained is shown in Fig. 1. The structure of the P.E. curve is similar to that obtained by Dibeler 
et. al. ${ }^{3 a}$ The ground electronic state of ICl is a $\Sigma^{+}$state. The first ionization process involves removing an electron from an antibonding $\pi^{*}$ orbital and leaving the $\mathrm{ICl}^{+}$molecular ion in the ground ${ }^{2} \Pi_{3 / 2 g}$ state. The sharp onset at $10.069 \pm 0.003 \mathrm{eV}$ as observed from the P.E. curve corresponds to the adiabatic ionization potential of $\mathrm{ICl}$ from the ground state $\left({ }^{l} \Sigma^{+}, v^{\prime \prime}=0\right)$ to the ground ionic state $\left({ }^{2} \Pi_{3 / 2 g}, v^{\prime}=0\right)$. The I.P. which corresponds to the excited molecular ionic state ${ }^{2} \pi_{1 / 2 g}$ was found to be $10.699 \pm 0.003 \mathrm{eV}$.

The small hump before the onset in the P.E. curve is attributed to the hot band effect. The vibrational quantum of $\left.1 \mathrm{Cl}^{1} \Sigma_{\Sigma}^{+}\right)$is $384 \mathrm{~cm}^{-1}$. At room temperature, the ratio of the number of molecules in the first to the ground vibrational state assuming the Boltzman distribution is about $\mathbf{0 . 1 5}$. The peak intensity of the hump relative to that of onset peak is slightly less than $10 \%$. Thus, we conclude that with the present beam production arrangement, the vibrationally excited states of ICI do not relax efficiently. However, as judged from the onset of the ionization threshold, the relaxation of rotational energy is realized.

The P.E. curve of ICl exhibits quite regular autoionization structure which possibly consists of electronic and vibrational autoionization series. Although autofonization is the predominant ionization process for $\mathrm{ICl}$, if the Franck-Condon factors between the ground state $\operatorname{ICI}\left(I_{\Sigma}^{+}, v^{\prime \prime}=0\right)$ and the $\left.\mid \mathrm{ICl}\right]^{+}\left({ }^{2} \pi_{3 / 2 g}, v^{\prime}\right)$ molecular foric states are favorable, direct ionization st1ll would take place. By examining the (HeI) photoelectron spectrum of ICl, Cornford has been able to resolve about five vibrational states. In other words, the leading 
edges of the first five broad peaks in the P.E. curve of ICI are contributed by direct ionization. The results of the analysis of the energy separation between adjacent (leading edge) autoionization peaks is shown in Table I. The leading edges as assigned as the onsets of the corresponding vibrational states are positioned by solid lines in Fig. 1. The average of the vibrational quantum is $428 \pm 25 \mathrm{~cm}^{-1}$ which is in good agreement with that obtained by photoelectron spectroscopy. 4

The autoionization peaks (the second, the fifth, the seventh, and the ninth) have higher photoion yield than the rest, and it is therefore not unreasonable to assume that these peaks have more than one autoionization peak superimposed on each other. The trend of these peaks suggests that they belong to a electronic Rydberg series. Using the ionization potential of ICl from the ground $\Sigma_{\Sigma}+v^{\prime \prime}=0$ state to the $\mathrm{ICl}^{+}\left({ }^{2} \Pi_{1 / 2 g}, v^{\prime}=0\right)$ molecular ionic state as converging limit, several peaks were identified as members of a Rydberg series described by the Rydberg equation

$$
h \nu(e v)=10.699-\frac{13.6074}{(n-0.1)^{2}} \quad n=5,6,7,8,9,10
$$

The Rydberg series observed for ICl is very similar in structure to that observed ${ }^{2}$ for $\mathrm{CH}_{3} \mathrm{I}$. They may both result from the excitation of a nearly nonbonding electron which is localized mainly around the jodine atom.

The efficiency of the ion-pair formation process, as monitored by $\mathrm{I}^{+}$atomic ion, from the onset to $1210 \AA$ is shown in Fig. 2 . The excitation efficiency curve comprises mainly two broad peaks which 
are modulated by sharp autoionization structure. Similar structure has been obtained for $\mathrm{Br}_{2}$. The threshold of the ion-pair formation process was found to be $1380.3 \AA(8.982 \pm 0.003 \mathrm{eV})$ which corresponds to the excitation process

$$
\mathrm{ICl}\left({ }^{1} \Sigma^{+} \cdot v^{\prime \prime}=0\right)+\mathrm{hv} \longrightarrow \mathrm{I}^{+}\left({ }^{2} \mathrm{P}_{2}\right)+\mathrm{Cl}^{-}\left({ }^{1} \mathrm{~S}_{0}\right)
$$

If the bond dissociation energy for ICl and the ionization potential of the lodine atom is precisely known, we can determine the electron affinity for the $\mathrm{Cl}$ atom more accurately. On the other hand, using the ionization potential of the iodine atom $(10.457 \mathrm{eV})^{5}$ and the known electron affinity for chlorine atom, ${ }^{6} E\left(\mathrm{Cl}\left({ }^{2} \mathrm{P}_{3 / 2}\right)\right)=3.613 \pm$ $0.003 \mathrm{eV}$, we obtain from the relation

$$
8.982 \mathrm{eV}=1 . P .(1)-E(C 1)+D_{0}(I-C I)
$$

the bond dissocation energy for $I C l, D_{0}(I-C l)$, of $2.140 \mathrm{eV} \pm 0.003 \mathrm{eV}$. This is slightly less than the spectroscopic value.?

B. The ArICl van der Waals molecule.

The photoionization efficiency curve for the ArICl van der Waals molecule is shown in Fig. 3. The ionization potential of ArICl was found to be $9.960 \pm 0.003 \mathrm{eV}$, which is $0.109 \mathrm{eV}$ lower than the I.P. of ICl. The ArICl van der Waals molecules which is synthesized by isentropic expansion is likely to have a distribution of internal energy. Thus the resolution obtained for the P.E. curve of ArICl as shown in Fig. 3 is expected to be worse than that of ICI alone. By shifting the P.E. curve of ICl to lower energy and normalizing the I.P. of ICl with that of $\mathrm{ArICl}$, we found that the structures of the two spectra are very similar. 
Thus, the photoionization process of ArICl is essentially the same as that of ICl except the I.P. of ArICl is lower than that of ICI. It is interesting to note that, there is a narrow step which is found to be reproducible at the onset in the P.E. curve of ArICl, this may have related to the distribution of the internal energy for ArICl. Assuming $\varepsilon^{+}$is the dissociation energy for the $\mathrm{Ar}^{+}(\mathrm{ICl})^{+}$complex, and $E$ is that for $\mathrm{Ar}-\mathrm{ICl}$, the following relation can be derived:

$$
\text { or } \begin{aligned}
& \varepsilon^{+}+\text {I.P. }(\text { Ar-ICI) }=\varepsilon+\text { I.P. (ICI) } \\
& \varepsilon^{+}-\varepsilon=0.109 \pm 0.003 \mathrm{eV}
\end{aligned}
$$

The minimum value of $\varepsilon^{+}, \varepsilon_{\min }^{+}$, is thus equal to $0.109 \mathrm{eV}$. The vibrational quantum of $\left.\mathrm{ICI}^{l}{ }^{+} \Sigma^{+}\right)$as obtained from our experiment is $\Delta E_{v}=0.053 \mathrm{eV}$. Gue to intramolecular energy transfer, it is expected that $\mathrm{Ar}-\mathrm{ICl}^{+}\left({ }^{2} \mathrm{I}_{3 / 2 g}\right.$, $\left.v^{\prime}\right)$ will not be bound, if $\mathrm{ICl}^{+}$has vibrational energy bigger than $\varepsilon^{+}$. According to our estimates, $\varepsilon_{\text {min }}^{+}>2 \Delta E_{v}$ (where $\Delta E$ is the vibrational quantum of $\left.\mathrm{ICl}^{+}\left({ }^{2} \Pi_{3 / 2 g}\right)\right)$. That is $\operatorname{Ar}-\mathrm{ICl}^{+}\left({ }^{2} \Pi_{3 / 2 g}, v^{\prime}=0\right)$ and $\operatorname{Ar}-\mathrm{ICl}^{+}\left({ }^{2} \mathrm{II}_{3 / 2 g}, v^{\prime}=1\right)$ must be bound. 


\section{REFERENCES}

1. C. Y. Ng, B. H. Mahan and Y. T. I.ee, J. Chem. Phys., to be published.

2. C. Y. Ng, Ph.D. Thesis, The University of California, Berkeley, 1976.

3a. V. H. Dibeler, J. A. Walker, K. E. McCulloh and H. M. Rosenstock, Int. J. Mass. Spectrom. Ion. Phys., 7, 209 (1971).

b. V. H. Dibeler, J. A. Wa!ker and K. E. McCulloh, J. Chem. Phys., 53, $4414(1970)$.

c. V. H. Dibeler, J. A. Walker and K. E. McCulloh, J. Chem. Phys., 53, 4715 (1970).

4. A. B. Cornford, Ph.D. Thesis, The University of British Columbia, 1971.

5. C. E. Moore, Atomic Energy Levels, NBS Circ., 467, Vol. 3 (1958).

6. R. S. Berry and C. W. Reiman, J. Chem. Phys., 38, 1540 (1963).

7. G. Herzberg, "Molecular Spectra and Molecular Structure, I. Spectra of Diatomic Molecules," Van Nostrand Reinhold Coinpany, 2nd Ed., P.542, New York, (1950). 
Table I. The vibrational quanta of $\operatorname{ICl}^{+}\left({ }^{2} \pi_{3 / 2 g}\right)\left(\mathrm{cm}^{-1}\right)$

$\begin{array}{lllllll}\Delta v^{\prime}(a) & 0-1 & 1-2 & 2-3 & 3-4 & 4-5 & 5-6 \\ \Delta E_{v^{\prime}}{ }^{(b)} & 430 & 435 & 423 & 428 & 432 & 420\end{array}$

(a) $v^{\prime}$ is the vibrational quantum number

(b) $\Delta E_{v^{\prime}}=$ vibrational quantum between adjacent vibrational states (the uncertainty of these values is $\pm 25 \mathrm{~cm}^{-1}$ ). 


\section{FIGURE CAPTIONS}

Fig. 1. Photoionization efficiency curve of ICI in the wavelength range from $1140 \AA$ to $1245 \AA(9.958 \mathrm{eV}-10.875 \mathrm{eV})$.

Fig. 2. Excitation efficiency curve of the ion-pair formation process for $\mathrm{ICl}$ by monitoring the $\mathrm{I}^{+}$ion in the wavelength range from $1210 \AA$ to $1390 \AA(8.919 \mathrm{eV}-10.246 \mathrm{eV})$.

Fig. 3. Photoionization efficiency curve of the ArICl van der Waals molecules in the wavelength range from $1190 \AA$ to $1250 \AA$ $(9.919 \mathrm{eV}-10.418 \mathrm{eV})$. 


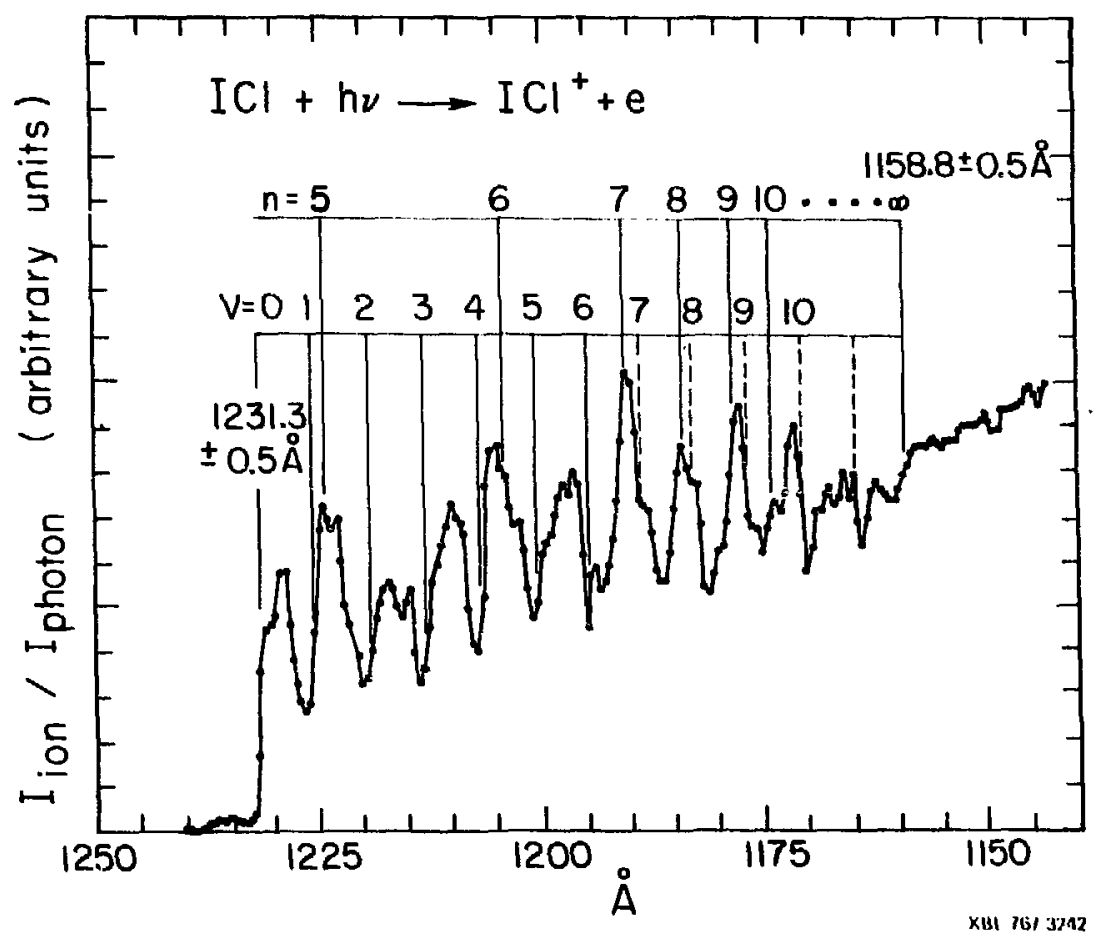

Fig. 1 


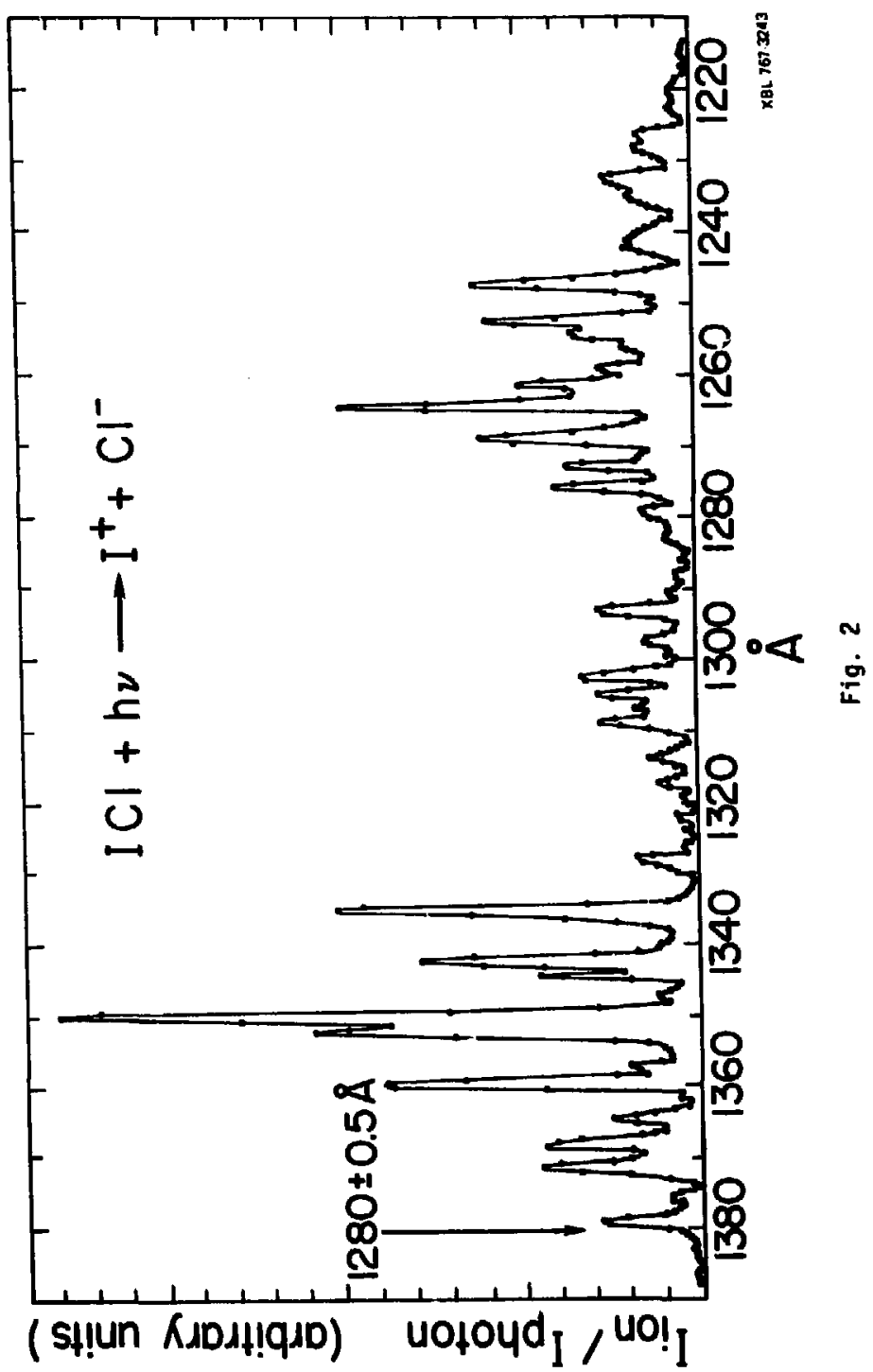




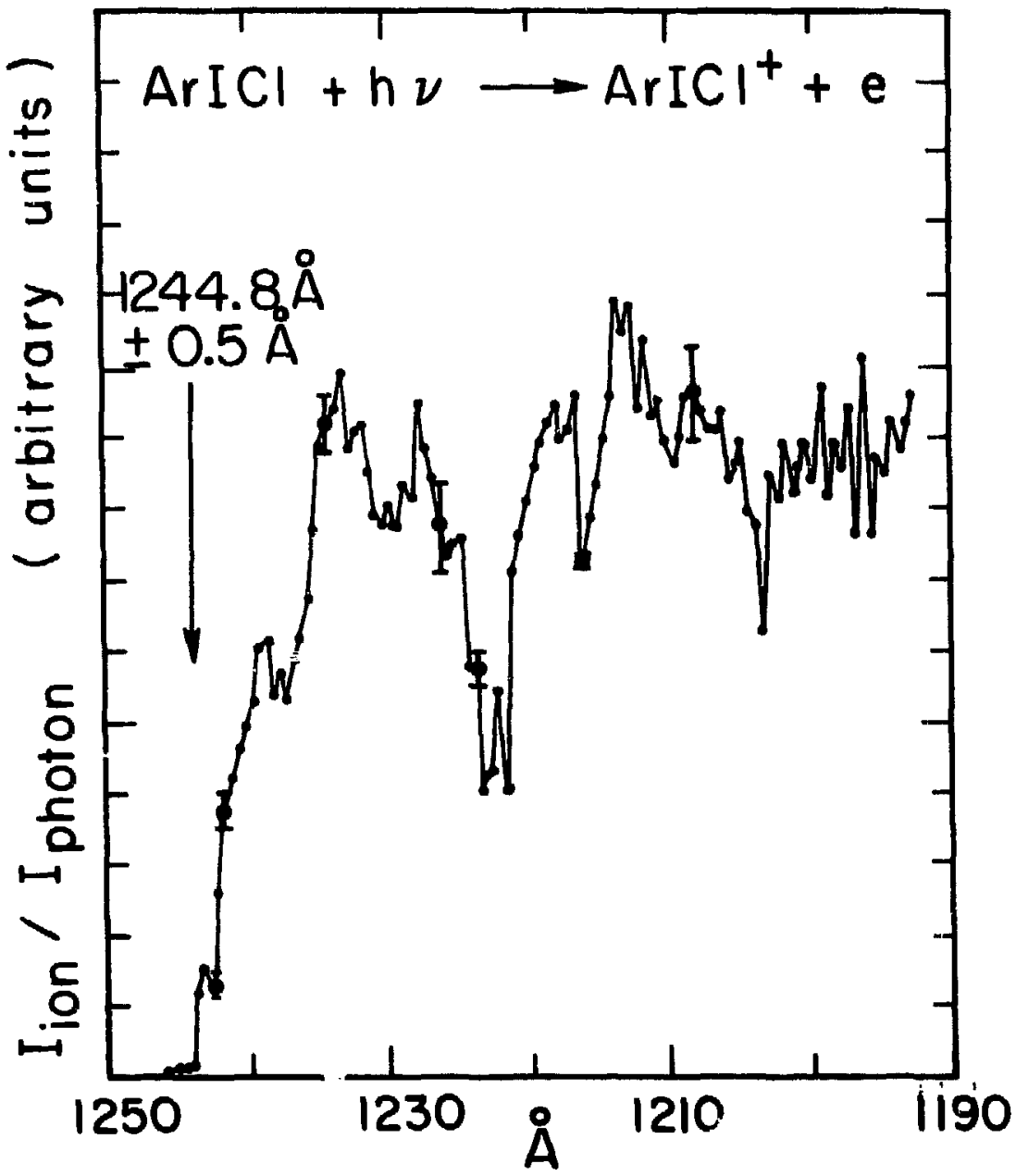

Fig. 3 
VII. PhOtOIOnIzATION StudY OF ACETYLENE $\left(\mathrm{C}_{2} \mathrm{H}_{2}\right)$ and METhYL IODIOE $\left(\mathrm{CH}_{3} \mathrm{I}\right)$ A. Photoionization Study of Acetylene $\left(\mathrm{C}_{2} \mathrm{H}_{2}\right)$

\section{INTRODUCTION}

There is a general agreement on the first fonization threshold value of acetylene as derived by photidionization studies, ${ }^{1-5}$ photoelectron spectroscopy, 6 vacuum ul traviolet absorption spectroscopy 7,8 and monoenergetic electron inpact. 9 Photefonization studtes have also shown that, the fonization of acetylene $\mathrm{C}_{2} \mathrm{H}_{2}\left({ }_{\Sigma_{9}^{*}}^{I^{*}}\right)$ to the ground wolecular tonic state $\mathrm{C}_{2} \mathrm{H}_{2}^{+}\left({ }^{2} \pi_{u}\right)$ gives rise to a step-function behavior corresponding to the ground, and first and second vibrationaliy excited states of the Ion. Recently, Dibeler et. al.. obtained a high resolution phototonization curve of $\mathrm{C}_{2} \mathrm{H}_{2}$ and resolved the weak autoionization structure which is superimposed on the steps. Their experiment was done by cooling acetylene in a low pressure gas cell to $118^{\prime} K$, which lowered the rotationsl envelope to approximately a width of $0.01 \mathrm{eV}$. In our photoionization study, acetylene was chosen to investigate the rotational relaxation through supersonic gas expansion. With the $100 \mu$ entrance and exit slits on a 1 meter monochronator, the instrumental resolution achieved will be about $0.01 \mathrm{eV}$ in the wavelength regian which corresponds to the fonization threshold of acetylene. Thus, It is expected that, if the rototion energy of acetylene is relaxed by supersonic expansion, we would obtain a phototionization efficiency curve with at least as good resolution as that employed by Dibeler. 


\section{EXPERIMENTAL}

The experimental apparatus, arrangetient and procedure were the same as previc sly described. ${ }^{10}$ The acetylene gas $199.6 \%$ minimum purity) obtained from Matheson without further purification. Acetylene was introduced into the main chamber as a target gas by supersonic expansion through a $0.127 \mathrm{~mm}$ diameter nozzle at stagnation pressure of 300 torr. The stability of the stagnation pressure was well regulated by a needle valve (Edwards high vacuum LTD). Data were taken at a wavelength interval of $0.5 \AA$. From the onset to $1040 \AA$, counts were accumulated for $40 \mathrm{sec}$. In the wavelength region $1040 \AA$ to $1020 \AA$, each point was counted for 100 sec. The average counting rate at the threshold was about $60 \mathrm{ct} / \mathrm{sec}$.

\section{RESULTS AND DISCUSSIOH}

The photoionization efficiency (P.E.) curve obtained in the wavelength region from $1020 \AA$ to $1095 \AA$ (11.322 eV - $12.155 \mathrm{eV})$ for acetylene is show in FIg. 1. As expected, the P.E. curve of acetylene compares favorably with that obtained by Dibeler. In other words, the rotational temperature in our experiment is less than $118^{\circ} \mathrm{K}$ after the expansion with our beam production system.

The ground state electronic configuration of acetylene can be expressed as: 11

$$
C_{2} H_{2}:\left(\sigma_{g} \mid S_{c}\right)^{2}\left(o_{u} \mid S_{c}\right)^{2}\left(2 \sigma_{g}\right)^{2}\left(2 \sigma_{u}\right)^{2}\left(3 \sigma_{g}\right)^{2}\left(1 \pi_{u}\right)^{4}, 1_{\Sigma}^{+}
$$


The first ion onset, which is observed to be $11.398 \pm 0.005 \mathrm{eV}$. corresponds to the energy required to remove one of the $1 \pi$ electrons and leave the molecular ion in $a^{2} \pi_{u}$ state. Wilkinson ${ }^{8}$ has obtained high resolution absorption spectra for $\mathrm{C}_{2} \mathrm{H}_{2}$ and $\mathrm{C}_{2} \mathrm{D}_{2}$ in the vacuum ultraviolet. Several electronic transitions were identified. One of these, designated $3 R$, is of a Rydberg type and evidently involves a linear upper state. The $v_{2}$ (C-C stretching frequency) vibrational mode of the $3 R$ state was found to be $0.23 \mathrm{eV}$ for $\mathrm{C}_{2} \mathrm{H}_{2}$. This value compares favorably with the vibrational intervals which have an average value of $0.21 \mathrm{eV}$ resolved from the P.E. curve of $\mathrm{C}_{2} \mathrm{H}_{2}$. On this basis, early studies suggested that the Ionization of $\mathrm{C}_{2} \mathrm{H}_{2}$ from the ground electronic state $\left(\Sigma^{+}, v^{\prime \prime}=0\right)$ to the ground molecular ionic state ${ }^{2} \pi_{u}$ involves the excitation of the $v_{2}$ (C-C stretching) moda for $\mathrm{C}_{2} \mathrm{H}_{2}^{+}$. The sumbary of ionization energies for acetylene obtained from this work is listed in Table 1 .

Beside the simple step structure as observed from the P.E. curve of acetylene, the curje also contains a series of peaks superimposed on the steps. These features correspond to weak autolonization of one or more Rydberg sertes converging to the several limits of the vibrationally excited states of $\mathrm{C}_{2} \mathrm{H}_{2}^{+}$. Two Rydberg series in the vacuum ultraviolet absorption spectrum of acetylene were identified by Price.

$$
\begin{array}{ll}
\text { hu(eV) }=11.416-\frac{13.607_{4}}{(n-0.50)^{2}} & \text { Series I } \\
h v(e V)=11.400-\frac{13.607_{4}}{(n-0.95)^{2}} & \text { Series II }
\end{array}
$$


-97-

Both series lead to nearly the same ionization energy of $11.41 \mathrm{eV}$. The small difference in limits possibly representing the splitting of the lowest ${ }^{2} \pi$ state of the ion. Using the same $\delta$ values, Dibeler et. al. have calculated the Rydberg series converging to each of the three vibrational states of $\mathrm{C}_{2} \mathrm{H}_{2}^{+}$. It is found that Series II correlates well with observed peaks. These series are also shown in Fig. 1. 


\section{REFERENCES}

I. V. H. Dibeler and R. M. Reese, J. Res. Nat. Bur. Stand., A68, 409 (1964).

2. A. J. C. Nicholson, J. Chem. Phys., 43, 1171 (1965).

3. R. Botter, V. H. Dibeler, J. A. Walker and H. M. Rosenstock, J. Chem. Phys., 44, 1271 (1966).

4. B. Brehm, 2. Naturforsch., 21a, 196 (:966).

5. I. Omura, T. Kaneko, Y. Yamada and K. Tarraka, J. Phys. Soc. Jap., 27, 178 (1969).

6. C. Biser and D. W. Turner, Pro. Roy. Soc. (London), A308, 19 (1968)

7. W. C. Price, Phys. Rev., 47, 444 (1935).

8. P. G. Wilkinson, J. Mol. Spectrosc., 2, 387 (1958).

9. F. P. Lossing, Int. J. Mass Spectrom. Ion Phys., 5, 190 (1970).

10. C. Y. Ng, B. H. Mahan and Y. T. Lee, J. Chem. Phys., (1976). 
-99-

Table I. Summary of Ionization Energies for Acetylene

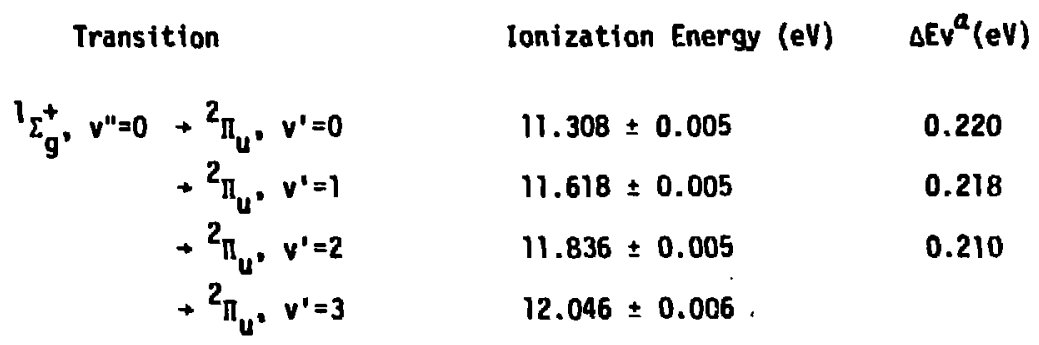

$a_{\triangle E V}$ is the vibrational spacing. 
$-100-$

FIGURE CAPTIONS

Fig. 1. The photofonization efficiency curve of acetylene $\left(\mathrm{C}_{2} \mathrm{H}_{2}\right.$ in the wavelength range from $1020 \AA$ to $1095 \AA(11.322 \mathrm{eV}$ $12.155 \mathrm{eV})$. 


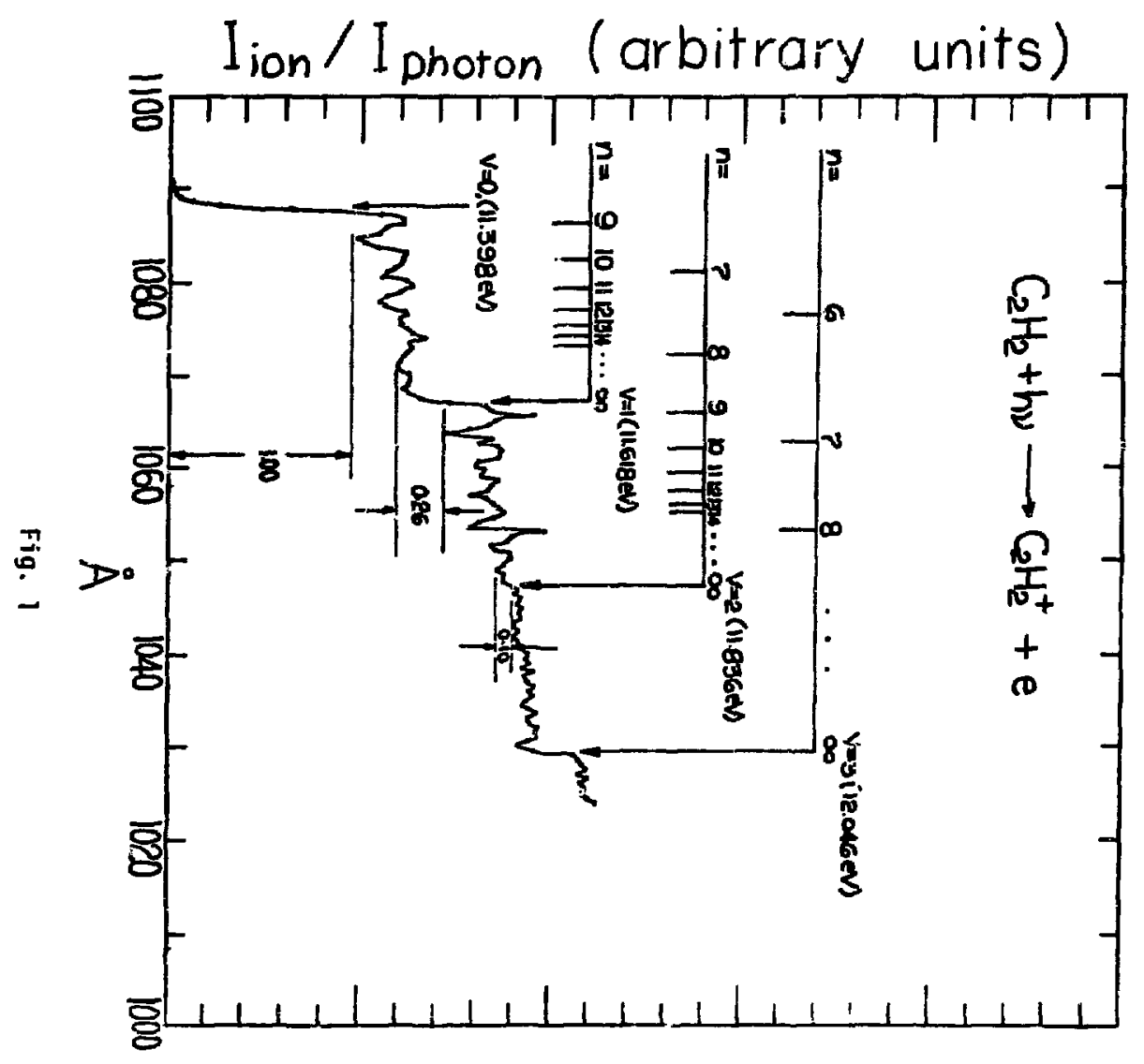


B. Photoionization Study of Methyl Iodide $\left(\mathrm{CH}_{3} \mathrm{I}\right)$

Autoionization is a familiar phenomenon in photoionization, but has been largely avoided in $21.22 \mathrm{eV}$ (HeI) photoelectron spectroscopy. The reason is that for ordinary gases there are relatively few strong autoionizing states at energies above $20 \mathrm{eV}$. Autoionization was found to be the major ionizing process for $\mathrm{CH}_{3} \mathrm{I}$ near the threshold from the previous photoionization studies. ${ }^{1,2}$ The iowest ionization potential of methyl iodide corresponds to the removal of an electron from a nonbonding (5p, e) orbital which is mainly localized on the iodine atom. This leaves the $\mathrm{CH}_{3} \mathrm{I}^{+}$molecular ion in the ${ }^{2} \mathrm{E}$ state which is further split by spin-orbit interaction into the ${ }^{2} E_{3 / 2}$ (ground) and ${ }^{2} \mathrm{E}_{1 / 2}$ states.

The experimental apparatus, arrangement and procedure was the same as previously described. ${ }^{3}$ Methyl iodide was introduced into the main chamber as a target gas by expansion through a $0.12 \mathrm{~mm}$ diameter pyrex nozzle at a stagnation pressure of 400 torr (the vapor pressure of methyl iodide at room temperature). The average counting rate was about 200 ct/sec. Counts were collected for $100 \mathrm{sec}$ at each point.

The photoionization efficiency curve for methyl iodide is shown in Fig. 1. The spectrum shows a large number of well defined autoionization peaks. It is found that this series is the same as obtained by Price ${ }^{4}$ from the analysis of the vacuum ultraviolet absorption spectruin of $\mathrm{CH}_{3} \mathrm{I}$. This series is fit by the Rydberg equation

$$
h v(e V)=10.157-\frac{13: 607_{4}}{(n-0.20)^{2}} \quad n=5-14
$$


The splitting in the two peaks corresponding to $n=5$ and 6 is suggested to be caused by the interaction of the Rydberg electron with the nonspherical charge distribution of the $\mathrm{CH}_{3} \mathrm{I}^{+}$core. ${ }^{2}$

The first ionization potential as determined from our experiment is $9.532 \pm 0.003 \mathrm{eV}$, which agrees favorably with the spectroscopic value. 4 The spin-orbit splitting of the ${ }^{2} \mathrm{E}$ molecular ionic state for $\mathrm{CH}_{3} \mathrm{I}^{+}$is expected to be mainly determined by the spin-orbit coupling coefficient of an $\mathrm{I}^{+}$atomic ion, since it is determined by the group $5 \mathrm{p}^{3}$ localized on $\mathrm{I}^{+}$. The spin-orbit coupling constant determined for $\mathrm{CH}_{3} \mathrm{I}^{+}$from this experiment is $0.625 \pm 0.003 \mathrm{eV}$ which is neariy the same as the splitting of simflar doublet in $\mathrm{ICl}^{+5}(0.630 \mathrm{eV})$. 


\section{REFERENCES}

1. J. P. Morrison, H. Hurzeler and M. G. Inghram, J. Chem. Phys., 33. 821 (1960).

2. T. Baer and B. P. Tsai, J. of Electron Spect. and Related Phenomena, 2, 25 (1973).

3. C. Y. Ng, B. H. Mahan and Y. T. Lee, J. Chem. Phys., (1976).

4. H. C. Price, J. Chem. Phys., 4, 536 (1936).

5. C. Y. Ng, B. H. Mahan and Y. T. Lee, J. Int. Mass Spect. and Ion Phys., to be published. 


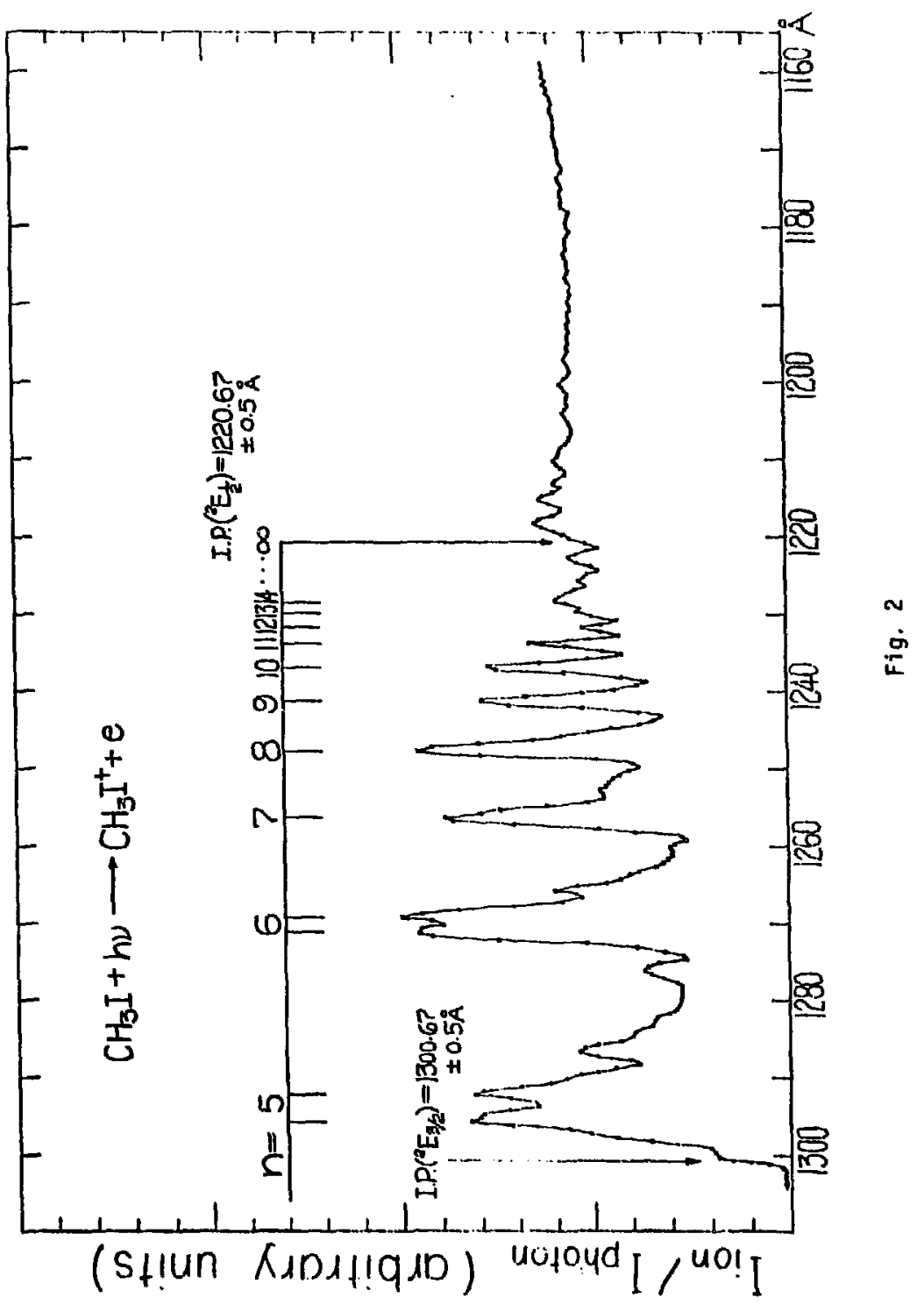


VIII. IMPROVED POTENTIALS FOR $\mathrm{Ne}+\mathrm{Ar}, \mathrm{Ne}+\mathrm{Kr}$ AND $\mathrm{Ne}+\mathrm{Xe}$

\section{ABSTRACT}

Improved interatomic potentials for Ne-rare gas pairs have been obtained by fitting a multiparameter potential to low energy differential cross sections, second virial coefficients and diffusion coefficients. All asymetric Ne-rare gas potentials have narrower attractive wells than those of the symmetric rare gas pairs. The values of $r_{m}$ and $E$ for $\mathrm{Ne}+\mathrm{Ar}, \mathrm{Ne}+\mathrm{Kr}$ and $\mathrm{Ne}+\mathrm{Xe}$ are $3.43 \AA, 719^{\circ} \mathrm{K} ; 3.58 \AA, 74.5^{\circ} \mathrm{K}$ and $3.75 \AA, 75.0^{\circ} \mathrm{K}$, respectively. 
INTRODUCTION

Potential parameters for $\mathrm{Ne}+\mathrm{Ar}$, $\mathrm{Ne}+\mathrm{Kr}$, and $\mathrm{Ne}+\mathrm{Xe}$ have been previously determined by fitting $L J(n, 6)$ potentials to the differential elastic scattering cross sections. 1 Parson et. al. ' have been able to resolve oscillations in their differential cross section measurenk'nts and to discriminate against the $\operatorname{LJ}(8,6)$ and the $\operatorname{LJ}(12,6)$ in favor of the $L J(20,6)$ potential for these systems. Al though the $\operatorname{LJ}(20,6)$ potential is not expected to provide an adequate description for the long range attraction and shor* range repulsion, the $L J(20,6)$ potentials with suitable values of $r_{m}$ and $\varepsilon$ do provide differential cross sections in goad agreemerit with experimental results for all $\mathrm{Ne}+\mathrm{Ar}$, Ne $+\mathrm{Kr}$, and He + Xe systems at thermal energies. Thus, it is concluded that the narrower $L J(20,6)$ potentials should provide a good description of the shape of the attractive well, and the parameters ( $r$ and $r_{m l}$ ) obtained must be quite realistic. The striking results that well deptlis of $\mathrm{Ne}+\mathrm{Ar}, \mathrm{Ne}+\mathrm{Kr}$, and $\mathrm{Ne}+\mathrm{Xe}$ are almost identical, as observed in that work, were somewhat unexpected. Since the commonly used geometric combining rule for well depths of asymmetric systems from corresponding symetric pairs predicts a significantly deeper well for Ne + Xe compared to Ne $+\mathrm{Ar}$, some questions have been raised regarding the reliability of these potential parameters and the sensitivity of experimental angular distributions of these systems to the potential parameters. ${ }^{2}$ We have made further investigations of these systems by carrying out differential cross section measurments at lower collifsion energies than in the previous work and have derived more precise 
multiparameter piecewise potentials in the form of MSV or ESMSV, 3 which provide good agreement with both experimental differential cross sections, second virial coefficients and diffusion coefficients.

\section{EXPERIMENTAL}

The experiments were carried out by crossing two supersonic beams of noble gas atoms, as described previously. ${ }^{3}$ The Ne beam was produced from a cryogenic beam source ${ }^{4}$ cooled with liquid nitrogen. The source pressure of the primary beam was about 450 torr at $77^{\circ} \mathrm{K}$ while that of the secondary beam was about 500 torr for $\mathrm{Ar}$ and $\mathrm{Kr}$, and 300 torr for $X e$ at room temperature. The angular distributions were obtained by counting elastically scattered Ne atoms for $40 \mathrm{sec}$ at each angle between $3^{\circ}$ and $40^{\circ}$. Each mixed pair was scanned three times, and the angular distributions obtained were averaged and are shown in Fig. 1. In order to correct for drifting and fluctuation of the beams and detector sensitivity, we returned to the reference angle $\left(10^{\circ}\right.$ from the Ne beam) frequently during the scan, and data were time normalized. The standard deviations of $40 \mathrm{sec}$ counts at the reference angle were less than 2\%. In the $\mathrm{Ne}+\mathrm{Kr}$ and $\mathrm{Ne}+\mathrm{Xe}$ experiments, $\mathrm{Ne}$ was detected at $\mathrm{M} / \mathrm{e}=20\left(\mathrm{Ne}^{+}\right)$, but in $\mathrm{Ne}+\mathrm{Ar}$ experiments $\mathrm{Ne}$ was detected at $\mathrm{M} / \mathrm{e}=$ $10\left(\mathrm{Ne}^{++}\right)$. It was found that the differential cross section for $\mathrm{Ne}+$ $A \dot{r}$, obtained by detecting $\mathrm{Ne}^{+}$, is significantly different from that obtained by detecting $\mathrm{Ne}^{++}$at wide angles when both $\mathrm{Ne}$ and $\mathrm{Ar}$ beams are at room temperature. With the liquid nitrogen cooled Ne beam, 
however: the differences are only detectable at ?aboratory angles larger than $25^{\circ}$ from the Ne beam. A simple kinematic analysis shows that this is due to be simultaneous detection of elastically scattered $\mathrm{Ne}$ as $\mathrm{Ne}^{+}$and $\mathrm{Ar}$ as $\mathrm{Ar}^{++}$at $\mathrm{M} / \mathrm{e}=20$. Detection of $\mathrm{Ne}$ as $\mathrm{Ne}^{++}$is free from contamination of $\mathrm{Ar}$, since $\mathrm{Ar}^{+4}$ is not produced in our detector using a $200 \mathrm{eV}$ electron beam.

RESULTS AND ANALYSIS

The experimental results of $\mathrm{Ne}+\mathrm{Kr}$ and $\mathrm{Ne}+\mathrm{Xe}$ were fitted by varying eight parameters $\left(\varepsilon, r_{m}, \beta, c_{6}, c_{8}, x_{3}, x_{4}\right)$ of the piecewise Morse-Spline-van der Waals potentials (MSV), as mentioned before. ${ }^{3}$ For $\mathrm{Ne}+\mathrm{Ar}$, since information on the repulsive part of the potential is available, the exponential form is used in the high energy region (1.1 to $10 \mathrm{eV})$, and connected smoothly by an exponential Spline function to a Morse function. The reduced form of the exponential-Spline-MorseSpline-van der Waals (ESMSV) potential is written as follows:

$$
\begin{aligned}
& f(x)=V(r) / \varepsilon \quad x=r / r_{m} \quad C_{n}=C_{n} / \varepsilon r_{m}^{n} \\
& f=\operatorname{Aexp}[-a(x-1)] \\
& 0 \therefore x=x_{1} \\
& f=\exp \left(a_{1}+\left(x-x_{1}\right)\left\{a_{2}+\left(x-x_{2}\right)\left[a_{3}+\left(x-x_{1}\right) a_{4}\right]\right\}\right) \\
& \text { (Exponential Spline function) } x_{1} \cdots x \cdots x_{2} \\
& f=\exp [-2 \beta(x-1)]-2 \exp [-\beta(x-1)] \\
& x_{2} \cdot x \cdot x_{3}
\end{aligned}
$$




$$
\begin{array}{rlr}
f=b_{1}+\left(x-x_{3}\right)\left\{b_{2}+\right. & \begin{aligned}
\left.\left(x-x_{4}\right)\left[b_{3}+\left(x-x_{3}\right) b_{4}\right]\right\} \\
\text { (Cubic Spline function) }
\end{aligned} & x_{3}<x<x_{4} \\
f=-c_{6} x^{-6}-c_{8} x^{-8}-c_{10} x^{-10} & x_{4} \leq x<\infty
\end{array}
$$

Differential cross sections were calculated using the JWKB method of approximating phase shifts. A nonlinear least squares fitting technique by Marquardt ${ }^{5}$ was used to obtain initial estimates of $\varepsilon, r_{m}$, $B$ for fixed $x_{3}$ and $x_{4}$. Values of $x_{3}$ and $x_{4}$ are varied to change the shape of the outer wall of the well. $C_{6}$ constants are taken from the semiempirical calculation by Gordon et. al. ${ }^{6-8} C_{B}$ constants are the geometric means of the symmetric pairs, and $C_{10}$ constants are neglected because of lack of information. Thus, only five parameters are varied to fit the low temperature elastic differential cross sections. The value of $r_{m}$ can be well determined for a given potential function by matching the fast oscillations resolved in experimental angular distributions and those of theoretical calculations. Since low energy differential cross sections are quite sensitive to the shape of the attractive parts of the potentials, the locations of joining points, $x_{3}$ and $x_{4}$, must be chosen very carefully in order to give a good fit to the small angle differential cross sections. Finally $B$ and $\varepsilon$ are varied to obtain optimum values. This sequence of variation of parameters is repeated untf the calculated dfferential cross sections give excellent agreement with experimental results. In the process of finding the best parameters, second virial coefficients were also calculated and compared with experimental data.9,10 Although for Ne + 
$\mathrm{Kr}$ and $\mathrm{Ne}+\mathrm{Xe}$ only a few second virial coefficient data points are available, these second virial coefficient data measured at relatively high temperatures are sensitive to the repulsive parts of the potentials and supplement low energy differential cross section data which are mainly sensitive to the attractive well. The ESMSV potential for $\mathrm{Ne}+\mathrm{Ar}$ and MSV potentials for $\mathrm{Ne}+\mathrm{Kr}$ and $\mathrm{Ne}+\mathrm{Xe}$, which give a good agreement with both the differential cross sections and the second virial coefficients are then used to calculate diffusion coefficients at various temperatures. The calculated diffusion coefficients for $\mathrm{Ne}+\mathrm{Kr}$ and $\mathrm{Ne}+\mathrm{Xe}$ give excel lent agreement with the experimental measurements of Hogervorst, ${ }^{28}$ but for $\mathrm{Ne}+\mathrm{Ar}$, al though the agreement is excellent up to $700^{\circ} \mathrm{K}$, the calculated diffusion coefficients are $4.1 \%$ higher than the experimental value at $1000^{\circ} \mathrm{K}$ and the deviation increases to $5.9 \%$ at $1400^{\circ} \mathrm{K}$. The small but significant discrepancies at very high temperatures indicate that the high energy repulsive part of the potential of $\mathrm{Ne}+$ Ar could further be improved, Consequently, three parameters, $A, \alpha$ and $x_{1}$, related to the exponential repulsion are adjusted until the calculated diffusion coefficients also agree with the experimental values. The best fit ESMSV potential for $\mathrm{Ne}+$ $\mathrm{Ar}$ and MSV potentials for $\mathrm{Ne}+\mathrm{Kr}$ and $\mathrm{Ne}+\mathrm{Xe}$ are shown in Fig. 2 . The parameters of these potentials are listed in Table I. The differential cross sections calculated from the best potentiais obtained are also compared with experimental results in Fig. 1. The second virial coefficients were calculated by Hirschfelder, Curtiss, and Bird, ${ }^{12}$ including two quantum corrections. For Ne-rare gas 
systems, quantum corrections are relatively small compared to classical calculations in the temperature range in which comparisons were made. The standard deviations of the best parametric potentials for $\mathrm{Ne}+\mathrm{Ar}$, $\mathrm{Ne}+\mathrm{Kr}$, and $\mathrm{Ne}+\mathrm{Xe}$ fitted to the second virial coeffictents are $0.81,0.439$, and $0.648 \mathrm{cc} / \mathrm{mole}$, respectively, and these values are considered to be satisfactory in view of the uncertainty of the second virial coefficient data. The calculated second virial coefficients are compared with the experimental results in Fig. 3 as a function of temperature.

\section{DISCUSSION}

The reduced masses of $\mathrm{Ne}+\mathrm{Ar}$, $\mathrm{He}+\mathrm{Kr}$, and $\mathrm{Ne}+\mathrm{Xe}$ are relatively small, and at a reduced collision energy $(E / E)$ around five, pronounced rainbow structures are only observed in the $\mathrm{Ne}+\mathrm{Xe}$ system. In finding a potential from a differential cross section, it is very imporcant to recognize that the relative intensities at all angles, as well as positions of extrema in differential cross sections, must be matched precisely. If the potential functions assumed are not realistic, one often finds that by varying the parameters it is possible to match the locations of extrem but not the relative intensity at all angles. Amplitudes of extrema, such as fast oscillations, rainbow oscillations, and symmetry oscillations, are usually damped by finite velocity spreads In the beam and finite angular resolution of the experimental arrangenent. Consequently, in order to make a proper comparison, differential cross 
sections calculated from a given potential should be averaged over experimental conditions. Values of $\varepsilon$ and $r_{m}$ determined for $\mathrm{Ne}+\mathrm{Ar}$, $\mathrm{Ne}+\mathrm{Kr}$, and $\mathrm{Ne}+\mathrm{Xe}$ in this work, are estimated to be better than $3 \%$. In Fig. 4, sensitivity of differential cross sections for $\mathrm{Ne}+\mathrm{Ar}$ to the values of $\varepsilon$ and $r_{m}$ are illustrated. Fig. 4a shows a series of calculations changing only $r_{m}$ by $5 \%$ in both directions from the best MSV potential determined. The difference in the frequency and position of fast oscillations is quite noticeable. In Fig. $4 \mathrm{~b}, E$ is changed $7 \%$ from the best value, and appreciable changes in the slope of differential cross sections are observable. By keeping the product of $\varepsilon$ and $r_{m}$ nearly constant and changing individual parameters 5 to $7 \%$ the general fall-off in angular distributions is found to be quite similar, as shown in Fig. 4c. Nevertheless, the difference in oscillation frequency and amplitude can be detected. Calculations shown in Fig. 4 are all averaged over experimental conditions.

It is interesting to note that $\varepsilon$ and $r_{m}$ values determined in this work are within the uncertainty of previous work using a $L J(20,6)$ potential form. Recently. in order to make a comparison with the experimental results of Parson et. al., Brown and Munn ${ }^{2}$ calculated differentlal cross sections for the mixed inert gas systems $\mathrm{Ne}+\mathrm{Ar}$. $\mathrm{Ne}+\mathrm{Kr}$, Me $+\mathrm{Xe}, \mathrm{Ar}+\mathrm{Kr}$, and $\mathrm{Ar}+\mathrm{Xe}$, using a potential $\mathrm{V}(r)=$ $\varepsilon \phi(r / \sigma)$ proposed by Munn ${ }^{13-15}$ and a set of combining rules proposed by Kohler $^{16}$ for the specific energy and length scaling parameters:

$$
\begin{aligned}
& \sigma_{i j}=\left(\sigma_{i j}+\sigma_{j j}\right) / 2 \\
& \varepsilon_{i j}=2 a_{j} a_{j} / \sigma_{i j}{ }^{6}\left(\gamma_{i j}+\gamma_{j j}\right)
\end{aligned}
$$


where

$$
\gamma_{i j}=\left(\alpha_{i}^{2} / \varepsilon_{i j} \sigma_{i i}^{6}\right) \text {, }
$$

$\varepsilon$ and $\sigma$ are the depth of the potential minimum and position of the potential zero, and $\alpha$ is the polarizability.

They stated that the gross rainbow structure of the scattering cross sections was reproduced by their calculations and that there was substantial agreement with the high frequency oscillations observed in the neon series. They concluded that the lack of good agreement between their mixed interaction potential parameters (derived from virial and transport data and the use of the combination rule) and those derived by Parson et. ai. Indicates that more theoretical and experimental work is needed to unequivocally determine the mixed and pure rare gas interactions.

Actually, the disagreement between the differential cross sections calculated with their potentials and those measured by Parson et. al. is quite appreciable, and the differences in differential cross sections do reflect the differences in the proposed well depths. In the work of Brown and Munn, the experimental results are compared with a calculation for a single relative velocity. Thus, the differences between the calculation and the data are somewhat obscured by the large amplitude and high frequency oscillations, which are severely damped in the experiment.

The values of $\varepsilon$ and $\sigma$ (defined as $V(\sigma)=0$ ) are compared with commonly used combination rules and listed in Table II. The simple combination rules do not give good estimates for these systems. As 
in He-rare gas systems, ${ }^{17}$ the Ne-rare gas systems have nearly the same well depths, and the reciprocal relation for $E_{;} \varepsilon_{i j}=\varepsilon_{i j} \varepsilon_{j j} /\left(\varepsilon_{i j}+E_{j j}\right)$ gives somewhat better predictions that other combination rules but cannot be regarded as adequate. The reduced forms of all Ne-rare gas systems are plotted in Fig. 5 and are also tabulated in Table III. The differences between the $\mathrm{Ne}_{2}$ potential and asymmetric Ne-rare gas systems are as significant as the differences among asymmetric Ne-rare gas systems. The Ne-Xe potential is more attractive at large distances and more repulsive at short distances than the Ne-Ar potential.

Some previously proposed potentials ${ }^{18-26,28}$ for $\mathrm{Ne}-\mathrm{Ar}, \mathrm{Ne}-\mathrm{Kr}$, and Ne-Xe are tabulated in Tables IV, V, and VI for comparison. These potentials are mainly constructed from transport properties and semiempirical calculations. Some Morse- 6 and exp-6 potentials are in fair agreement-with MSV potentials dervied in this work. Differential cross sections calculated for some of these potentials are shown in Figs. 6 and 7 ; the potentials themselves are shown in Figs. 8 and 9.

For Ne + Ar the Morse-6 potential of Gaydaenko et. al. ${ }^{25}$ (No. 3 in Table IV) was shown by konowalow et. al. ${ }^{19}$ to give a rather good fit to the second virial coefficients; the differential cross section calculated from this potential is also quite close to experimental results except that the general fall-off of the differential cross section as a function of the scattering angle seems somewhat faster. Diffusion coefficients in the temperature range in which the average relative kinetic energy of atoms is much higher than the potential well depth are mare sensitive to the repulsive part of the potential than the 
attractive part, since differential cross sections are weighted by a $(1-\cos \theta)$ factor in the total diffusion cross section, thus reducing the importance of the contribution from small angle scattering, which is more sensitive to the attractive part of the potential. Various exp-6 potentials, obtained from idffusion coefficients by R. J. J. van Heijningen et. al. ${ }^{18}$ over the temperature range from $100^{\circ} \mathrm{K}$ to $400^{\circ} \mathrm{K}$, do agree more closely in the repulsive part of the potential than the potential well. As shown in Fig. 8(b), the ESMSV potential has substantial overlap with these potentials in the repulsive region, especially when $\alpha=15$. For the Ne-Kr potential, an exp-6 potential with $\alpha=15^{18}$ agrees almost exactly in the repulsive region with the MSV potential derived in this work as shown in Fig. 9. Potential parameters for $L J(12,6)$ and exp-6 potentials have been determined by Hogervorst ${ }^{28}$ from accurate measurements of diffusion coefficients between 100 and $1600^{\circ} \mathrm{K}$. The well depth, $\varepsilon$, for Ne-Ar obtained in his work is about 10\% smaller than our present work, but both $E$ and $r_{m}$ for $\mathrm{Ne}-\mathrm{Kr}$ and $\mathrm{Ne}-\mathrm{Xe}$ agree with our present work within the uncertainties of the determinations of these parameters. The facts that nearly identical values of $\varepsilon$ and $r_{m}$ are obtained for both $L J(12,6)$ and exp-6 potential forms in Hogervorst's work led him to conclude that the diffusion coefficients could provide an estinate of $\varepsilon$ and $r_{m}$ together with the steepness of the repulsive part of the potentials but not the detalled information on the form of the potentials. The form of the potential, especially around the attractive well, can be better determined from the measurements of elastic differential cross sections. 
ACKNOWLEDGEMENTS

This research was supported by the U. S. Atomic Energy Commision and in part by the Advanced Research Projects Agency, Louis Block Fund of The University of Chicago. YTL acknowledges the Teacher-Scholar Grant from the Camille and Henry Dreyfus Foundation. 


\section{REFERENCES}

1. J. M. Parson, T. P. Schafer, F. P. Tully, P. E. Siska, Y. C. Wong and Y. T. Lee, J. Chem. Phys., 53, 2123 (1970). R. W. Bickes, Jr. and R. B. Bernstein, Chem. Phys. Letters, 4, 111 (1970).

2. N. J. Brown and R. J. Munn, J. Chem. Phys., 57, 2216 (1972).

3. J. M. Parson, P. E. Siska and Y. T. Lee, J. Chem. Phys., 56, 1511 (1972).

4. J. M: Farrar and Y. T., Lee, J. Chem. Phys., 56, 5801 (1972).

5. D. W. Marquardt, J. Soc. Ind. App. Math., 11, 431 (1963).

6. R. G. Gordon, J. Chem. Phys., 48,3929 (1968).

7. G. Starkschall and R. G. Gordon, J. Chem. Phys., 54, 663 (1971).

8. P. W. Langhoff, R. G. Gordon and M. Karplus, J. Chem. Phys., 55, 2126 (1971).

9. J. Brewer and R. G. Vaughn, J. Chem. Phys., 50, 2960 (1969).

10. J. Brewer, Air Force Office of Scientific Research Report No. 67-2795 (1967).

11. F. T. Smith, Phys. Rev., A5, 1708 (1972).

12. J. O. Hirschfelder, C. F. Curtiss and R. B. Bird, Molecular Theory of Gases and Liquid (Wiley, New York 1964), Chapter 6.

13. R. J. Munn, J. Chem. Phys., 40, 1439 (1964).

14. R. J. Munn and F. J. Smith, J. Chem. Phys., 43, 3998 (1965).

15. M. L. Klein and R. J. Munn, J. Chem. Phys., 47, 1035 (1967).

16. F. Kohler, Monatsh. Chem., 88, 857 (1957).

17. C. H. Chen, P. E. Siska and Y. T. Lee, J. Chem. Phys., 59, 601 (1973). 
18. R. J. J. van Heijningen, J. P. Harpe and J. J. M. Beenakker, Physica, 38, 1 (1968).

19. D. D. Konowalow and D. S. Zakheim, J. Chem. Phys., 57, 4375 (1972).

20. D. D. Konowalow, J. Chem. Phys., 50, 12 (1969).

21. B. N. Srivastava and K. P. Srivastava, J. Chem. Phys., 30, 984 (1969).

22. R. K. Nesbet, J. Chem. Phys., 48, 1419 (1968).

23. E. A. Mason, J. Chem. Phys., 23, 49 (1955).

24. K. P. Srivastava, J. Chem. Phys., 28, 543 (1958).

25. V. I. Gaydaenko and V. K. Nikulin, Chem. Phys. Letter, I., 360 (1970).

26. R. L. Matcha and R. K. Nesbet, Phys. Rev., 160, 72 (1967).

27. J. M. Farrar, Y. T. Lee, V. V. Goldman and M. L. Klein, Chem. Phys. Letters, 19, 359 (1973).

28. W. Hogervorst, Physica, 51, 59 (1971). 


\section{FIGURE CAPTIONS}

Fig. 1. Laboratory angular distributions of elastically scattered Ne for the $\mathrm{Ne}+\mathrm{Ar}$, Ne $+\mathrm{Kr}$ and $\mathrm{Ne}+\mathrm{Xe}$ systens. Solid curves are calculated from best fit potentials.

Fig. 2. Potentials of $\mathrm{Ne}+\mathrm{Ar}, \mathrm{Ne}+\mathrm{Kr}$ and $\mathrm{Me}+\mathrm{Xe}$ obtained in this work.

Fin. 3. Comparison of second virial coefficients calculated fron best fit potentials with experimental data.

Fig. 4. Sensitivity of differential cross sections of Ne + Ar to the variation of $\varepsilon$ and $r_{m}$ for ESirs potentials listed in Table I.

(a) Varlation of $r_{m}$, keeping $\varepsilon$ constant.

(b) Variation of $\varepsilon$, keeping $r_{m}$ constant.

(c) Simultaneous variation of $r_{m}$ and $\varepsilon$, keeping $\varepsilon r_{m}$ nearly constant.

Fig. 5. Reduced potentials of Ne + He, Ne-Ar, Ne $+\mathrm{Kr}$ and $\mathrm{Me}+\mathrm{Xe}$.

Fig. 6. Laboratory angular distributions of elastically scattered Ne for the $\mathrm{Ne}+$ Ar system compared with previousiy proposed potentials. Mumbered curves are calculated from corresponding potentials of Table IV. The experimental data are shown twice to allow a clear comparison with the calculated results.

Fig. 7. Laboratory angular distributions of elastically scattered Ne for the Me + Kr system is compared with a previously proposed Ne + Kr potential Ifsted in Table V. (No. II). 
$.121-$

Fig. 8. Comparison of fitted ESHSV potential (30) id curves) with previously proposed potentials for Ne + Ar. The fitted curve is replotted twice for ease of comparison. Numbering scheme corresponds to that of Table IV.

fig. 9. Comparison of fitted MSV potential with o previously proposed Me $+\mathrm{kr}$ potential listed in Table $\mathrm{V}$ (Ho. II). 
TABLE I.

ESMSV POTENTIAL PARAMETERS

\begin{tabular}{|c|c|c|c|c|}
\hline & $\mathrm{Ne}-\mathrm{Ne}$ & $\mathrm{Ne}-\mathrm{Ar}$ & $\mathrm{Ne}-\mathrm{Kr}$ & $\mathrm{Ne}-\mathrm{Xe}_{\mathrm{e}}$ \\
\hline e (Kcal/molo $)$ & .085 & 0.143 & 0.148 & 0.149 \\
\hline$r_{m}(X)$ & 3.11 & 3.43 & 3.58 & 3.745 \\
\hline$\beta$ & 2.032 & 6.6 & 7.0 & 7.9 \\
\hline $\mathbf{A}$ & .132 & 0.245 & -- & $-\infty$ \\
\hline - & 4.0 & 17.55 & $-\infty$ & $-\infty$ \\
\hline$C_{6}$ (Keal/mole $\left.A^{6}\right)$ & 90.4 & 282 & 392.5 & 570 \\
\hline$\left.C_{g}(\text { Kcal/mole })^{8}\right)$ & 220.6 & 590 & 810 & 1210 \\
\hline $\mathrm{C}_{10}\left(\mathrm{Kcol}_{\mathrm{mole}} \mathrm{I}^{10}\right)$ & -ne- & $-\infty$ & $-\cdots$ & $-\infty$ \\
\hline$n_{1}$ & 6.66 & 2. 106 & $-\infty$ & -- \\
\hline$\sigma_{2}$ & -17.05 & -36.07 & $-\infty$ & $-\infty$ \\
\hline$G_{3}$ & -36.32 & -251.69 & $-\infty$ & $-\infty$ \\
\hline$G_{4}$ & -14.119 & -747.52 & $m$ & --- \\
\hline b) & -7.5 & -0.61 & -.865 & -.92 \\
\hline$b_{2}$ & 1.976 & 1.89 & 1.259 & 1.083 \\
\hline$b_{3}$ & -3.999 & -5.59 & -2.997 & -2.462 \\
\hline$b_{4}$ & -.1522 & 5.64 & 2.074 & 1.555 \\
\hline$x_{1}$ & 0.5 & 0.8 & $-\infty$ & $\ldots$ \\
\hline$x_{2}$ & 0.826 & 0.88 & --- & - \\
\hline$x_{3}^{2}$ & 1.1097 & 1.148 & 1.065 & 1.042 \\
\hline$x_{4}$ & 1.4 & 1.36 & 1.71 & 1.86 \\
\hline
\end{tabular}


TABLE II

Fatential Paremeters from Combination Rules Compared with Experiment.

\begin{tabular}{|c|c|c|c|c|c|c|c|c|c|c|c|}
\hline \multirow[b]{2}{*}{ System $^{(a)}$} & \multicolumn{4}{|c|}{ Internucleor Distance (b) } & \multirow[b]{2}{*}{$e_{i i}$} & \multirow[b]{2}{*}{$e_{i l}$} & \multirow[b]{2}{*}{$e_{i}^{(c)}$} & \multirow[b]{2}{*}{$\varepsilon_{i j}^{(d)}$} & \multirow[b]{2}{*}{$\varepsilon_{i j}^{(e)}$} & \multirow[b]{2}{*}{$e_{i j}^{(i)}$} & \multirow[b]{2}{*}{$\varepsilon_{i j}($ Expt $)$} \\
\hline & $\sigma_{i i}$ & $\sigma_{\mathrm{iI}}$ & $\sigma_{i j}^{(b)}$ & $\sigma_{i j}{ }^{(\text {Expt })}$ & & & & & & & \\
\hline Ne-Ar & $2.75^{(h)}$ & $3.34^{(9)}$ & 3.05 & 3.09 & 42.7 & 143 & 73.6 & 78.14 & 75.18 & 65.75 & 71.94 \\
\hline$N_{e-K r}$ & 2.75 & $3.64^{(h)}$ & 3.20 & 3.22 & 42.7 & 198 & 83.92 & 91.94 & 85.92 & 70.26 & 74.47 \\
\hline Ne-Xe & 2. 75 & $3.81^{(h)}$ & 3. $2 B$ & 3.42 & 42.7 & 276 & 93.06 & 108.5 & 100.2 & 73.94 & 74.98 \\
\hline
\end{tabular}

(a) The subseripts $i \equiv N e, i \equiv$ rare gas portner

(b) $\sigma_{\mathrm{ii}}=\frac{1}{2}\left(\sigma_{\mathrm{ii}}+\sigma_{\mathrm{if}}\right)$

(c) $\varepsilon_{i j}=2 a_{i} a_{i} / \sigma_{i j}{ }^{6}\left(r_{i j}+r_{i j}\right) ; a_{i}, a_{i}, a_{i}$ are atomic polarizability $i \gamma_{i j}=\left(a_{i}{ }^{2} / e_{i j}=\sigma_{i j}{ }^{b}\right)_{i} z_{i j}$ abtoined (from (b).

(d) $e_{i j}=\left(e_{i i} \xi_{i j}\right)^{1 / 2}$.

(e) $e_{i j}=\left(\frac{1}{\sigma_{i j}}\right)^{6}\left[\varepsilon_{i i} \sigma_{i i}{ }^{6} \varepsilon_{i i} \sigma_{i i}{ }^{6}\right]^{T / 2} ; \sigma_{i j}$ abtoined from (o).

(f) $z_{i j}=2 \varepsilon_{i i} \xi_{i j} /\left(e_{i i}-z_{i f}\right)$.

(G) Ref. 27

(h) Ref. 28 
TAqLe III.

ESMSV REDUCED POTENTIAL

\begin{tabular}{|c|c|c|c|c|}
\hline$x$ & No-Ne & Ne-At & $N_{0}-K_{r}$ & No-Xo \\
\hline 0,50 & $7.807 \times 10^{2}$ & $1.590 \times 10^{3}$ & $1.090 \times 10^{3}$ & $2.593 \times 10^{3}$ \\
\hline 0.55 & $4.147 \times 10^{2}$ & $6.611 \times 10^{2}$ & $4.979 \times 10^{2}$ & $1.154 \times 10^{3}$ \\
\hline 0.60 & $2.12 \times 10^{2}$ & $2.749 \times 10^{2}$ & $2.375 \times 10^{2}$ & $5.084 \times 10^{2}$ \\
\hline 0.65 & $1.014 \times 10^{2}$ & 1. $143 \times 10^{2}$ & $1.111 \times 10^{2}$ & $2.20 \times 10^{2}$ \\
\hline 0.70 & $4.423 \times 10^{1}$ & $4.753 \times 10^{1}$ & $5.035 \times 10^{1}$ & $9.304 \times 10^{1}$ \\
\hline 0.75 & $1.710 \times 10^{1}$ & $1.976 \times 10^{1}$ & $2.161 \times 10^{1}$ & $3.752 \times 10^{\prime}$ \\
\hline 0.80 & 5.702 & 8.218 & 8. 336 & $1.386 \times 10^{1}$ \\
\hline 0.85 & 1.498 & 3.460 & 2.451 & $4.156 \times 10$ \\
\hline 0.90 & $-2.233 \times 10^{-1}$ & $-1.262 \times 10^{-1}$ & $2.77 \times 10^{-2}$ & $4,482 \times 10^{-1}$ \\
\hline 0.95 & $-8.619 \times 10^{-1}$ & $-8.47 \times 10^{-1}$ & $-6.244 \times 10^{-1}$ & $-7.654 \times 10^{-1}$ \\
\hline 1.00 & -1.000 & -1.000 & -1.000 & -1.000 \\
\hline 1.05 & $-9.266 \times 10^{-1}$ & $-9.210 \times 10^{-1}$ & $-9.128 \times 10^{-1}$ & $-8.957 \times 10^{-1}$ \\
\hline 1.10 & $-7.806 \times 10^{-1}$ & $-7.666 \times 10^{-1}$ & $-7.598 \times 10^{-1}$ & $-7.529 \times 10^{-1}$ \\
\hline 1.15 & $-6.3 \times 10^{-1}$ & $-6.058 \times 10^{-1}$ & $-6.25 \times 10^{-1}$ & $-6.27 \times 10^{-1}$ \\
\hline 1.20 & $-4.900 \times 10^{-1}$ & $-4.687 \div 10^{-1}$ & $-5.09 \times 10^{-1}$ & $-5.180 \times 10^{-1}$ \\
\hline 1.25 & $-3.73 \times 10^{-1}$ & $-3.623 \times 10^{-1}$ & $-4.1 \times 10^{-1}$ & $-4.24 \times 10^{-1}$ \\
\hline 1.30 & $-2.972 \times 10^{-1}$ & $-2.802 \times 10^{-1}$ & $-3.281 \times 10^{-1}$ & $-3.431 \times 10^{-1}$ \\
\hline 1.35 & $-2.270 \times 10^{-1}$ & $-2.2005 \times 10^{-1}$ & $-2.6 \times 10^{-1}$ & $-2.755 \times 10^{-1}$ \\
\hline 1.40 & $-1.762 \times 10^{-1}$ & $-1.754 \times 10^{-1}$ & $-2.048 \times 10^{-1}$ & $-2.188 \times 10^{-1}$ \\
\hline 1.45 & $-1.418 \times 10^{-1}$ & $-1.415 \times 10^{-1}$ & $-1.61 \times 10^{-1}$ & $-1.78 \times 10^{-1}$ \\
\hline 1.50 & $1.148 \times 10^{-1}$ & $-1.147 \times 10^{-1}$ & $-1.264 \times 10^{-1}$ & $-1.358 \times 10^{-1}$ \\
\hline
\end{tabular}


IABI.E III. --Continued.

$\mathbf{x}$

Ne-Ne

Ne-Ar

Ne-Kr

Ne-Xo

1. 60

$-7.697 \times 10^{-2}$

$-7.720 \times 10^{-2}$

$-8.069 \times 10^{-2}$

$-8.170 \times 10^{-2}$

1. 20

$-5.295 \times 10^{-?}$

$-5.326 \times 10^{-2}$

$-5.510 \times 10^{-2}-5.624 \times 10^{-2}$

1.80

$-3.725 \times 10^{-2}$

$-3.156 \times 10^{-2}$

$-5.808 \times 10^{-2}$

$-4.107 \times 10^{-2}$

1.90

$-2.673 \times 10^{-2}$

-2. $701 \times 10^{-2}$

$-2.778 \times 10^{-2}$

3. $030 \times 10^{-2}$

2. 00

$-1953 \times 10^{-2}$

$-1.976 \times 10^{-2}$

$-2.048 \times 10^{-2}$

$2.222 \times 10^{-2}$

2. 10

$-1.449 \times 10^{-2}$

$-1.469 \times 10^{-2}$

$-1.523 \times 10^{-2}-1.654 \times 10^{-2}$

2.20

$-1.091 \times 10^{-2}$

$-1.107 \times 10^{-2}$

$-1.148 \times 10^{-2}-1.249 \times 10^{-2}$

2. 30

$-8.319 \times 10^{-3}$

$-8.456 \times 10^{-3}$

$-8.770 \times 10^{-3}-9.547 \times 10^{-3}$

2. 40

$-6.421 \times 10^{-3}$

$-6.533 \times 10^{-3}$

$-6.777 \times 10^{-3}-7.384 \times 10^{-3}$

2. 50

$-5.009 \times 10^{-3}$

$-5.102 \times 10^{-3}$

$-5.294 \times 10^{-3}-5.772 \times 10^{-3}$

$\epsilon(\mathrm{Kcal} / \mathrm{mole}) \quad .085$

0. 143

0. 148

0. 149

$r_{m}(A)$

3. 11

3. 43

3. 58

3. 745 


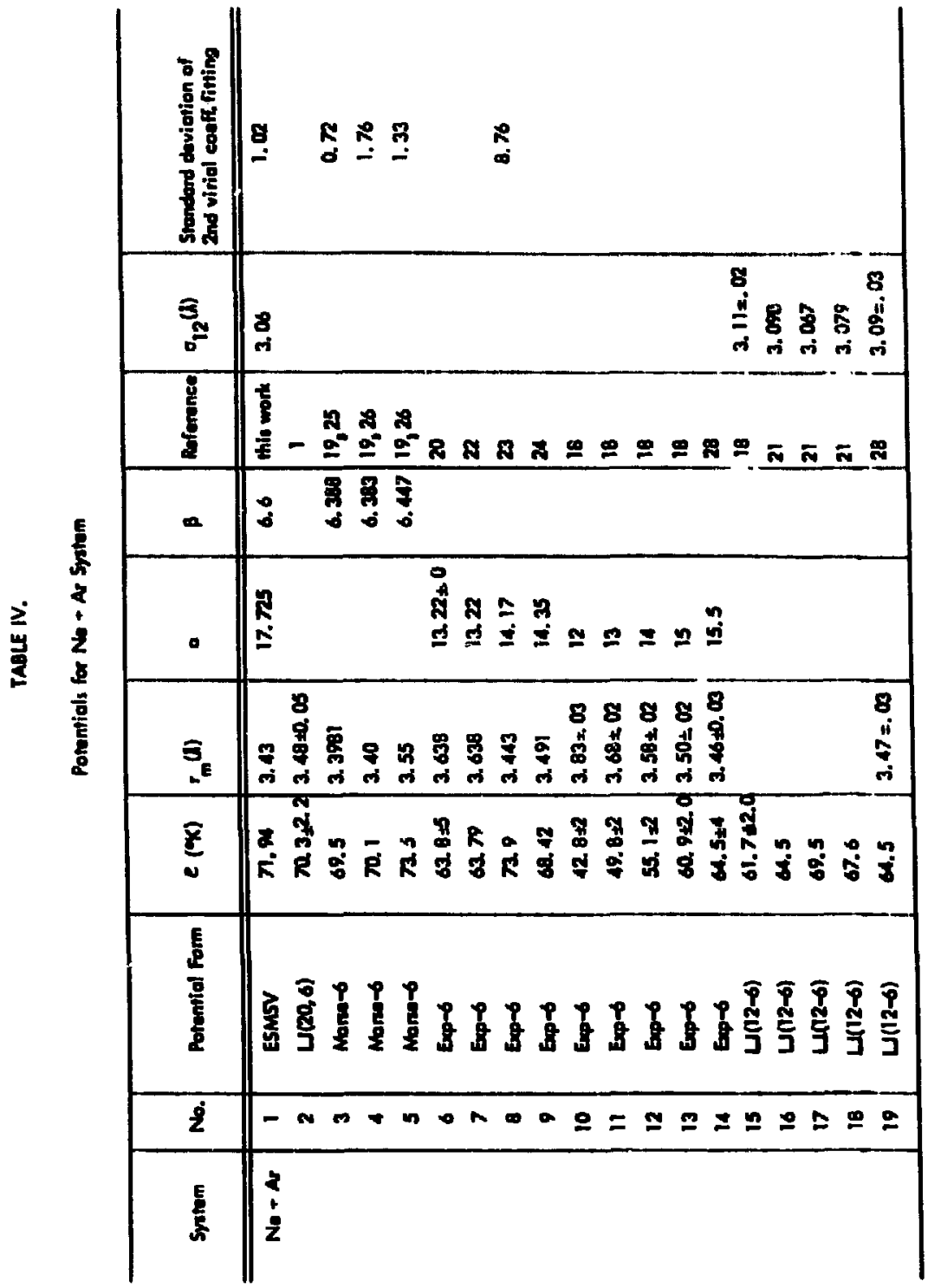


TABLE V.

Potentials for $\mathrm{No}_{0}+\mathrm{Kr}$ System

\begin{tabular}{|c|c|c|c|c|c|c|c|c|c|}
\hline System & No. & Polential Form & $e\left({ }^{\circ} K\right)$ & $r_{m}(f)$ & 0 & $\beta$ & $={ }_{12}(h)$ & Reference & $\begin{array}{l}\text { Stondord doviation s: } \\
\text { 2nd viriol couff firting }\end{array}$ \\
\hline $\mathrm{Ne}+\mathrm{K}_{\mathbf{r}}$ & $\begin{array}{c}1 \\
2 \\
3 \\
4 \\
5 \\
6 \\
7 \\
8 \\
9 \\
10 \\
11 \\
12\end{array}$ & $\begin{array}{l}M S V \\
U(20,6) \\
U(12,6) \\
U(12,6\} \\
U(12,6) \\
U(12,6) \\
L J(12,6) \\
\text { Exp-6 } \\
\text { Exp-6 } \\
\text { Exp-6 } \\
\text { Exp-6 } \\
\text { Exp-6 }\end{array}$ & $\begin{array}{l}74.47 \\
78.222 .2 \\
67.5 \\
63.5 \\
64.9 \\
69.8 \pm 3.5 \\
71.5 \pm 3.5 \\
52.8 \pm 3.5 \\
60.5 \pm 3.5 \\
65.8 \pm 3.5 \\
72.0 \pm 3.5 \\
71.5 \pm 4\end{array}$ & $\begin{array}{l}3.58 \\
3.6=.07 \\
\\
3.62=03 \\
3.94 \pm 04 \\
3.80 \pm .03 \\
3.71 \pm 03 \\
3.62 \pm .03 \\
3.60 \pm .03\end{array}$ & $\begin{array}{l}12 \\
13 \\
14 \\
15 \\
15.5\end{array}$ & 7.0 & $\begin{array}{l}3.226 \\
3.257 \\
3.291 \\
3.287 \\
3.24=02 \\
3.22=.03\end{array}$ & $\begin{array}{c}\text { this work } \\
1 \\
21 \\
21 \\
21 \\
18 \\
28 \\
18 \\
18 \\
18 \\
18 \\
28\end{array}$ & 3.00 \\
\hline
\end{tabular}


TABLE VI.

Potentids for $\mathrm{Ne}+\mathrm{X}$ s Sytem

\begin{tabular}{|c|c|c|c|c|c|c|c|c|c|}
\hline System & No. & Potential Form & $e(* K)$ & $r_{m}(k)$ & $\mathbf{a}$ & $\beta$ & $\sigma_{12}$ & Roforence & $\begin{array}{l}\text { Standard deviation of } \\
\text { 2nd virial cooff fitting }\end{array}$ \\
\hline $\mathrm{Ne}+\mathrm{Xe}$ & $\begin{array}{l}1 \\
2 \\
3 \\
4 \\
5 \\
6 \\
7\end{array}$ & $\begin{array}{l}M S V \\
U(20,6) \\
\text { Exp-6 } \\
\text { Exp-6 } \\
\text { Exp-6 } \\
U(12,6) \\
U(12,6)\end{array}$ & $\begin{array}{l}74.98 \\
75.33 \pm 3.62 \\
61.9 \pm 5.0 \\
68.7 \pm 5.5 \\
73 . \pm 4 . \\
69.1 \pm 6.0 \\
78 . \pm 4\end{array}$ & $\begin{array}{l}3.745 \\
3.80 \pm 01 \\
4.01 \pm 04 \\
3.91 \pm .04 \\
3.87 \pm .04 \\
3.88 \pm .04\end{array}$ & $\begin{array}{l}14 \\
15 \\
15.5\end{array}$ & 7.9 & $\begin{array}{l}3.42 \\
3.48=.04 \\
3.46 \pm .03\end{array}$ & $\begin{array}{l}\text { this work } \\
1 \\
18 \\
18 \\
20 \\
18 \\
28\end{array}$ & 0.64 \\
\hline
\end{tabular}


TÁTiE VI"

DIFFUSION COEFFICIENTS $\left(\mathrm{cm}^{2} \mathrm{sec}^{-1}\right)$

\begin{tabular}{|c|c|c|c|c|c|c|c|c|c|}
\hline \multirow[b]{2}{*}{$T(K)$} & \multicolumn{3}{|c|}{ NEON - ARGON } & \multicolumn{3}{|c|}{ NEON - KRYPION } & \multicolumn{2}{|c|}{ NEON - XENON } & \multirow[b]{2}{*}{$\begin{array}{c}\% \\
\text { Difference }\end{array}$} \\
\hline & Calculated & Measured ${ }^{a}$ & $\begin{array}{c}\% \\
\text { Difference }\end{array}$ & Calculated & Meosured & $\begin{array}{c}\% \\
\text { Difference }\end{array}$ & Colculated & Measured $^{\circ}$ & \\
\hline 300 & .318 & .323 & -1.5 & .262 & .262 & 0.0 & .220 & .219 & 0.5 \\
\hline 350 & .415 & .421 & -1.4 & .342 & .342 & 0.0 & .286 & .286 & 0.0 \\
\hline 400 & .521 & .525 & $-0 . e$ & .429 & .431 & -0.5 & .358 & .359 & -0.3 \\
\hline 450 & .637 & .635 & 0.3 & .525 & .526 & -0.2 & .436 & .440 & -0.9 \\
\hline 500 & .761 & .765 & -0.5 & .627 & .627 & 0.0 & .520 & .523 & -0.6 \\
\hline 550 & .895 & .892 & 0.3 & .737 & .737 & 0.0 & .610 & 615 & -0.8 \\
\hline 600 & 1.037 & 1.045 & -0.8 & .853 & .850 & 0.4 & .705 & .710 & -0.7 \\
\hline 700 & 1.345 & 1.355 & -0.7 & I. 106 & 1.098 & 0.7 & .911 & .920 & -1.0 \\
\hline 800 & 1.685 & 1.675 & 0.6 & 1,384 & 1.38 & 0.4 & 1.14 & 1. 16 & -0.2 \\
\hline 1000 & 2,453 & 2.43 & 0.9 & 2.01 & 2.01 & 0.0 & 1.65 & 1.68 & -1.8 \\
\hline 1200 & 3,332 & 3.31 & 0,7 & 2.73 & 2.71 & 0.7 & 2,23 & 2.26 & -1.3 \\
\hline 1400 & 4.317 & 4.24 & 1.8 & 3.54 & 3.51 & 0.9 & 2.87 & $\because .92$ & -1.7 \\
\hline
\end{tabular}

a Measuremente of Hogervort, Ref. 28. 


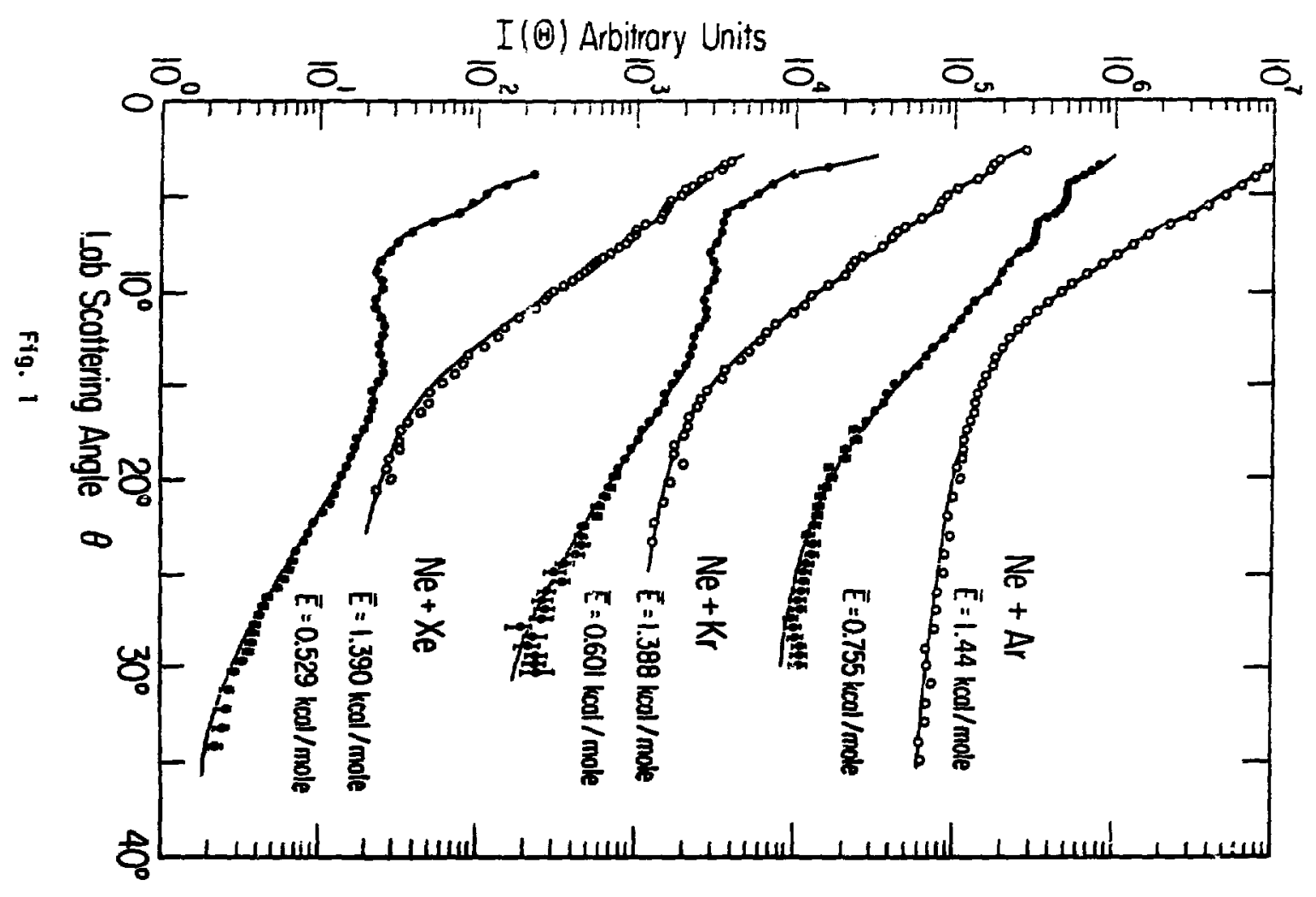

$\frac{1}{0}$ 


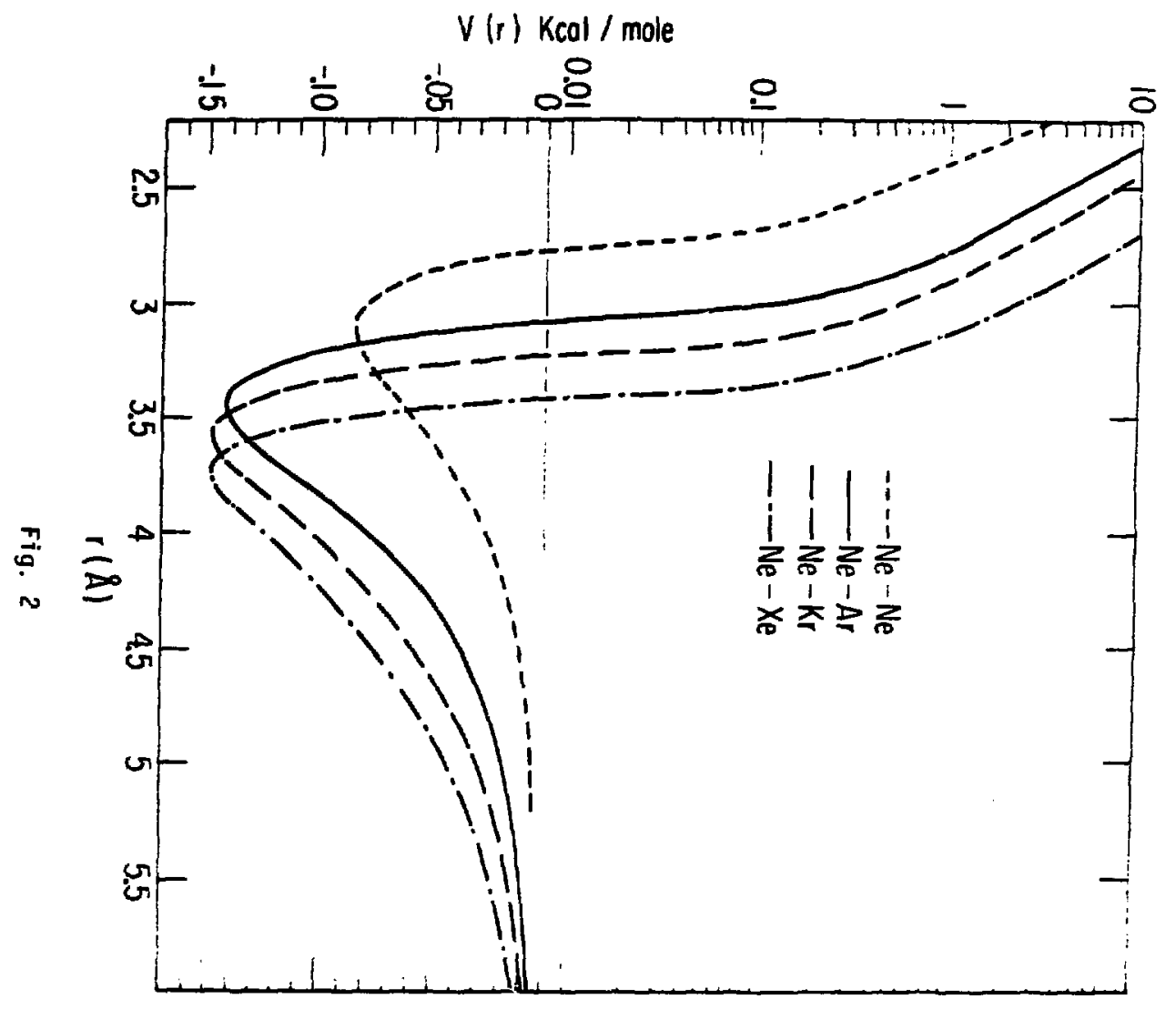




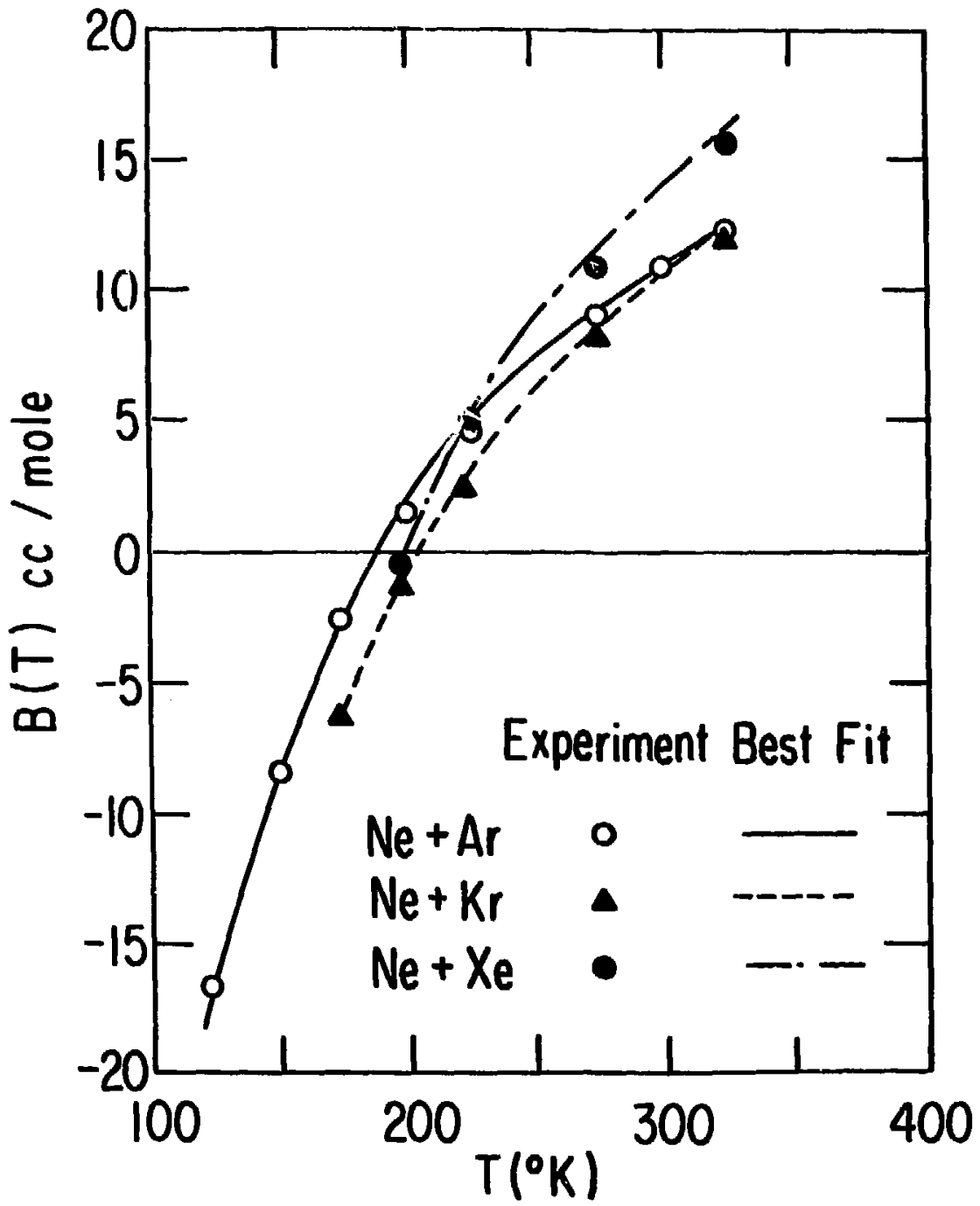

Fig. 3 


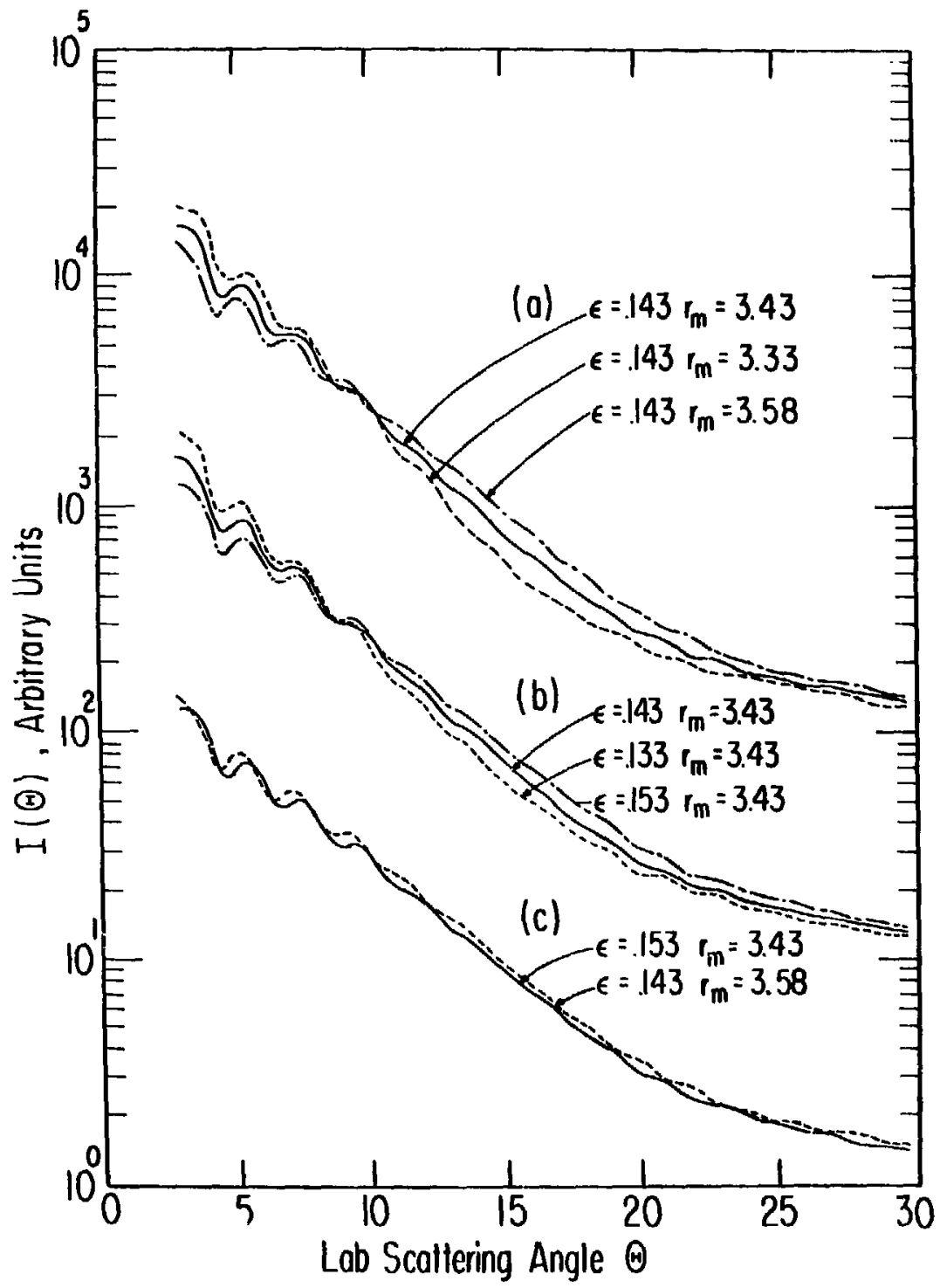

Fig. 4 


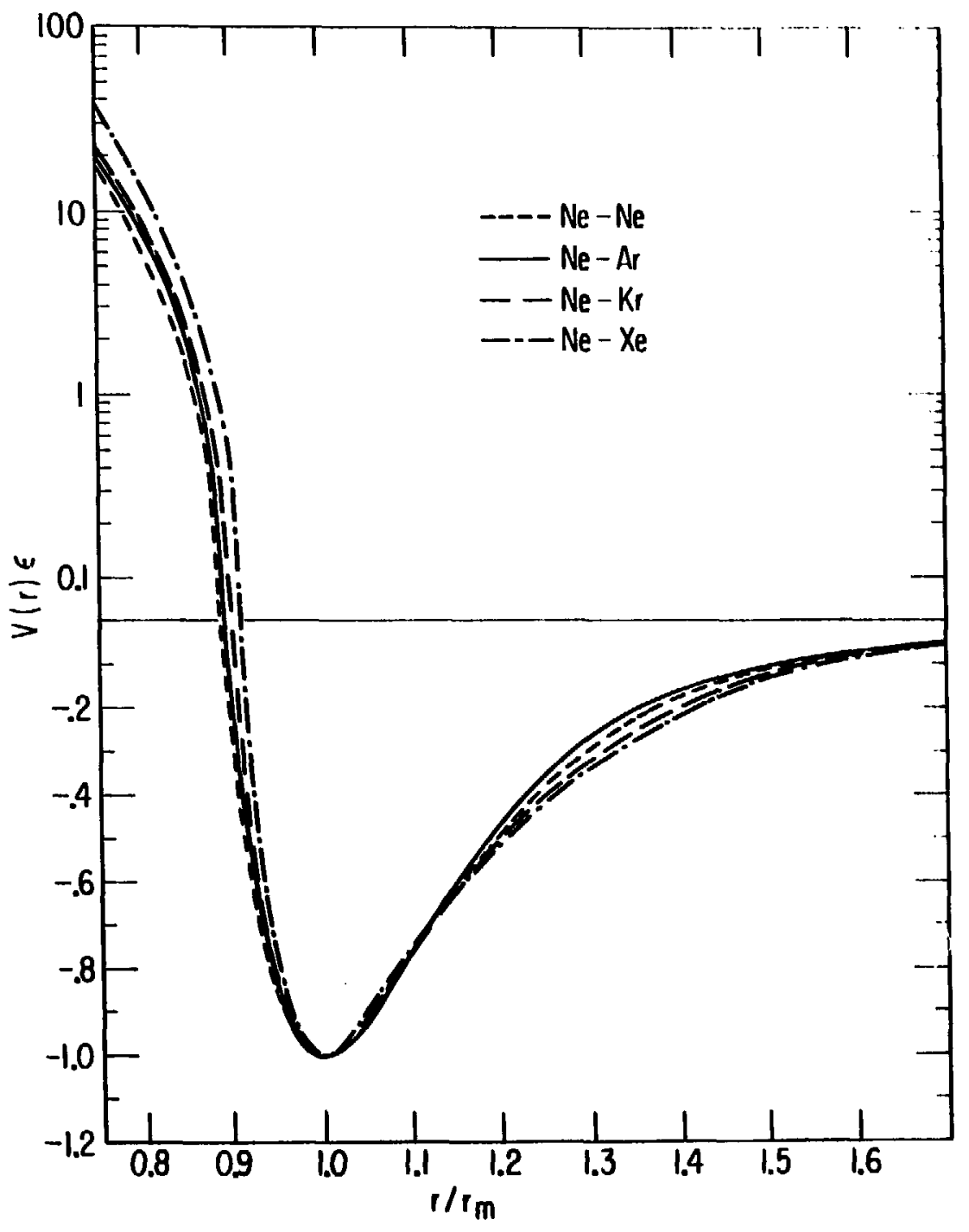

Fig. 5 


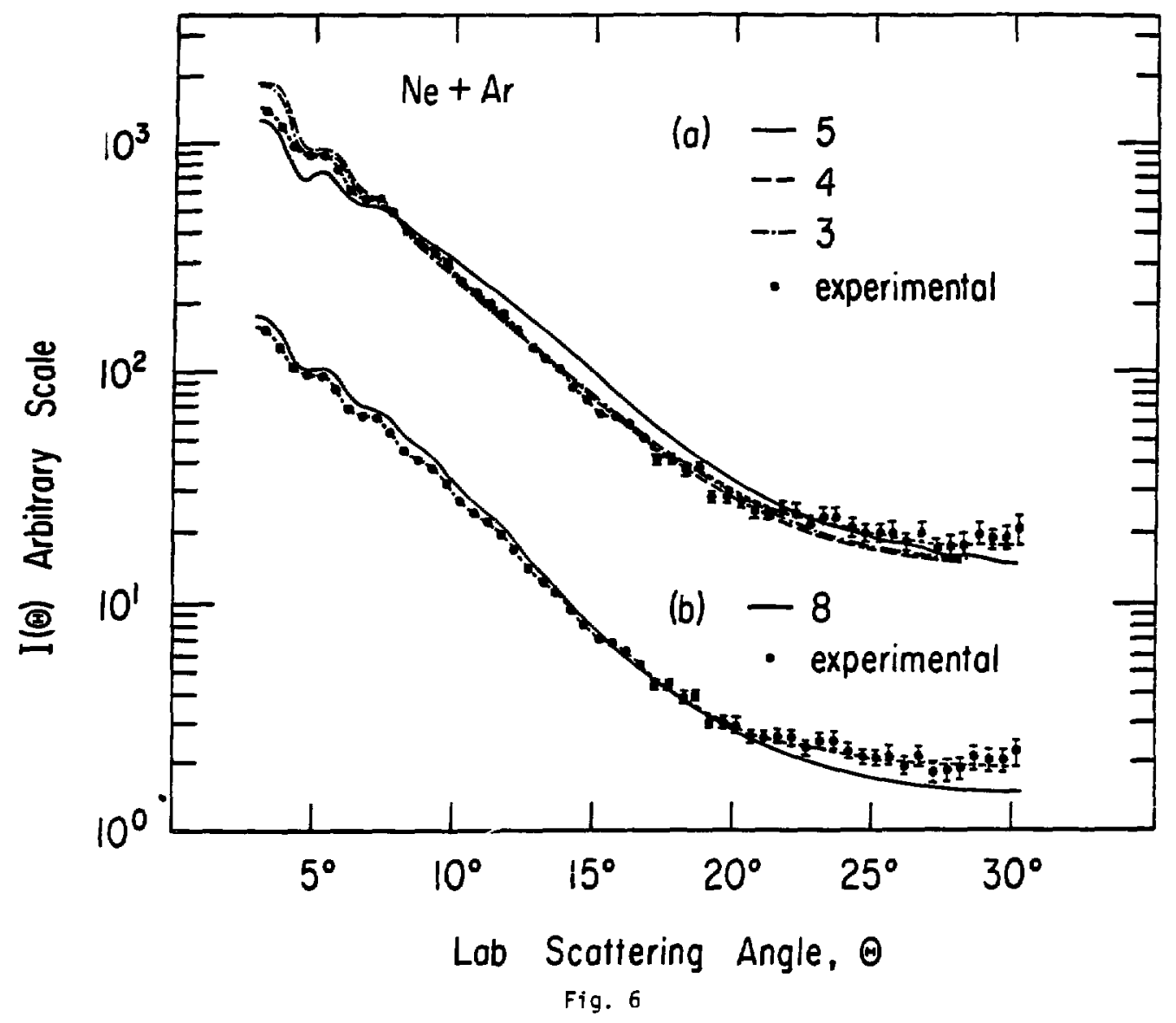




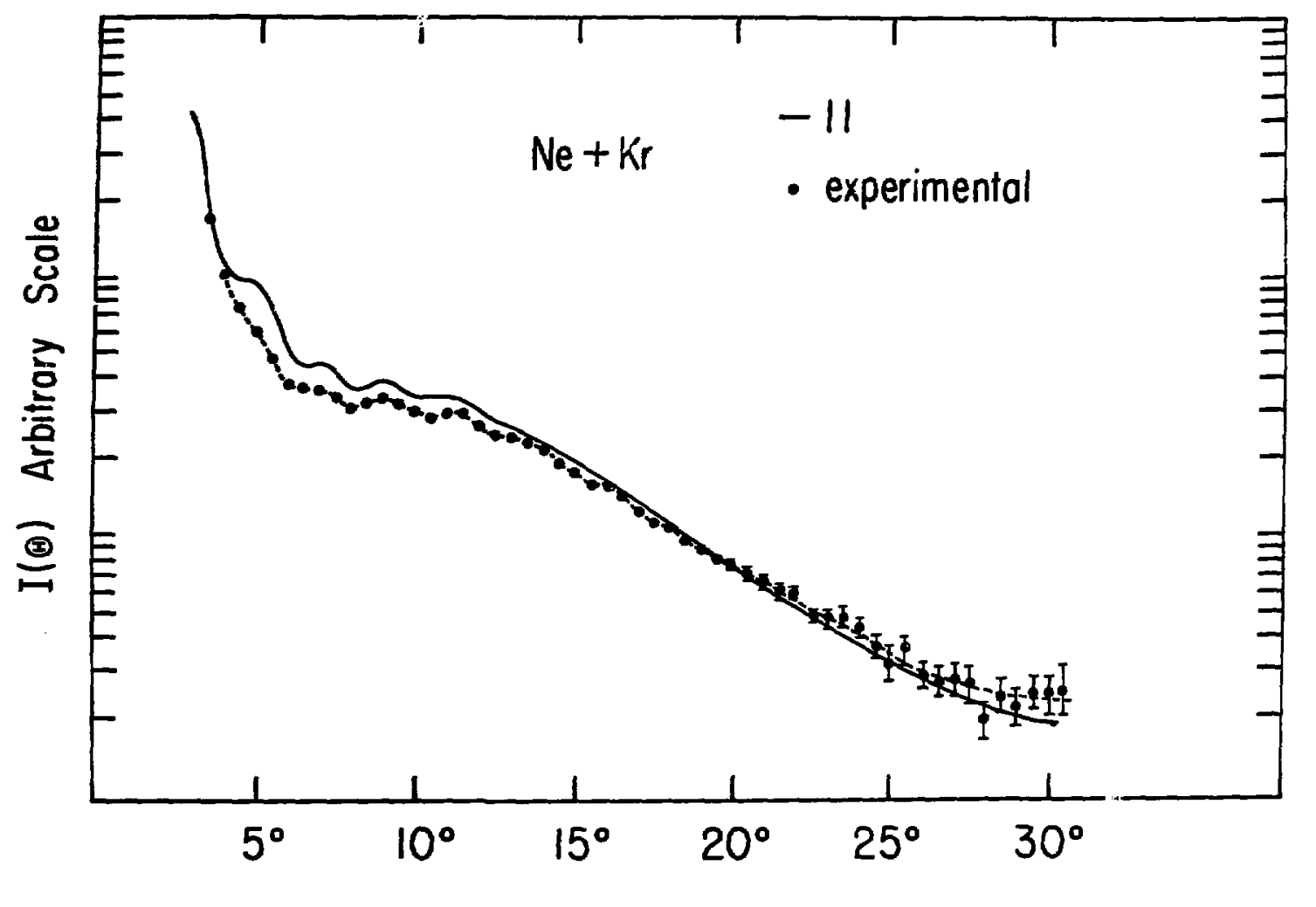

Lab Scattering Angle, $\Theta$

Fig. 7 


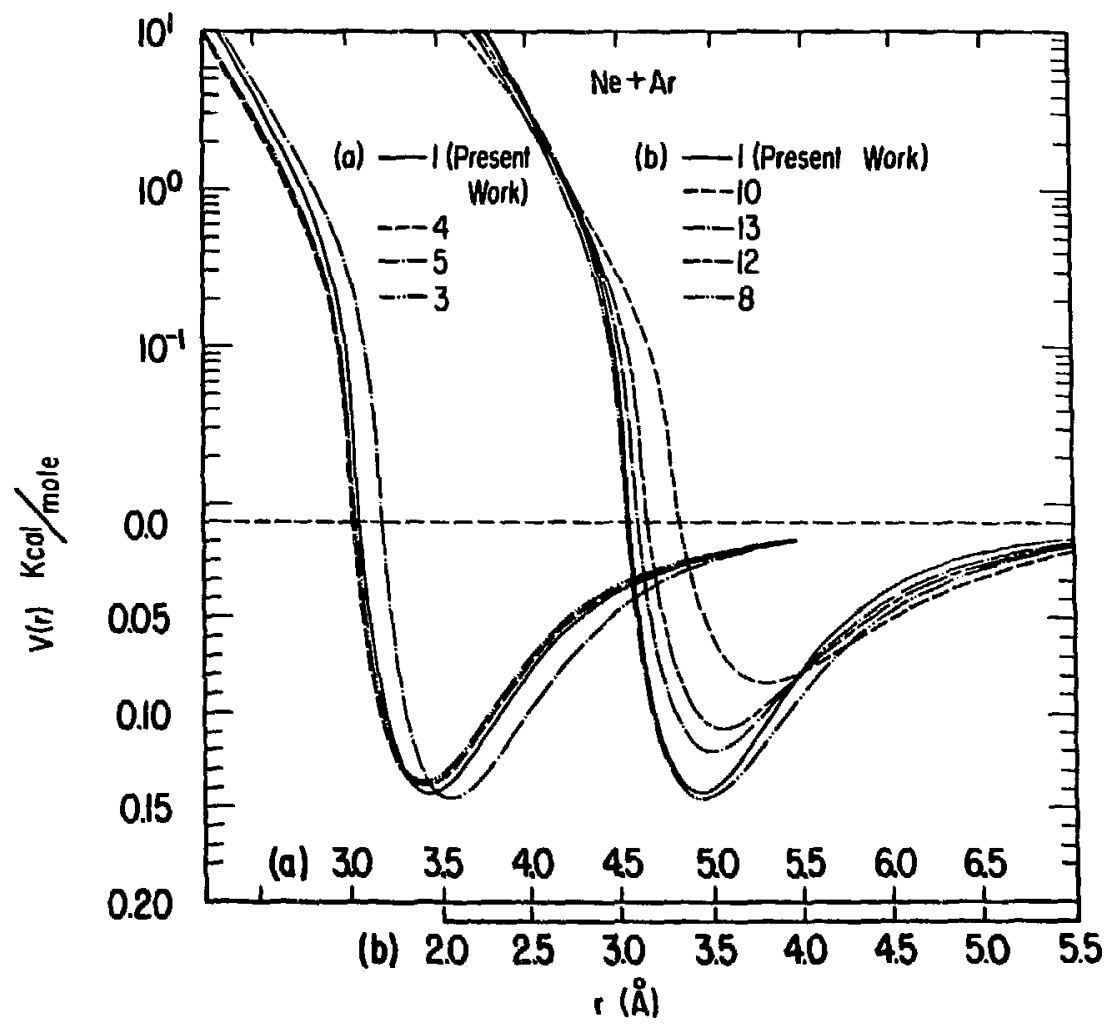

Fig. 8 


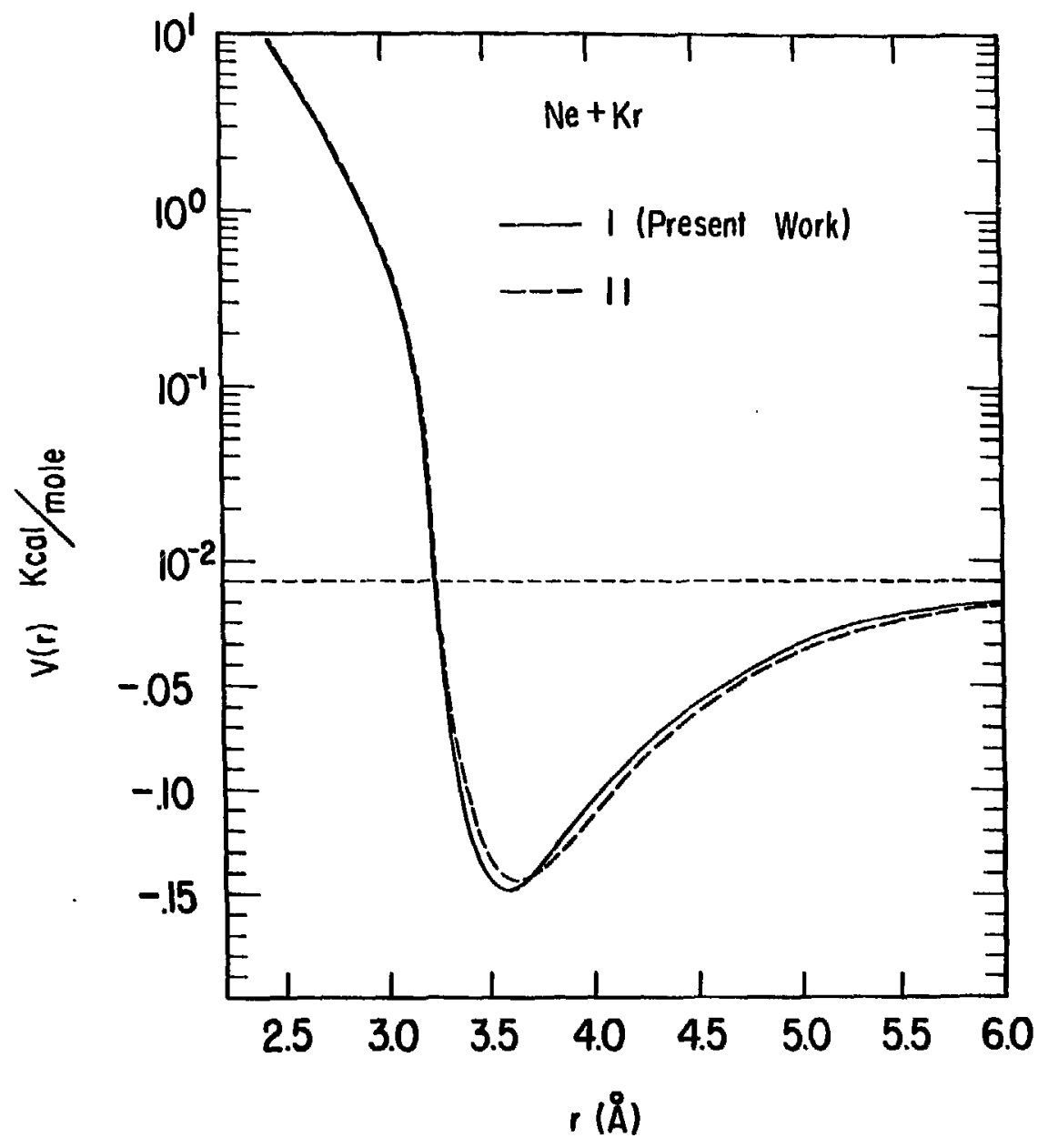

Fig. 9 


\section{APPENDICES}

A. Mechanical \& Vacuum Layout of the Photoionization Apparatus

Figure 1 and Fig. 2 show the detailed assembly diagrams of the photoionization apparatus in plane view and vertical view respectively. The arrangement and operation of the apparatus has been described briefly in the experimental part of chapter three. It is the purpose of this appendix to give a more detailed description on the differential pumping system for the monochromator.

The pumping arrangement for the monochromator is shown in Fig. 3. In order to excite the He Hopfield continuum, the lamp is at a steady pressure $P_{1}=100$ torr (He). If the area of the optical entrance slit between the lamp and the first differential pumping chamber is 10011 (width) $\times 0.5 \mathrm{~cm}$ (height) $=0.005 \mathrm{~cm}^{2}$, this corresponds to a conductance for He of $c_{1}=0.225 \ell / \mathrm{sec}$. The throughput of He from the lainp to the first differential pumping chamber is

$$
\begin{aligned}
Q_{1} & =\left(P_{1}-P_{2}\right)(0.225 \ell / \mathrm{s}): P_{1}(0.225 \ell / \mathrm{s}) \\
& =45(\text { torr } \cdot l) / \mathrm{sec} .
\end{aligned}
$$

The Roots pump has a pumping speed $S_{2}=300$ c.f.m. $=141 \ell$, sec and this gives a steady pressure

$$
P_{2}=\frac{45}{141} \text { torr }=0.32 \text { torr. }
$$

The area of slit 2 is $0.183 \mathrm{~cm}$ (width) $\times 0.498$ (height) $=0.090 \mathrm{cml}^{2}$, which corresponds to a conductance for He of $C_{2}=4.05 \mathrm{l} / \mathrm{sec}$. The second differential pumping region is pumped by an ejector pump which has a pumping speed of $S_{3}=300 \mathrm{l} / \mathrm{sec}$. The steady pressure is 
$-140-$

$$
P_{3}=\frac{(4.05)(0.32)}{300}=4.3 \times 10^{-2} \text { torr. }
$$

The area of the third slit is $0.35 \mathrm{~cm}$ (width) $\times 0.584 \mathrm{~cm}$ (height) $=0.204 \mathrm{~cm}^{2}$, and this gives a conductance for He of $c_{3}=9.2 \mathrm{l} / \mathrm{sec}$.

The pumping speed of the $6^{\prime \prime}$ diffusion pump for He is $* 2500 \mathrm{l} / \mathrm{sec}$.

Thus, the pressure in the main chamber of the monochromator is

$$
P_{3}=\frac{9.2 \times 4.3 \times 10^{-2}}{2500}=1.5 \times 10^{-4} \text { torr. }
$$




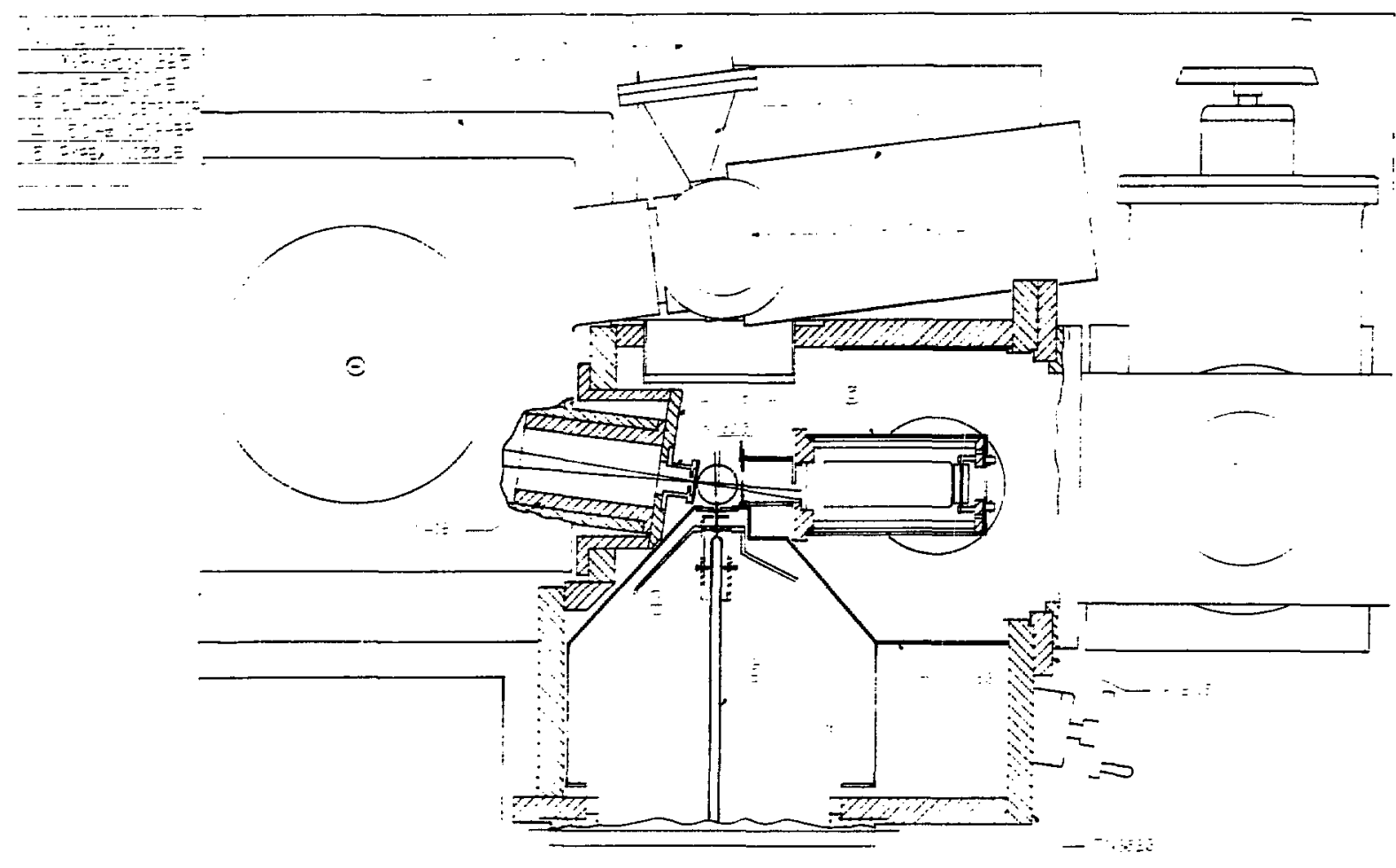

$\frac{1}{1}$

Fig. 1. Assembly diagram of the photoionization apparatus (Plane view) 


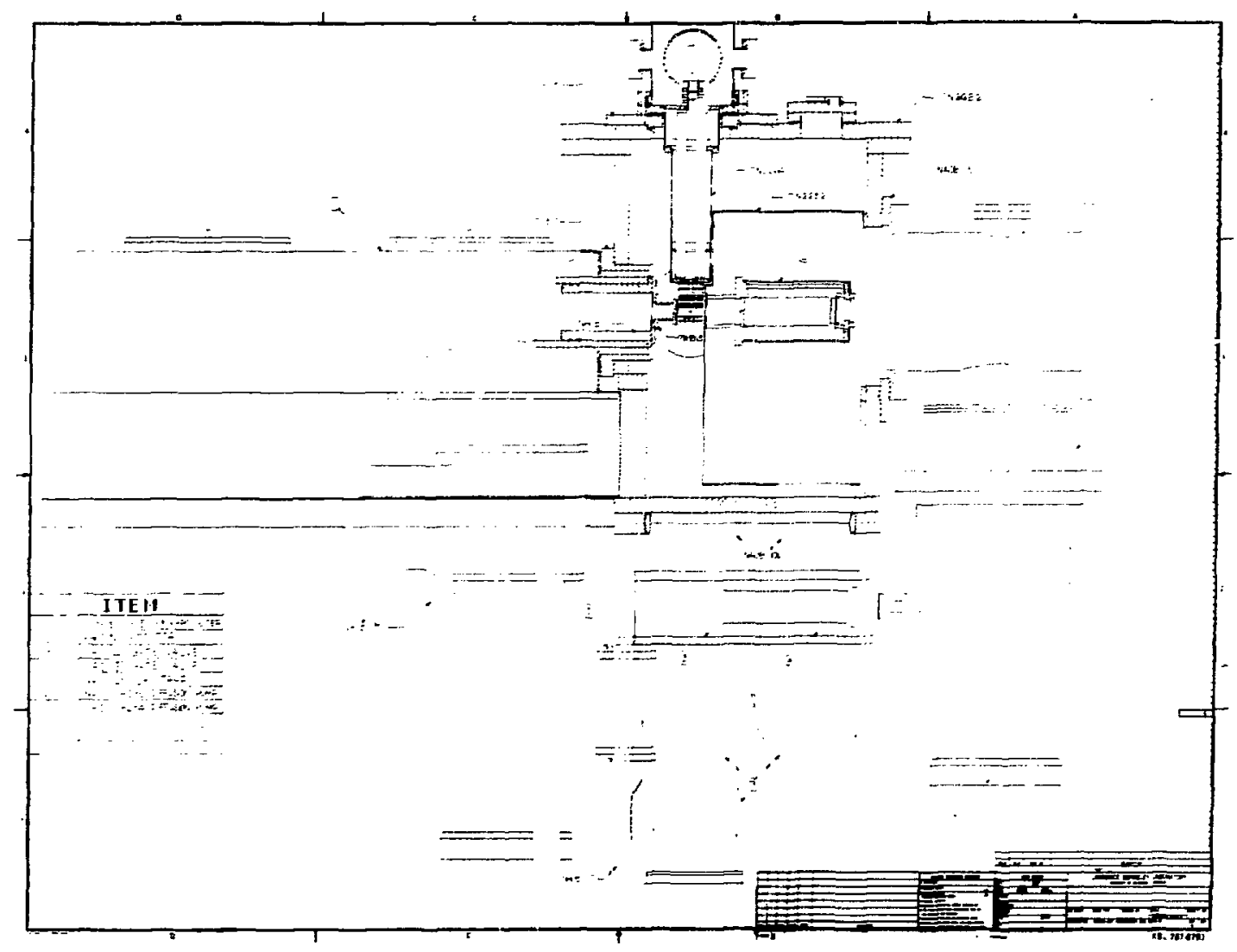

Fig. 2. Assembly diagram of the photoionization apparatus (vertical view). 

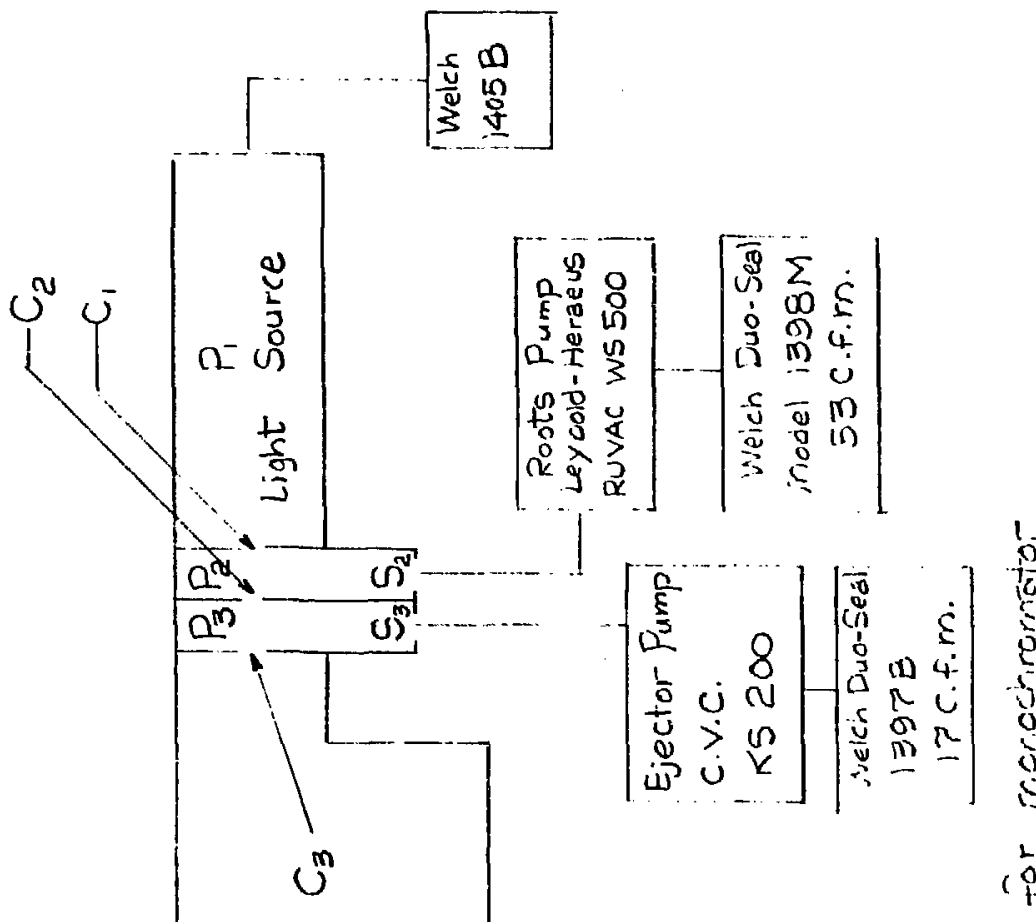


\section{B. Molecular Beam Production System}

The pumping and mechanical arrangement for the molecular beam production system is shown in Fig. 1. The exhausted chamber is pumped by a 10" diffusion pump (Varian HS 10) which has a nominal pumping speed of $S_{1} \approx 4000 \mathrm{2} / \mathrm{sec}$, and the buffer chamber is pumped by a $4^{\prime \prime}$ diffusion pump (Varian VHS4) with an effective pumping speed of about $S_{2}=550 \mathrm{l} / \mathrm{s}$ (used with a gate valve). The main chamber is pumped by two 4" and one 6" diffusion pumps which is estimated to have an effective pumping speed of $\mathrm{S}_{3}=2500 \mathrm{\ell} / \mathrm{sec}$.

Using argon gas as an example, a source pressure of 760 torr at room temperature $\left(298^{\circ} \mathrm{K}\right)$ corresponds to the mass flow through the nozzle and is described by

$$
\begin{aligned}
\dot{m} & =14.3 D_{0}^{2} \cdot p_{0} \frac{\text { torr } \cdot 1 i t}{\mathrm{sec}} \\
& =(14.3)(0.0127)^{2} \cdot(760) \\
& =1.75 \frac{\text { torr } \cdot 1 \mathrm{it}}{\mathrm{sec}}
\end{aligned}
$$

where $D_{0}=0.0127 \mathrm{~cm}$ is the diameter of the nozzle

$$
P_{0}=760 \text { torr is the nozzle stagnation pressure. }
$$

Hence the normal pressure of the exhausted chamber is

$$
\begin{aligned}
P_{1} & =\left(1.75 \frac{\text { torr. } 1 \text { itt }}{\sec }\right) / 4000 \mathrm{lit} / \mathrm{sec} \\
& =4.4 \times 10^{-4} \mathrm{torr}
\end{aligned}
$$

The solfd angle subtended by a $0.0635 \mathrm{~cm}$ dlameter skimmer is

$$
\Delta \Omega=\frac{\left(\frac{\pi}{4}\right)(0.0635)^{2}}{(0.5)^{2}}=0.01268 \text { sterad. }
$$


For an isentropic expansion, the number density on the beam axis $n(x)$ is approximated by

$$
n=0.161 \times n_{0} \times P_{0}\left(\frac{x}{D_{0}}\right)^{-2}
$$

At STP, the number density of one torr is

$$
n_{0}=3.24 \times 10^{16} \text { atom/c.c. }
$$

The number density at the skimmer entrance is

$$
\begin{aligned}
n & =0.161 \times 3.24 \times 10^{16} \times 760\left(\frac{0.127}{5}\right)^{2} \\
& =18 \times 10^{14} \text { atom/c.c. }
\end{aligned}
$$

The mean velocity of argon at room temperature $\left(298^{\circ} \mathrm{K}\right)$ is

$$
v=5 \times 10^{4} \mathrm{~cm} / \mathrm{sec}
$$

which gives a beam flux at the skimmer entrance to be

$$
n \times v=5 \times 10^{4} \times 18 \times 10^{14}=9 \times 10^{19} \mathrm{atom} / \mathrm{cm}^{2} \cdot \mathrm{sec}
$$

The number of atoms entering the differential punping chamber is

$$
9 \times 10^{19} \times \frac{\pi}{4}(0.0635)^{2} \text { atoms } / \mathrm{sec}
$$

This corresponds to a pressure volume product of

$$
\begin{aligned}
& =\left(3 \times 10^{17} \text { atom/c.c. sec }\right) /\left(3.24 \times 10^{19} \text { atonl } / \text { tor } \cdot \ell\right) \\
& =9.3 \times 10^{-3} \text { torr } \cdot 1 \mathrm{it} / \mathrm{sec}
\end{aligned}
$$

This gives a normal pressure, $P_{2}$, for the buffer chamber of

$$
P_{2}=\frac{9.3 \times 10^{-3}}{550 \ell / \mathrm{sec}}=1.7 \times 10^{-5} \text { torr }
$$

The area of defining slit in the diffarential punping wall is

$$
\begin{aligned}
=\text { width } \times \text { height } & =0.1 \times 0.323 \\
& =0.0323 \mathrm{clll}^{2}
\end{aligned}
$$

Thus, the pressure in the nain chamber is

$$
P_{3}=5 \times 10^{-7} \text { torr. }
$$


The number density of the beam at the collision center is equal to $1.1 \times 10^{13}$ atom/c.c. $\approx 3 \times 10^{-4}$ torr/c.c. at S.T.P.

Figure 2 shows the plot of the beam intensity of acetylene $\left(\mathrm{C}_{2} \mathrm{H}_{2}\right)$ at the collision center versus the nozzle stagnation pressure as probed with an electron gun. The solid curve is the experimental curve and the dashed line is the predicted curve if we expect a linear increase in beam intensity as a function of stagnation pressure. 


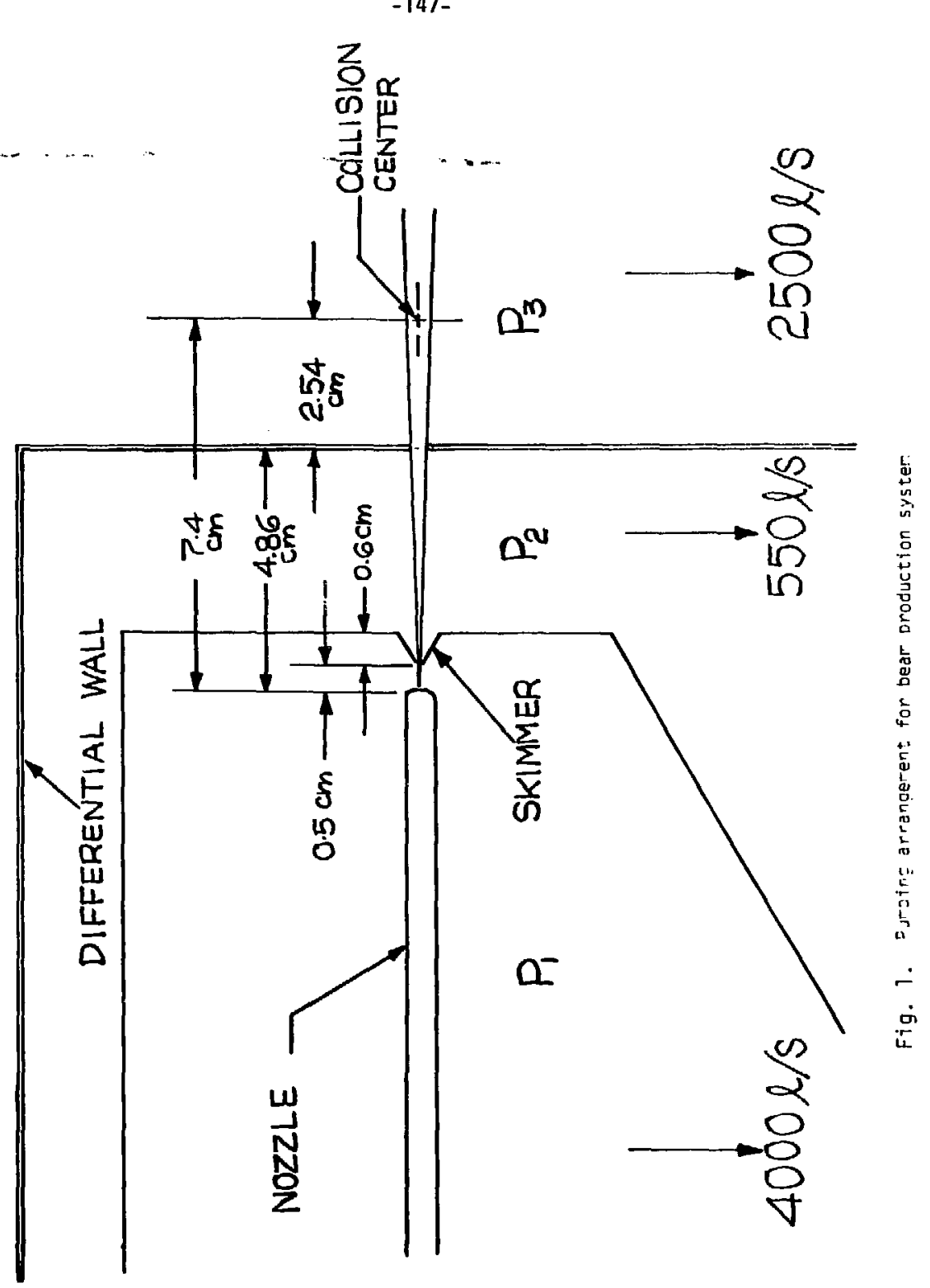




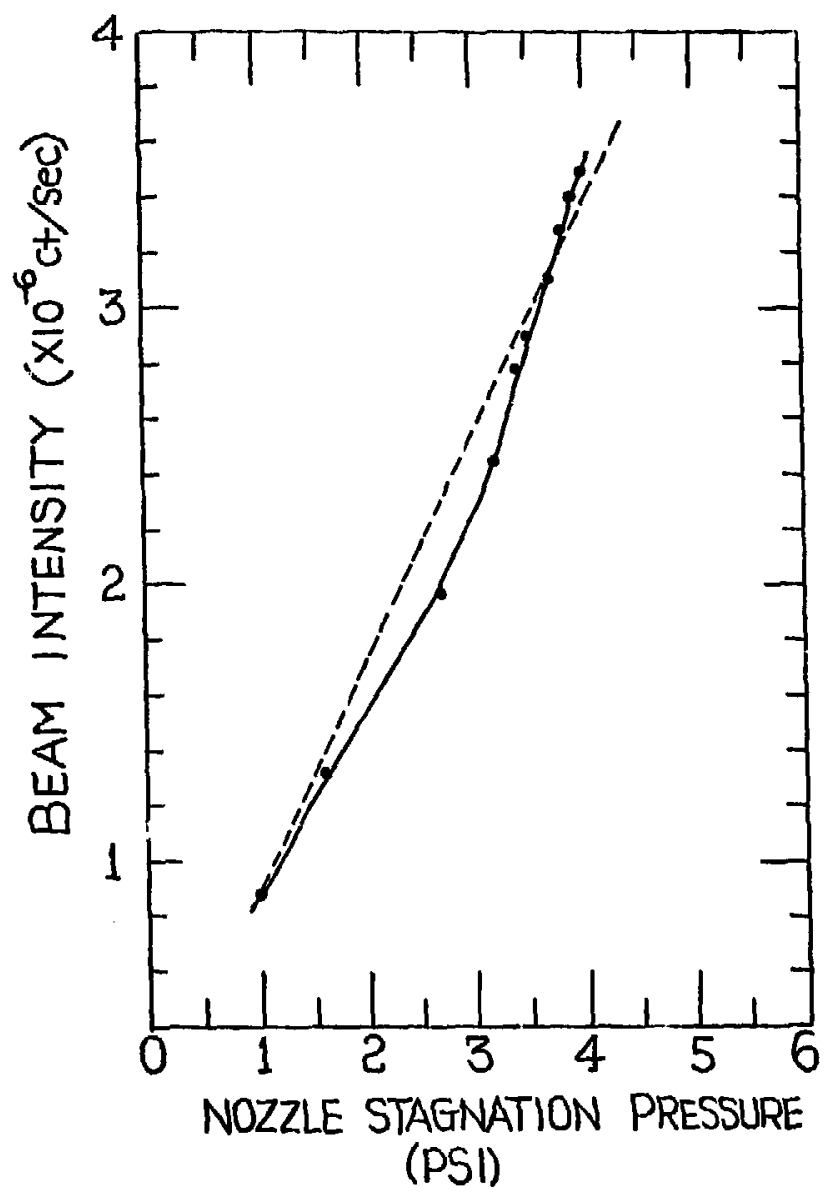

Fig. 2. Beam intensity of acetylene $\left(\mathrm{C}_{2} \mathrm{H}_{2}\right)$ at the collision center as a function of nozzle stagnation pressure 


\section{Vacuum Ultraviolet Light Source}

There are many light sources which have been designed and used for vacuum radiation studies. The choice of source depends on the application. A line spectrum is often more intense than a continuum source. For photoionization cross section measurements studied as a function of wavelength, it is preferable to use a continuum light source. Furthermore, for the photoionization studies in this research, the photon energies used lie between $6 \mathrm{eV}$ and $21 \mathrm{eV}$. The various types of continuum light sources which produce photon energies within this range have been discussed by Samson ${ }^{1}$ and described briefly in the following sections. I. (a) The Hydrogen Continuum and Hydrogen Many-Line Pseudocontinuum. The DC capillary discharge of $\mathrm{H}_{2}$ at low pressure (1-5 torr) produces an enission continuum which extends from $1600 \AA$ to $5000 \AA$. The sharp band structure of hydrogen starts at $1670 \AA$ and extends down to $850 \AA$. This has the appearance of a line spectrum and gives rise to the commonly used term "the many line molecular hydrogen pseudocontinuum spectrum." The spectral range with which we are concerned for our application is shown in Fig. 1. The range is mainly covered by the hydrogen many-line pseudocontinuum. At low discharge pressures, the atomic resonance lines are more prominent than the band structure. On the other hand, an increase in discharge pressure and a corresponding increase in power dissipation in the light source, enhance the light intensity of the band structure and the atonic resonance lines are suppressed. In addition, the light intensity increases nearly linearly with power dissipation in the discharge lamp and the length of discharge capillary. 
Th1s is one of the most cormonly used laboratory light sources in the wavelength region from $950 \AA$ to $2000 \AA$. However, due to the sharp-line band structure, it is not suftable for high resolution studies.

(b) The He, Ne, Ar, $\mathrm{Kr}$ and Xe Hopfield Continua

Hopfield $d^{2-4}$ first dtscovered the continuous emission spectrum of helium extending from $600 \AA$ to $1000 \AA$. The continuum has been studied extensively by Tanaka and Huffman. $5-10$ Huffman has observed that the continuum is emitted as an afterglow. In addition, it was found that helfum continuum is best produced by a condensed spark discharge which produces more fonization. The following chain of reactions is suggested as a mechanism operative in a spark discharge in helflun

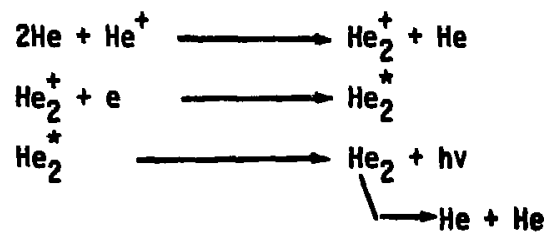

Thus the intensity of the continuum would be expected to increase with pressure until the competing processes become important. Another fact which suggests that the $\mathrm{He}^{+}$ion is the primary reactant is the necessity for extremely pure helium in producing the continuum. In a gas discharge, the energy is absorbed primarily by the species with the lowest ionization potential, and since He has the-greatest ionization potential of all the elements $(24.58 \mathrm{eV})$, any impurity will tend to decrease the number of $\mathrm{He}^{+}$lons. Tanaka et. al., 11 have attributed the origin of two main peaks near $810 \AA$ and $680 \AA$ to the transitions $A^{1} \Sigma_{u}^{+}+x^{1} \Sigma_{g}^{+}$and $D^{1} \Sigma_{u}^{+}+x^{1} \Sigma_{g}^{+}$respectively, as shown in Fig. 3 . The helium continuum is shown in Fig. 4. 
Similar emission continua for $\mathrm{Ne}, \mathrm{Kr}$ and $\mathrm{Xe}$ have been found by Takamine ${ }^{12}$ and Tanaka et. al. 13-15 The intensity distributions in the continuous emission spectra of $\mathrm{Ar}, \mathrm{Kr}$ and $\mathrm{Xe}$ as excited by a $125 \mathrm{~W}$ microwave generator are shown in Fig. 2. These three continua, nearly cover the same spectral region as the hydrogen many-line continuum. However, due to the high cost of $k r$ and $X_{e}$, the $k r$ and $X_{e}$ continua are seldanly used in Laboratory.

By combining the use of helium Hopfield continuum and the hydrogen many-line pseudocontinuum, we essentially have a continuous light source covering the wavelength range from $600 \AA$ to $2000 \AA(-6 \mathrm{eV}-21 \mathrm{eV})$. This is sufficient for the photoionization experiments of this research. II. Mechanical Construction and Operation of the Light Source The discharge lamp consists of a quartz capillary discharge tube and two aluminum electrodes. The discharge capillary is a $6 \mathrm{~mm}$ O.D. and 11 inches long quartz capillary which is surrounded by a water jacket. The two ends of the tube are vacuum sealed to two water cooled a luninum electrodes by two cajoins. The assenbly of the discharge lamp is shown in Fig. 7. The anode (which closes to the differential pumping arm of the monochromator) is normally grounded. During the operation of the lamp, gas ( $\mathrm{He}$ or $\mathrm{H}_{2}$ ) is being fed into the lamp from the front anode and continuously pumped out from the cathode by a small mechanical pump. This prevents the optical entrance slit which is usually very narrow from being clogged up by sputtering material produced during the discharge. The schematic diagran showing the discharge gas regulated systell is shown in Fig. 5. 
To excite the hydrogen many-line pseudocontinuum, about 3-5 torr of $\mathrm{H}_{2}$ at a steady flow is maintained in the discharge lamp. The hydrogen gas used is the ultra-pure grade ( $99.999 \%$ purity) obtained from Matheson. A 1:1 ratio mixture of hydrogen and deuterium has also been used. Due to the difference in zero point energy of $\mathrm{H}_{2}, \mathrm{D}_{2}$ and $\mathrm{HD}, \mathrm{a}$ better continuum is to be anticipated. The lamp is powered by a McPherson Model 740 d.c. power supply as shown in Fig. 6(a). The typical output spectrum obtained is shown in Fig. 1 .

In order to excite the He Hopfield continuum, a high power pulser is used to pulse the discharge lamp. The helfum used is also the ultrahigh purity grade ( $99.999 \%$ minimum purity) obtained from Matheson. The electronic schematic block diagram is shown in Fig. 6(b). The Velonex 360 is used as a pulse oscillator. The frequency of this unit is continuously tunable from $-1 \mathrm{~Hz}$ to $0.3 \mathrm{MHz}$ and the pulse width can be changed continuously from $0.05 \mu \mathrm{sec}$ to $3 \mathrm{~m}$ sec with a maximum duty factor of 1.5\%. The pulse generated by the Velonex 360 is further amplifieú ioy a pulse amplifter (LBL $17 \times 4504 \mathrm{~W}-2$ ) which is powered by a $15 \mathrm{kV}, 500 \mathrm{nd}$ d.c. power supply. The output is then fed to the cathode of the discharge lamp. As expected, it is found that the light intensity increases with the discharge He pressure. With a $100 \mu$ optical entrance sift and the present differentiai pumping system, the maximum discharge He pressure allowed is -100 torr. The maximum photon intensity was obtained when the repetitional rate of the pulse Is $100 \mathrm{KHz}$, the pulse width is about $0.5 \mu \mathrm{sec}$ and the d.c. power supply is set at $6 \mathrm{kV}, 300 \mathrm{~mA}$. The typical He Hopfield emission 
$-153-$

spectrum observed is similar to that shown in Fig. 4. With the discharge conditions mentioned above, and the present monochromator system, about $10^{10}$ photon/sec $\AA$ at $800 \AA$ are obtained at the exit slit of the monochromator as estimated by a nickel photoelectric ce ll. 


\section{REFERENCES}

1. J.A.R. Sanson, "Techntques of Vacuum U1travtolet Spectroscopy" John WIley and Son, Inc., New York, 1967.

2. J. J. Hopfteld, Phys. Rev., 35, 1133 (1930).

3. J. J. Hopfield, Phys, Rev., 36, 784 (1930).

4. J. J. Hopfield, Astrophys, J., 72, 133 (1930).

5. R. E. Huffiman, Y. Tanaka, and J.C.L.C. Larrabee, App1. Optics 2. 617 (1963).

6. R. E. Huffman, J. C. Larrabee, Y. Tanaka and D. Chambers, J. Opt. Soc. Am., 55, 101 (1965).

7. R. E. Huffman, L. C. Larrabee and D. Chambers, Appl. Optics, 4, 1145 (1965).

8. R. E. Huffman, H. H. Hunt, Y. Tanaka and R. L. Novack, J. Opt. Sac. An., 51, 693 (1961).

9. R. E. Huffman, Y. Tanaka, and J. C. Larrabee, J. Opt. Soc. Am., 52. 851 (1962).

10. R. E. Huffman, J. C. Larrabee, and Y. Tanaka, Appl. Optics, 4, 1581 (1965).

11. Y. Tanaka, A. S. Jursa, and F. J. Leblanc, J. Opt. Soc. An., 48, 304 (1958).

12. T. Takamine, T. Suger, Y. Tanaka, and G. Imotant, Set, Papers Inst. Phys. Chem. Research (Tokyo), 35, 447 (1939).

13. Y. Tanaka, and M. Zelikoff, Phys. Rev., 93,933 (1954).

14. Y. Tanaka, and M. Zelikoff, J. Opt. Soc. Am., 44, 254 (1954).

15. Y. Tanaka, J. Opt. Soc. An., 45, 710 (1955).

16. P. G. Wfikinson and E. T. Bryam, App1. Optics, 4. 581 (1965). 


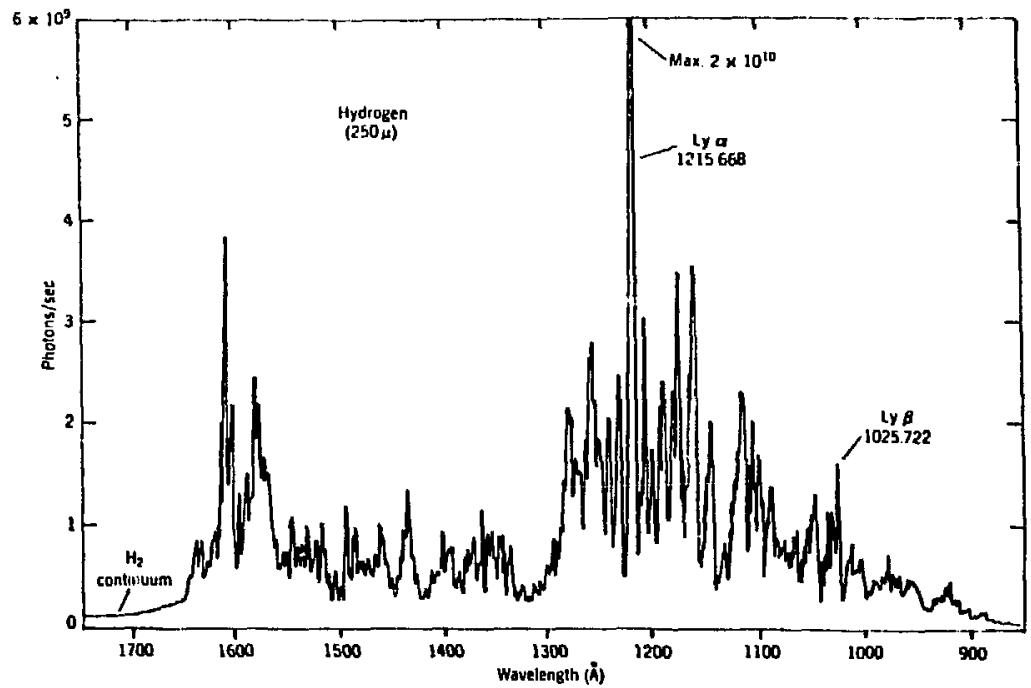

Fig. 1. H, specirum hetween 850 and 1750 A. Pressure, $250 \mu$; diseharge currem, $400 \mathrm{~mA}$. No ratiation is produced at wavelengitis shorter than $800 \AA$.

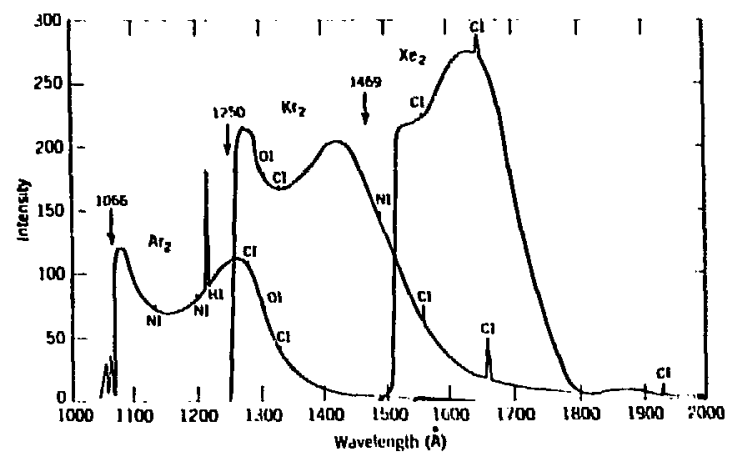

Fig. 2. Intensity distribution in the continuous emission spectrum of argon, kryphon, and xesum as excited by a 125.W nuicrowave penerator. Cias pressure was 200 torr.

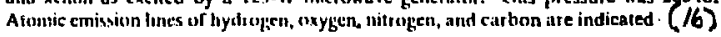




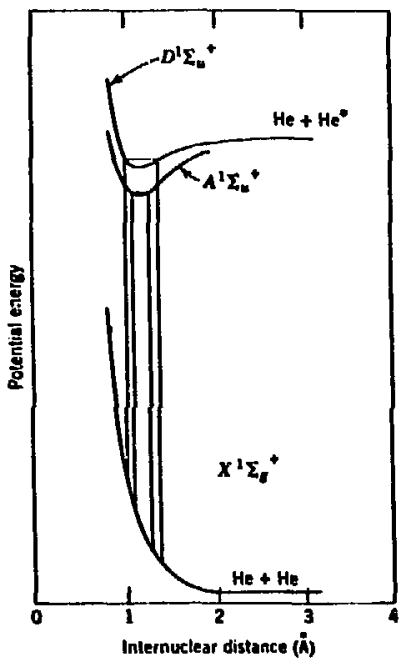

Fig. 3. Schematic diagram of the potential energy curves of $\mathbf{H e}$.

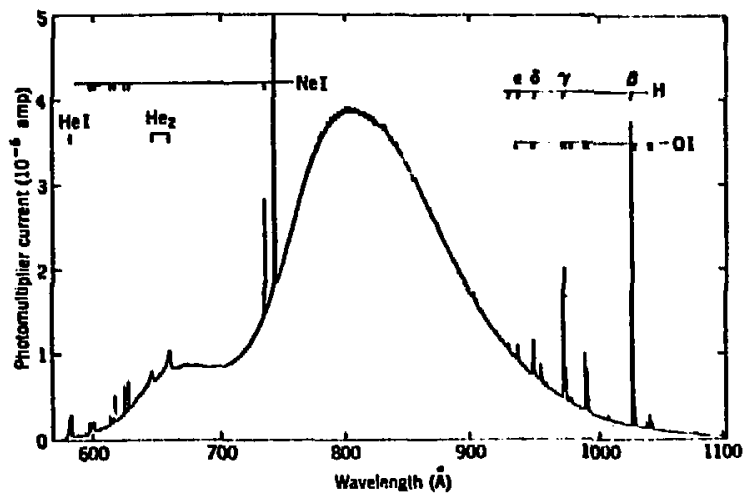

Fig. 4. Helium continuun using thyratron modulator. Conditions: sodium salicylate photomultiplier detector (EMI 95145) at $1630 \mathrm{~V}_{;} 0.5 \mathrm{~A}$ bandwidth using 100- $\mu$ slits; power supply at $116 \mathrm{~mA}$ and $10 \mathrm{kV}$; 5-kc sec pulse repetition frequescy; 44-mm IIg helium pressure; 1.0-sec time coustint (7) 


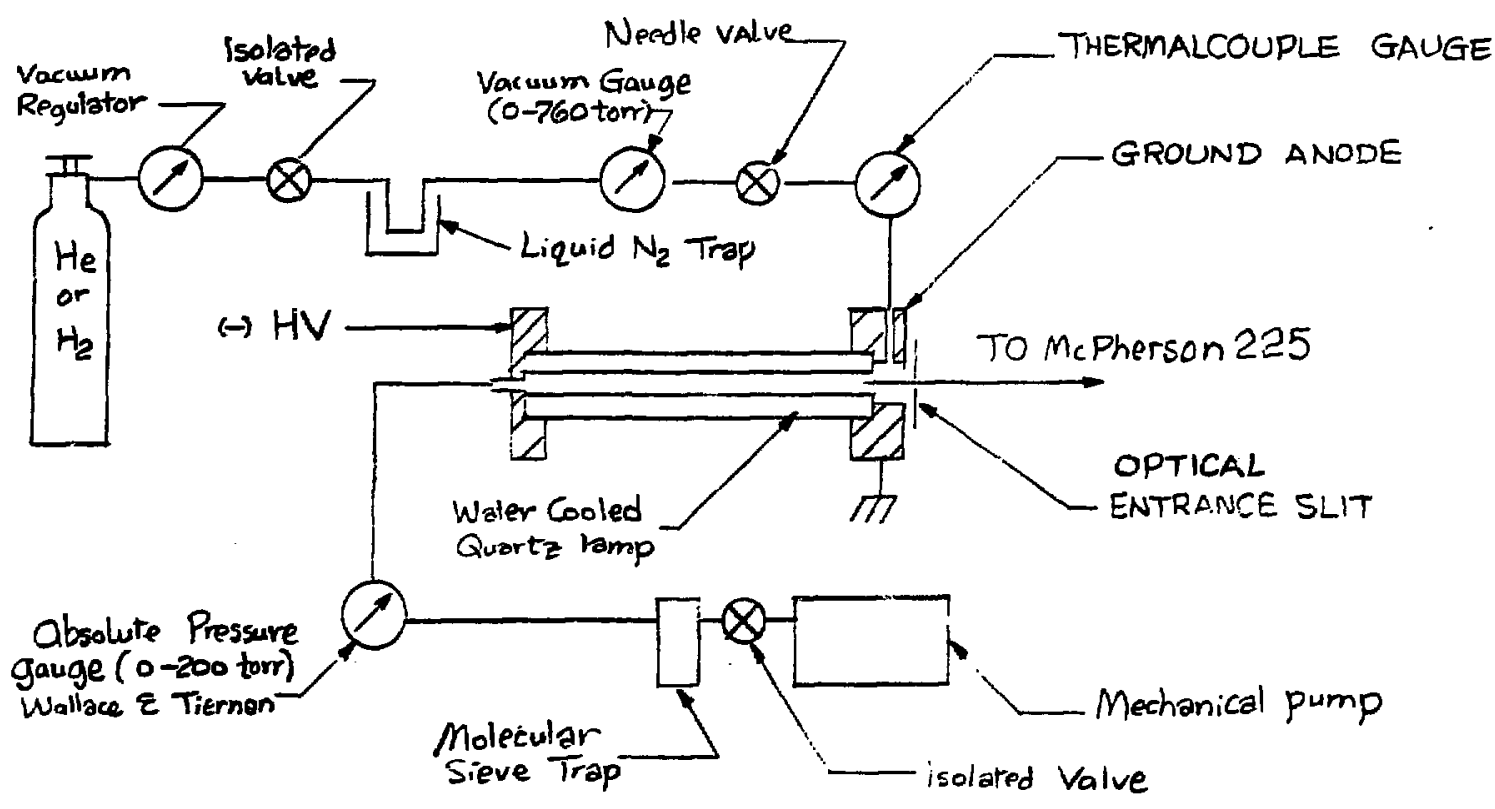

Fig. 5. Dischaige Gases regulated System 
MePherson

Madel P4O

(a)

5KV, IAmp

D.C. power supply

(-) HV To cothode of Discharge lamp

(b)
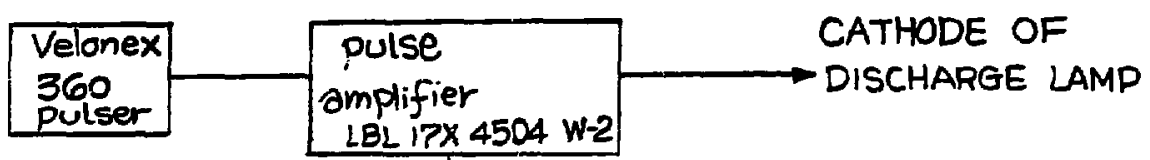

$\stackrel{\dot{1}}{\stackrel{1}{1}}$

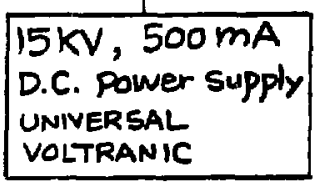

Fig. 6. Block assembly of discharge power supply. (a) Power supply to produce $\mathrm{H}_{2}$ pseudocontinuum.

(b) Power supply to produce $\mathrm{He}$ Hopfied continuum. 

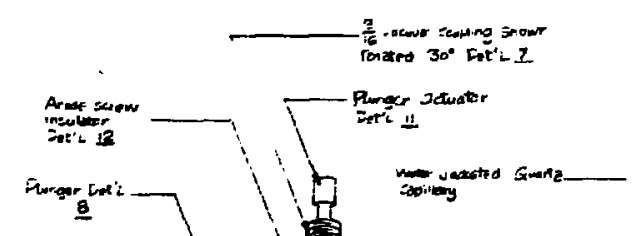

ard... verpling rot and

rg aft - g

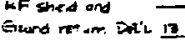

lifes zes

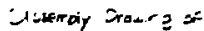
2 Juerjerz amf.

Foll Jeale

$1,-21-73$

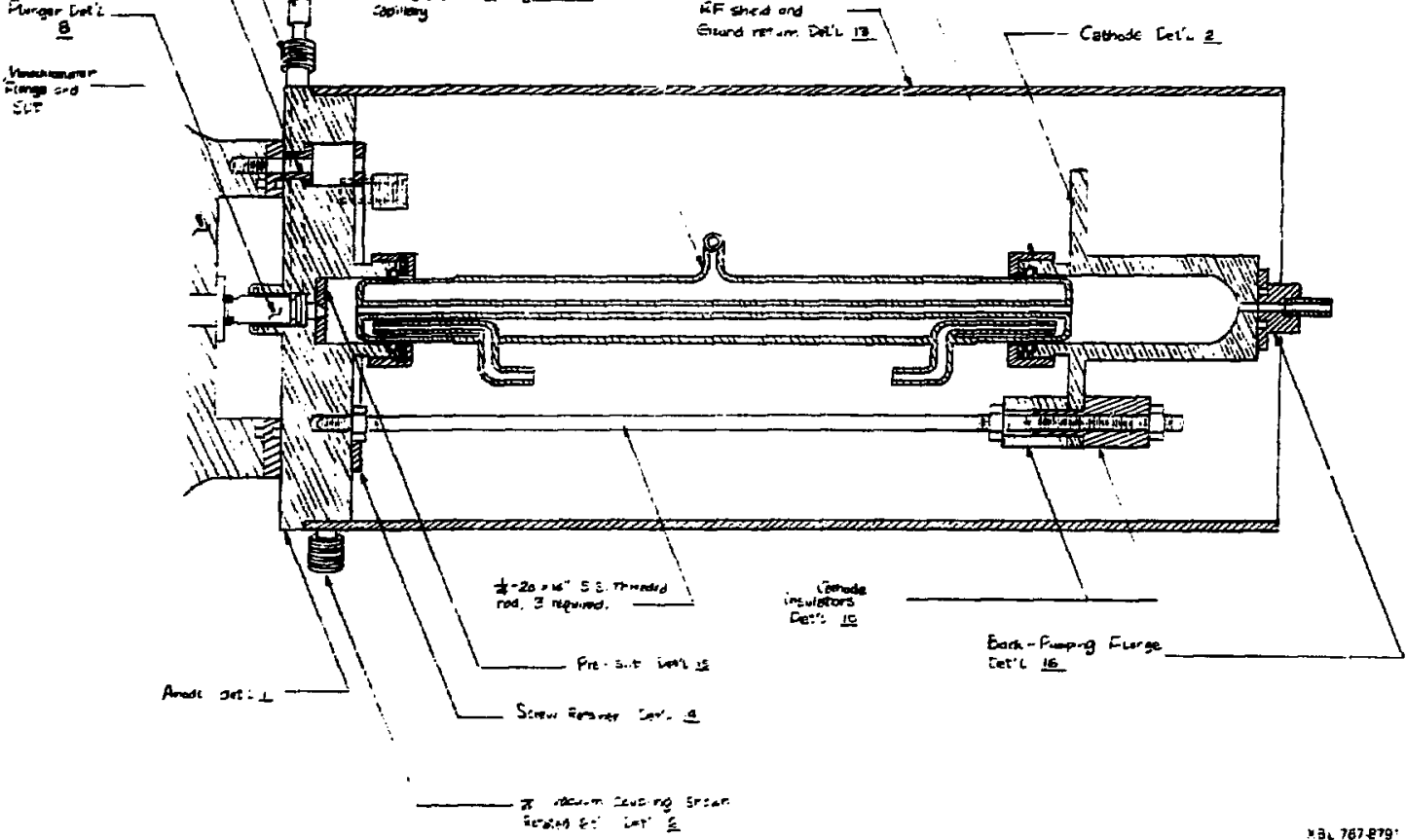

Fig. 7. Assembly of discharge light source. 


\section{Quadrupole Mass Spectrometer}

The assembly of the quadrupole mass spectrometer system is shown in Fig. 1. The whole detector chamber is pumped by a $220 \mathrm{\ell} / \mathrm{sec}$ (ULTEK) ion pump and a liquid nitrogen trap which is not shown in the drawing. A small manual operated gate valve is installed at the entrance of the quadrupole mass spectrometer system to isolate the detector chamber from the main chamber when the machine is not operating. During the operation of the apparatus, the detector is maintained at a pressure of $-5 \times 19^{-9}$ torr. The ions generated at the collision center efther by photon or electron impact are focused to the entrance of the quadrupole. After mass filtering, the ions are focused again, accelerated and then bombard an aluminum coated target which ejects two to seven electrons per ion, depending on the mass of the bombarding ions. These electrons are further accelerated on an aluminum coated thin plastic scintillator ${ }^{1}\left(0.0005^{\prime \prime}\right.$ thick) which is cemented on a quartz window with optical epoxy (which has approximately the same refractive index as the plastic scintillator (NE111). The light generated due to the impact of the electrons then further amplified by a photomultiplier (RCA 8850) which gives out a pulse which is then counted by a scaler. The electronic for the counting system is described in Section $F$. The assembly and electronic wiring for the lonizer and focusing ion optics is shown in Fig. 2. In order to eliminate the ion pump noise, a ground metal shield is placed around the scintillation detecting system. In addition, an optical baffle is installed at the pump port of the ion pump to prevent the soft $x$-rays from interfering with 
the scintillation detector. (Not shown in Fig. 1)

The general scintillation system can be understood by dividing the process into six consecutive events.

1. The absorption of $30 \mathrm{keV}$ electrons in the plastic scintillation system, resulting in excitation and fonization within it.

2. The conversion of energy dissipated in the scintillator to light energy through the luminescence process.

3. The transit of light photons to the photocathode of the photomultiplier tube.

4. The absorption of the photons at the cathode and the emission of the photoelectron.

5. The electron-multiplication process within the photomultiplier tube.

6. The analysis of the current pulse furnished by the photomultiplier tube through the use of the succeeding electronic equipment.

If $n_{e}$ is the number of photoelectrons released from the photocathode and $M$ is the multiplication factor of the tube, then the charge $q$ appearing at the output of the photomultiplier is

$$
\begin{aligned}
q & =\operatorname{Men}_{e} \\
n_{e} & =\left({ }_{n} E_{n} F_{n} C_{n p}\right)\left(T_{p} F_{p}\right)\left(S_{m} F_{c}\right)
\end{aligned}
$$

$E_{n}$ is the energy of the high energy electrons

$F_{n}$ is the fraction of total energy of bombarding electrons absorbed by the scintillator.

$C_{n p}$ is the efficiency of conversion from electron energy dissipated in 
the scintillator to light energy.

$T_{p}$ is the transparency of scintillator for scintillation which it produces.

$P_{p}$ is the fraction of light photons which reach photocathode if $T_{p}=1$.

$S_{m}$ is the sensitivity of photocathode at wavelength for maximum sensitivity in terms of photoelectrons per electron volt of light energy striking it.

$f$ is the figure of merit expressing the degree to which the spectral sensitivity of photocathode matches spectral distribution of scintillation.

$F_{c}$ is the fraction of photoelectrons collected by dynodes. Let $n$ to be the number of high energy electrons, then ${ }^{n}{ }_{n} F_{n} C_{n p}=$ the amount of light energy in the scintillation.

$T_{p} F_{p}=$ fraction of light which reaches the photocathodes. $S_{m f} F_{c}=$ number of photoelectrons reaching the first dynode of the multiplier per electron volt of light energy striking the photocathode.

The output pulses of the phototube are inductively coupled to the counting electronic system (fig. 5). The photomultiplier used in this apparatus has a quantum yield of $\sim 40 \%$ which corresponds a value of $S_{m}=0.1$, let us assume

$$
\begin{aligned}
& T_{p} F_{p}=0.5 \\
& F_{n} C_{n p}=0.03 \\
& S_{m f} F_{c}=0.05 \\
& n=6 \\
& M=5 \times 10^{6}
\end{aligned}
$$


The output pulse width is $-2 \times 10^{-9} \mathrm{sec}$.

With a $50 \Omega$ connector, the pulse height is

$$
\begin{aligned}
& V=I R=\left(\frac{q \text { coul }}{2 \times 10^{-9} \mathrm{sec}}\right)(50 \Omega) \\
& n_{e}=\left(6 \times 30 \times 10^{3} \times 0.03\right)(0.5)(0.05)=140 \\
& q=140 \times 1.9 \times 10^{-19} \times 5 \times 10^{6}=1.33 \times 10^{-10} \mathrm{coul} \\
& V=\frac{1.33 \times 10^{-10} \times 50}{2 \times 10^{-9}}=3 \mathrm{volt}
\end{aligned}
$$

In order to examine the detector, pulse height analysis has been carried out with the set up as shown in Fig. 3. The pulse height distribution with different cathode voltages and electron energies is shown in Fig. 4. To discriminate the single electron noise from the photocathode, the discriminating level only set at approximately 0.2 volt. 
$-164$

\section{REFERENCES}

1. N. R. Daly, The Rev. of Scientific Inst., 31, 264 (1960).

2. W. J. Price, "Nuclear Radiation Detection" McGraw Hill Book Company, 2nd Ed. (1964). 


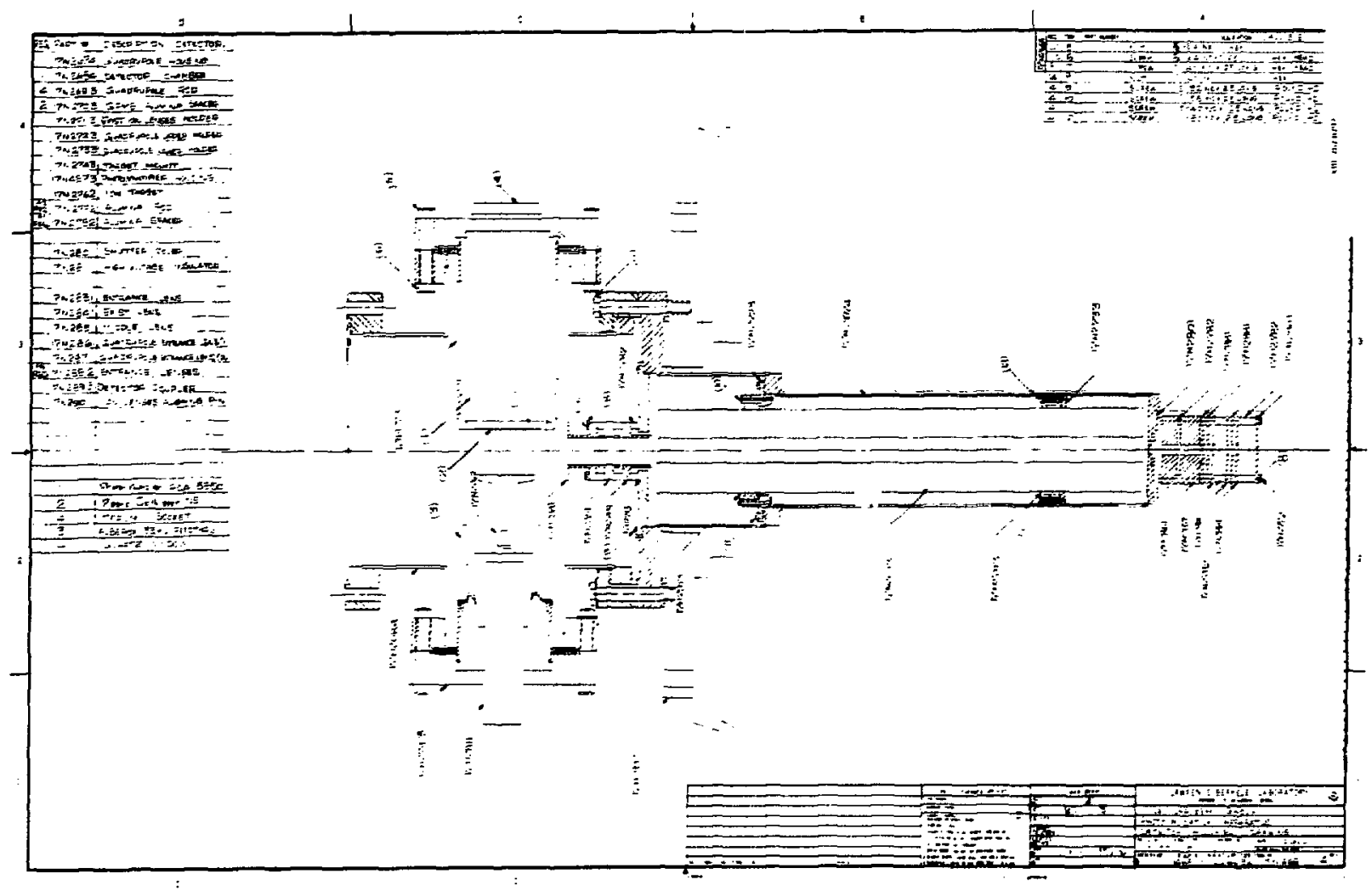

Fig. 1. Assembly of quadrupole mass spectrometer. 


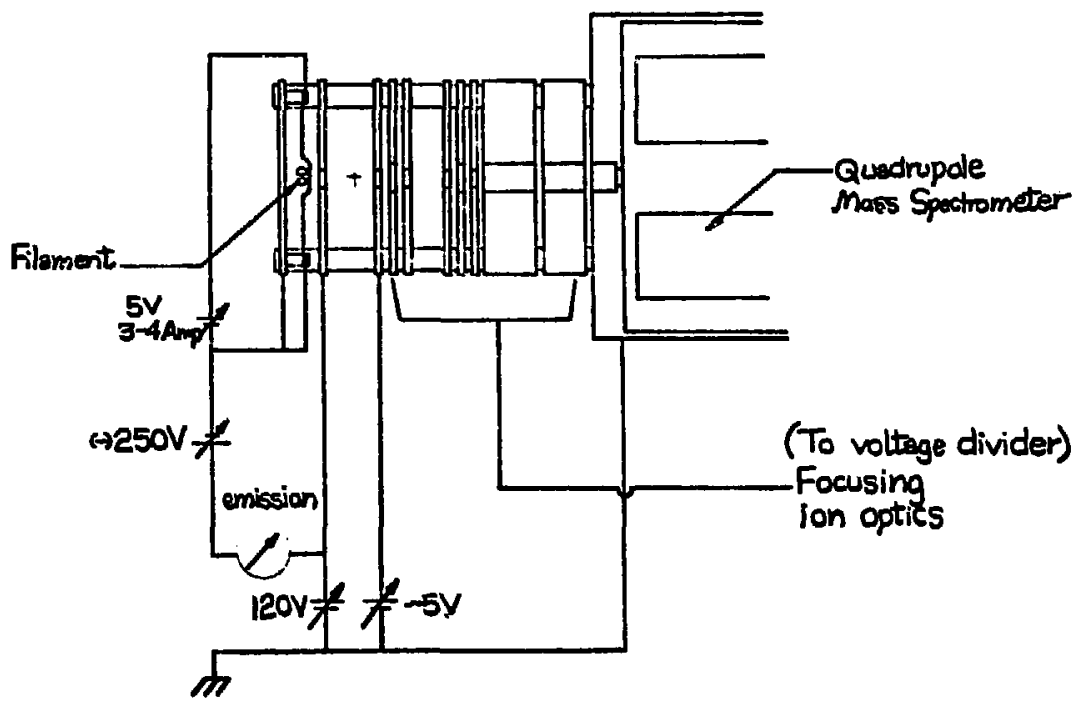

Fig. 2. Assembly of the ionizer and focusing elements 


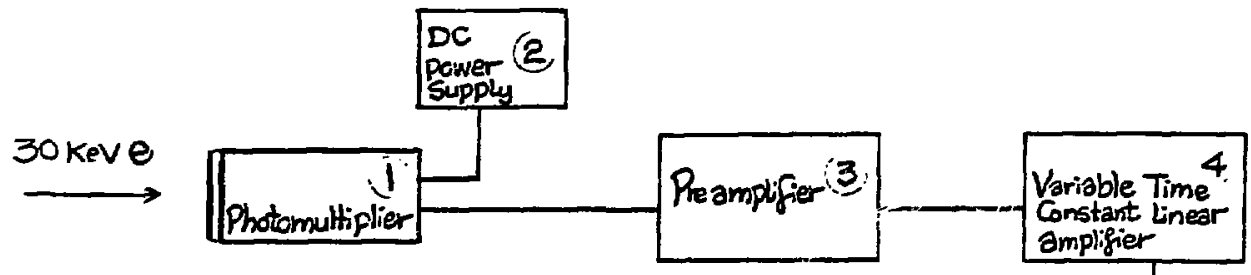

(1) RCA 2850 Photomultiplier

(2) Regulated high voltage power supply Model RE-3002EEW

(3) LBL $27 X 2257$ P7

(4) LBL $21 \times 1011$ P-1

(5) Packard Multichannel Analyzer Mode 116

(6) Mode H44562A Hewlett Packard

(7) 125? Terminator

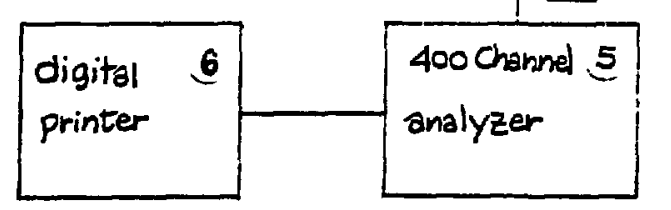

Fig. 3. Rulse Height Anaysis Mode 


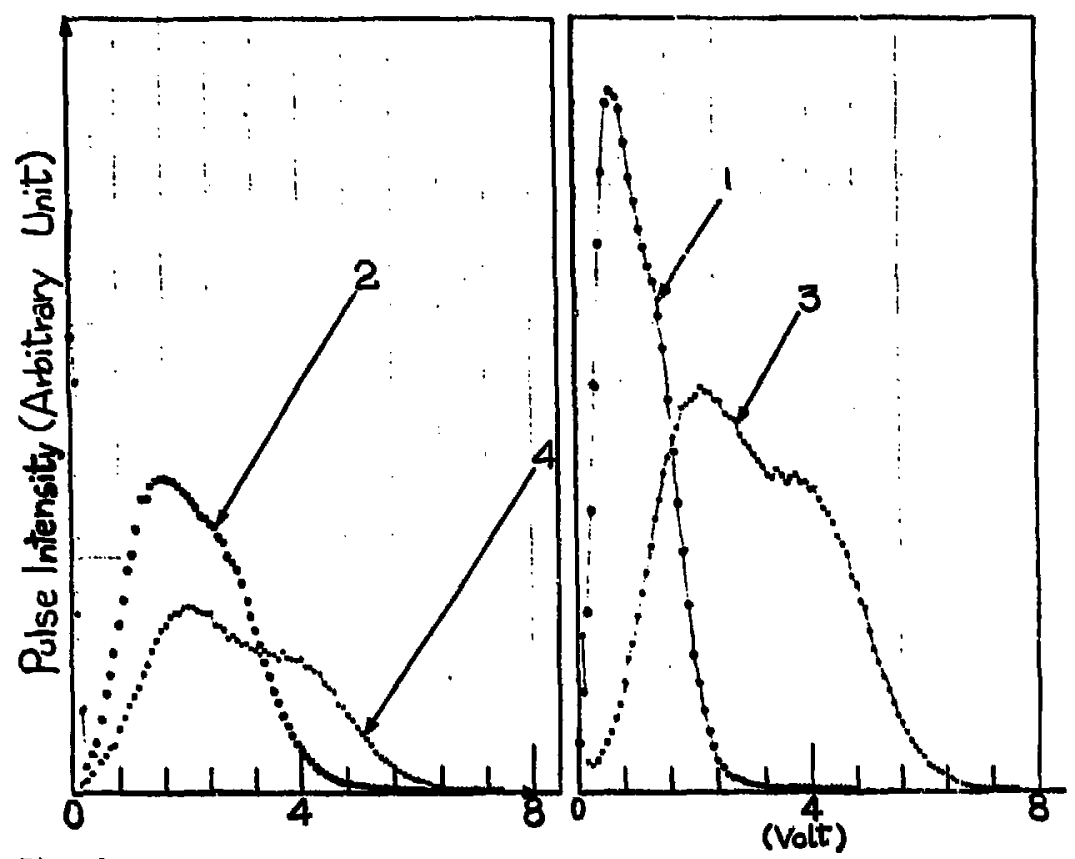

Fig. 4.

Amplifier at a gain $=4$

Curve 1: Phototube cathode voltage $=(-) 2150 \mathrm{~V}$, electron energy $=30 \mathrm{keV}$ Curve 2: Phototube cathode voltage $=(-) 2250 \mathrm{~V}$, electron energy $=25 \mathrm{keV}$ Curve 3: Phototube cathode voltage $=(-) 2250 \mathrm{~V}$, electron energy $=30 \mathrm{keV}$ 


\section{()$=$ Pin Numbers}

\section{PAINTED ON ELECTROSTATIC}

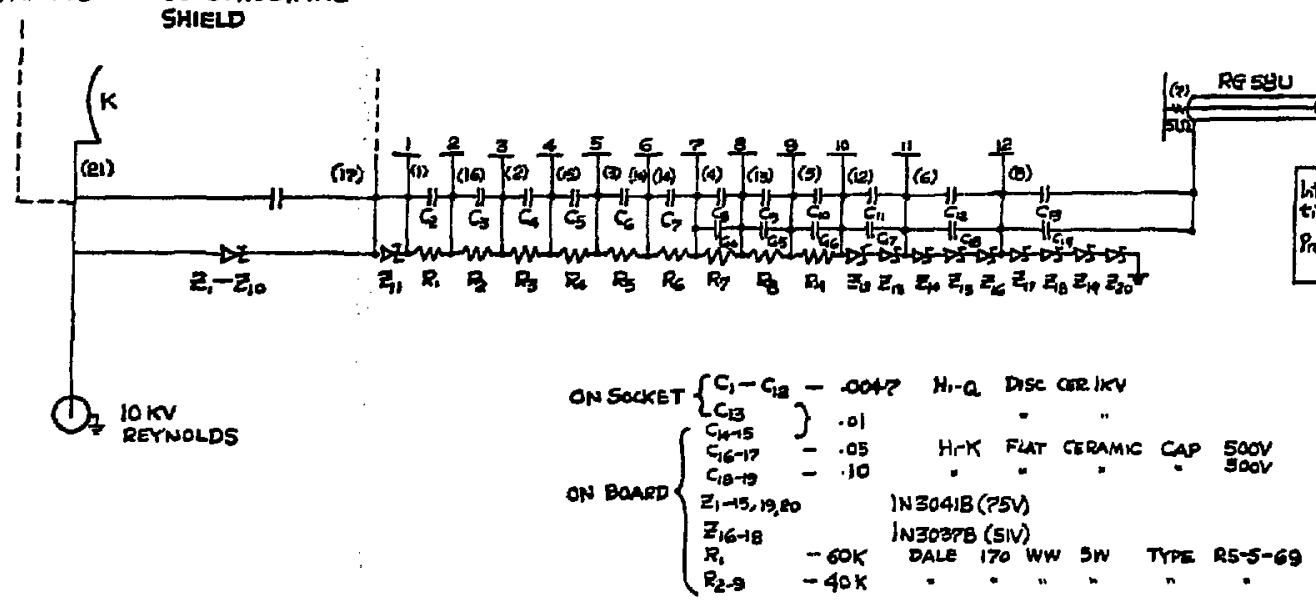

Fig. 5. RCA 8850 BASE WIRING (LBL 21X227 S-1) 
E. The Vacuum U1 traviolet Photon Detectors

The various modes of interaction between radiation and matter provide the underlying principles of all detectors. For vacuum UV radiation, these interactions involve the photoionization of gases, the ejection of photoelectrons form metals, chemical changes, photoconductivity, fluorescence, and thermal effects. Two types of VUV photon detectors are used in this research.

I. (a) Fluorescent Detector.

This technique essentially combines the use of a photonultiplier with a scintillator. The scintillating material used is sodium salicylate which has been found to be one of the materials that have the highest fluorescent efficiency in the range $400-2000 \AA$. The assembly diagran of the fluorescent detector is shown in Fig. 1. A fine crystalline layer of sodium salicylate is coated on a quartz window. The latter is cemented to an aluminum flange. The impingement of VUV photons produces a fluorescent emisston spectrum as shown in Fig. 2. ('ihe maximum intensity of fluorescence is located at $4200 \AA$ and coincides with the maximum sensitivity of a photomultiplier.) The fluorescent signal is thus amplified by the phototube and measured by a picoarmeter. The properties of sodium salicylate have been well studied. Figure 3 shows the relation between response and thickness of the sodium salicylate layer at $1200 \AA^{2}$ It can be seen that the efficiency rises rapidly to a maximum at $1 \mathrm{mg} / \mathrm{cm}^{2}$ then falls off very slowly as the thickness increases. The relative quantum efficiency of sodium salicylate between 200 and $1000 \AA$ is depicted in Fig. $4 i^{3}$ and Fig. $5^{4}$ shows the aging efficiency of coating. 
It was found that the fluorescent spectrum of sodium salicylate was independent of the exciting wavelength between 275 and $2537 \AA$. $^{5}$ The fluorescent decay time of sodium salicylate appears to lie between 7 and 12 nsec. 6,7

I. (b) Nickel Photoelectric Detector.

The photoelectric yields of most metals have been measured from 2 - 1500A. 8,9 It was found that for the spectral region from $473 \AA$ to $1000 \AA$, the photoelectric yield of a heat treated nickel cathode is nearly constant. Using a nickel sheet as the cathode in a simple diode arrangement, the total photoelectric emission current $I(\lambda)$ measured is related to the incident photon flux by the following relation:

$$
\operatorname{Ni}(\lambda)=\left(I(\lambda) \times 6.24 \times 10^{18}\right) / \gamma(\lambda) \text { photon } / \text { sec }
$$

where $\gamma(\lambda)$ is photoelectric yield of nickel.

The mechanical drawing for the construction of the nickel photoelectric detector is shown in Fig. 6. 
$-172-$

REFERENCES

1. F. S. Johnson, K. Watanabe and R. Tousey, J. Opt. Soc. Am., 4l, 702 (1951).

2. M. Seya and F. Masuda, Sci. Light, 12, 9 (1963).

3. J.A.R. Samson, unpublished data (1965).

4. J.A.R. Samson, J. Opt. Soc. Am., 54, 6 (1964).

5. K. J. Nygaard, Brit. J. Appl. Phys., 15, 597 (1964).

6. K. J. Nygaard, private communication (March 1965).

7. G. K. Herb and W. J. Van Sciver, Rev. Sci. Inst., 36, 1650 (1965).

8. W. C. Walker, N. Wainfoin and G. L. Weissler, J. Appl. Phys., 26, 1366 (1955).

9. H. Hinteregger and K. Watanabe, J. Opt. Soc Am., 43, 604 (1952). 


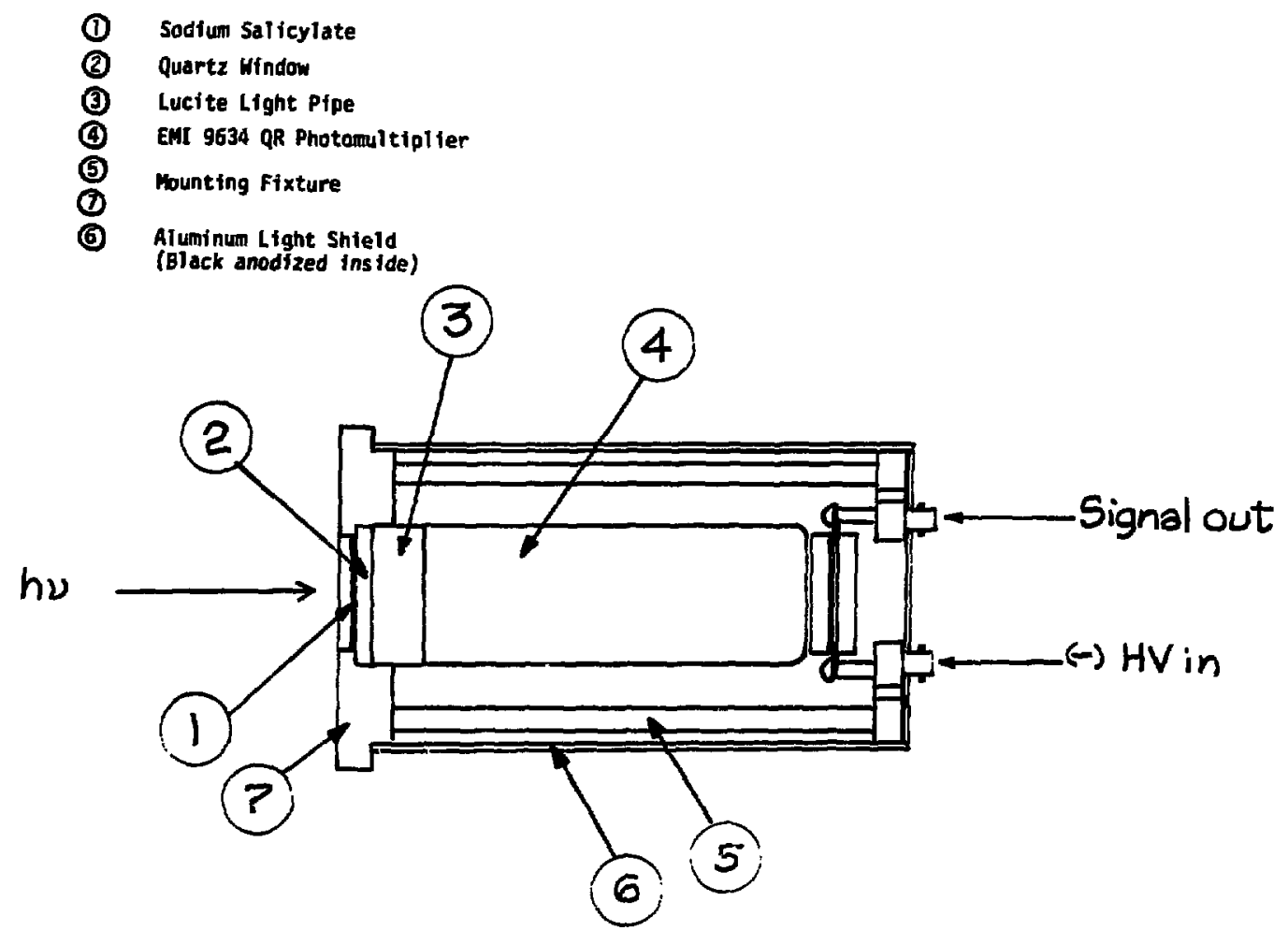

Fig. 1. Assembly of the fluorescent detector 


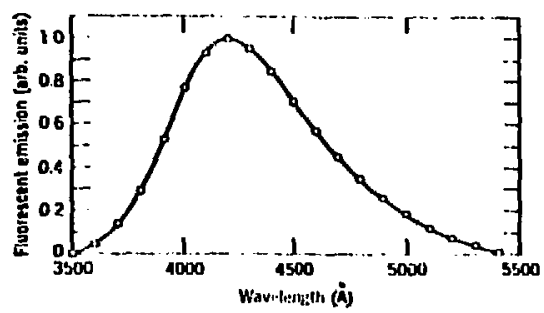

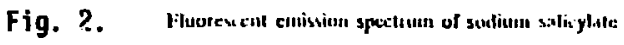

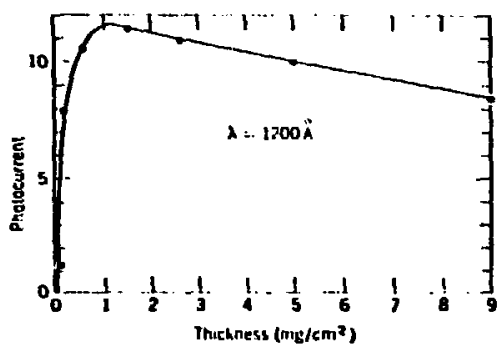

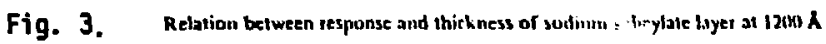

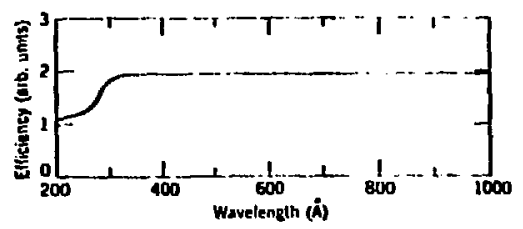

Fig. 4. Relative quantun efficiency of sodium salicylitte between 200 and $1000 \mathrm{~A}$.

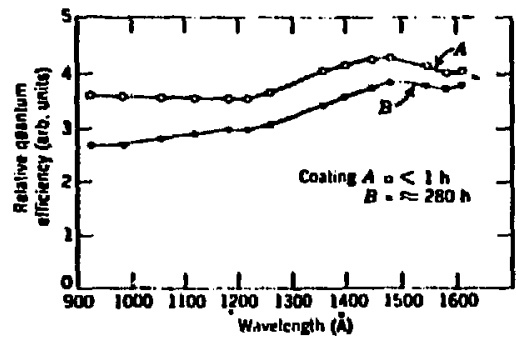

Fig. 5. Relative quanlum efficiency of adium salicylate between 900 and $1600 \mathrm{~A}$. The yield was measured for Iwo differeul coatings relative in a thermnpile, Costing $A$ is fess than one hour old while cosating $A$ is approkintuately $2 \mathrm{HO}$ howrs old 


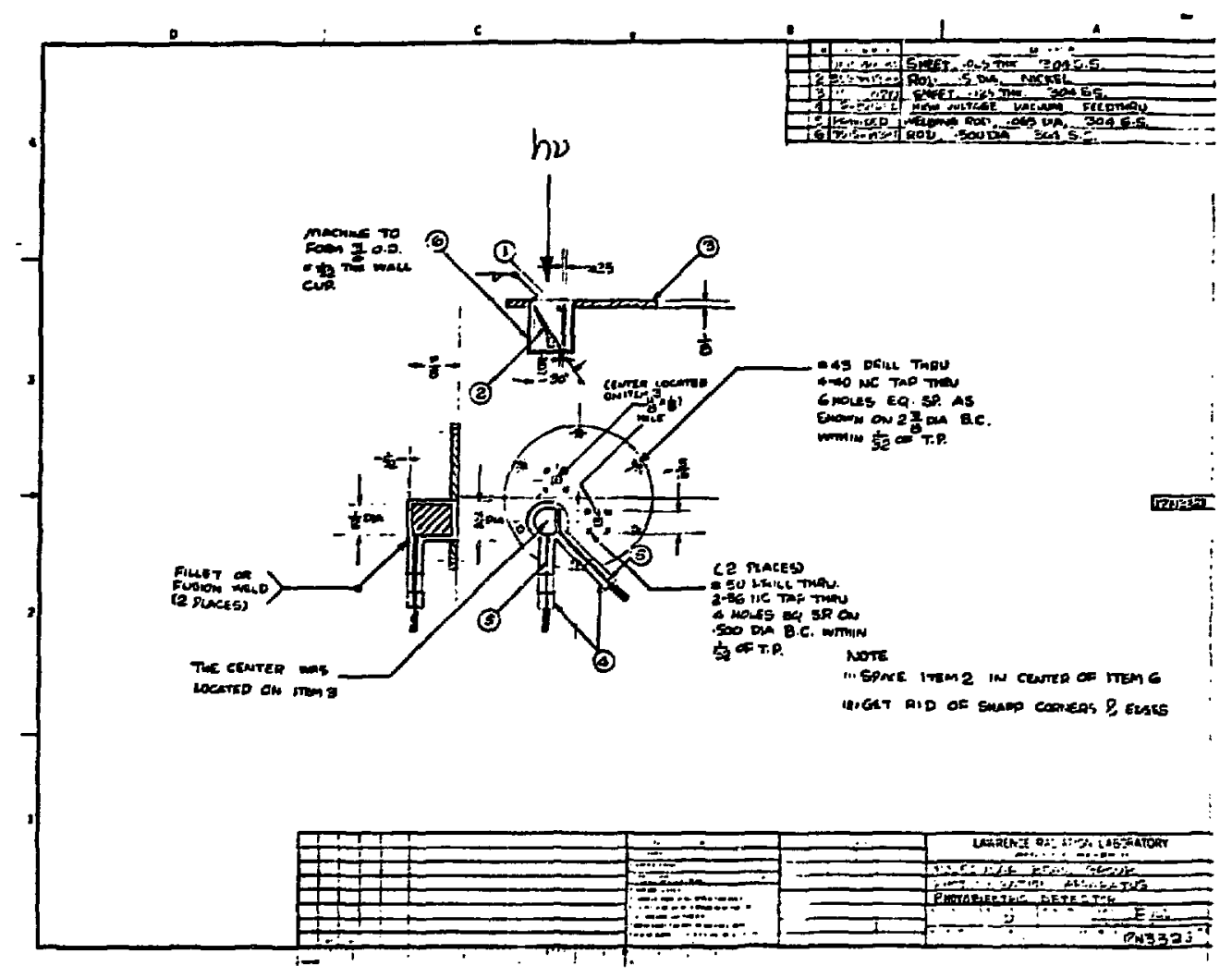

Fig. 6. Photoelectric detector 


\section{F. Automatic Scan Control System for Photoionization Apparatus}

The electronic block diagram for the photoionization apparatus is shown in Fig. 1. During the photoionization experiment the ion intensity and the photon intensity at a corresponding wavelength have to be monitored simultaneously. The fon and photon detection in the manual mode have been described in the experimental part of chapter 3 . This section describes the operation of the photoionization apparatus in an automatic mode. The scan control timing diagram for the photoionization apparatus is shown in Fig. 2. The system is initiated by pressing the start control on the timer. A puise (time out or gate 3) with the width corresponding to the present time on the timer will enable the third scaler to count the pulses generated by the voltage to frequency converter (this measures the photon intensity in number of counts). The rising edge of this pulse also initiates gates 1 and 2 which gate scaler 1 and 2 respectively. These two gates are generated by the $150 \mathrm{~Hz}$ chopper as mentioned in the experimental part of Chapter 3. The signal of scaler 1 corresponds to the photoion intensity when the chopper is opened and scaler 2 gives the background fon signal when the chopper is closed. The falling edge of the time out pulse (which synchronizes with the falling edge of the last pulse of gate 2) then energizes the oscillator of the printer to print out the signals of the three scalers. Simultaneously, after the printing, the falling edge of the last printing pulse triggers two pulses to reset the timer and scalers as well as the automatic clock pulses (3ps width) to step the stepping motor upward or downward to a preset number of steps 
(80 step for $1 \AA$ increment). The rising edge of the run count pulse advances the counter in the control unit from display 1 to 2 (i.e. the first run is complete) and generates a restart puise to start the timer for the second run. The system will continue until it has completed the number of runs as preset in the control unit.

The photon signal as measured by the picoammeter is also recorded by a strip chart recorder. This gives a secondary record of the photon intensity. The analog output $(0-1.5 \mathrm{~V})$ is converted to digital output by a voltage to frequency converter as mentioned above. In order to preserve the relative intensity of the photon, the voltage to frequency converter has to have a linear response to the voltage output from the picoammeter. Thus the linearity of the $V / f$ is examined and plotted in Fig. 3 which shows a relation

$$
f\left(K H_{2}\right)=59004 V+57
$$

where $V$ is the input voltage in volt and $f$ is the output frequency in KHz. 


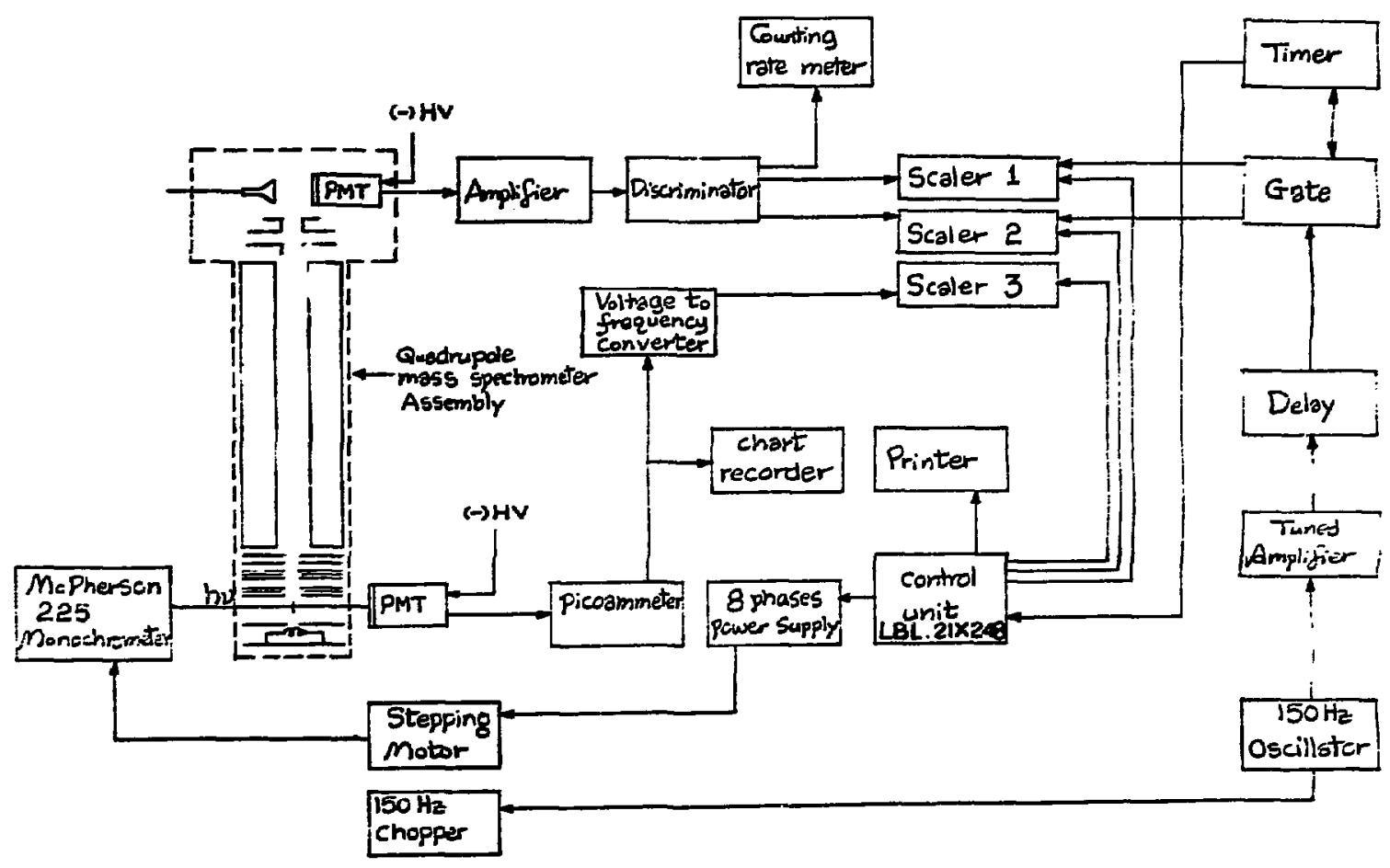

Fig. 1. Electronic block diagram of the photoionization apparatus 


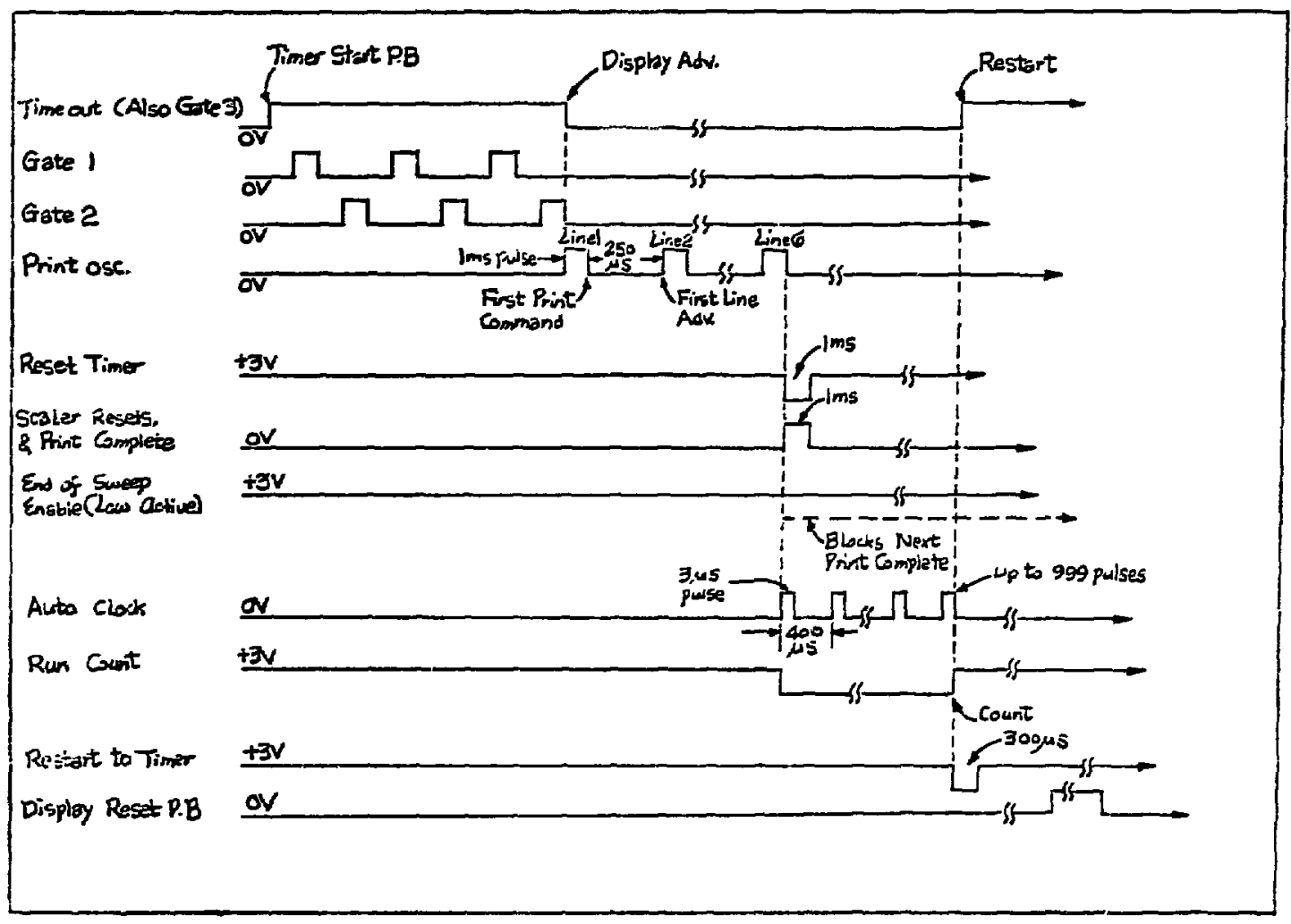

Fig. 2. SCAN CONTROL TIMING DIAGRAM 


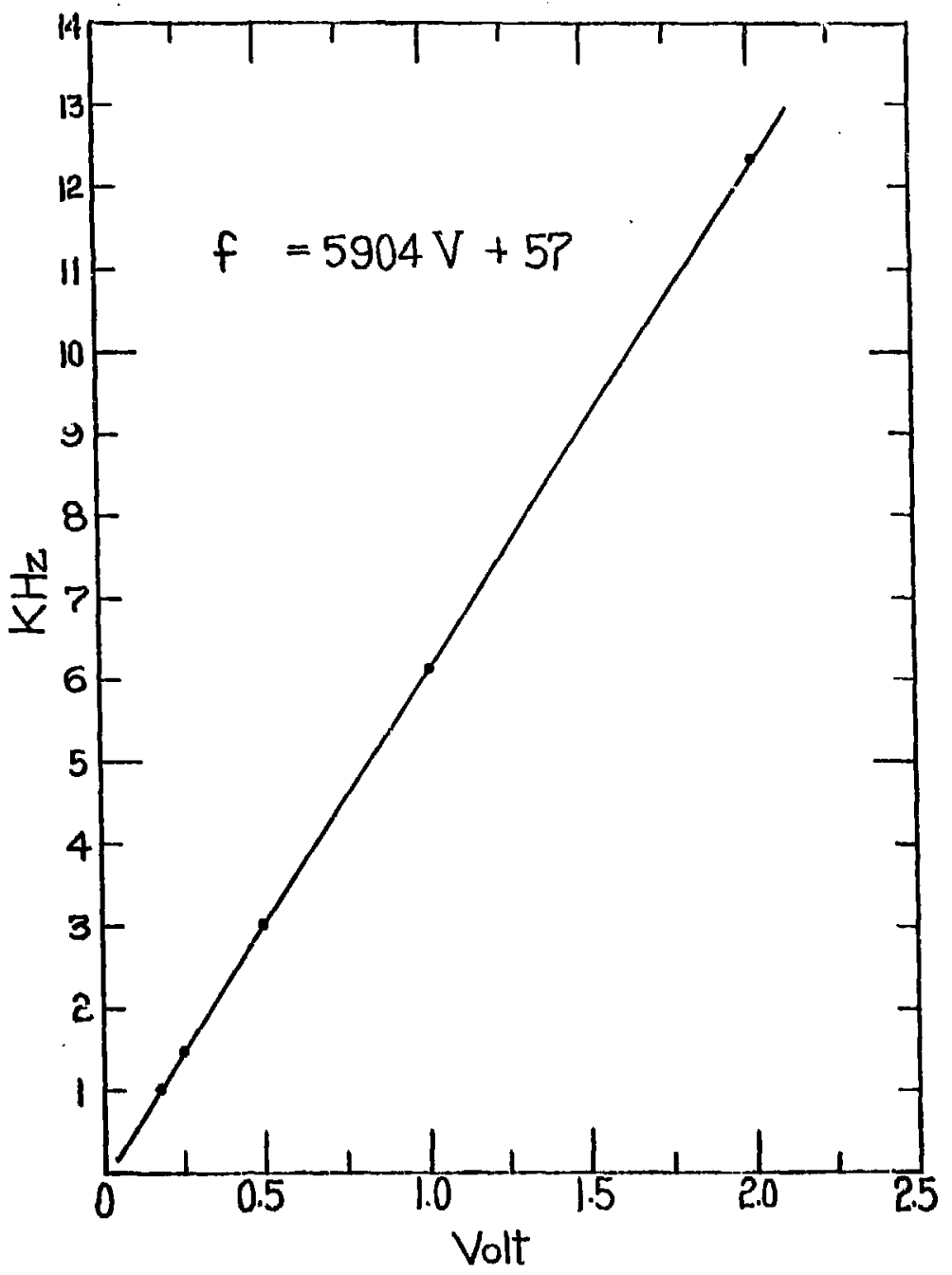

Fig. 6. Linearity plot of the voltage to frequency converter 